THE EFFECT OF LEARNING, FORGETTING, FATIGUE, AND RECOVERY ON THE PERFORMANCE OF DUAL-RESOURCE CONSTRAINED (DRC) SYSTEMS

\author{
by \\ Zahra Sadeghigivi \\ Bachelor of Science in Aerospace Engineering, Amirkabir University of Technology \\ (Tehran Polytechnic), Tehran, Iran, 1999 \\ Master of Science in Aerospace Engineering, Sharif University of Technology, Tehran, \\ Iran, 2002
}

\begin{abstract}
A dissertation
presented to Ryerson University
\end{abstract}

in partial fulfillment of the

requirements for the degree of

Doctor of Philosophy

in the program of

Mechanical and Industrial Engineering

Toronto, Ontario, Canada, 2014

(C) Zahra Sadeghigivi, 2014 


\section{AUTHOR'S DECLARATION}

\section{AUTHOR'S DECLARATION FOR ELECTRONIC SUBMISSION OF A DISSERTATION \\ I hereby declare that I am the sole author of this dissertation. This is a true copy of the dissertation, including any required final revisions, as accepted by my examiners.}

I authorize Ryerson University to lend this dissertation to other institutions or individuals for the purpose of scholarly research.

I further authorize Ryerson University to reproduce this dissertation by photocopying or by other means, in total or in part, at the request of other institutions or individuals for the purpose of scholarly research.

I understand that my dissertation may be made electronically available to the public. 


\title{
The effects of learning, forgetting, fatigue, and recovery on the performance of Dual-Resource Constrained (DRC) systems
}

\author{
Zahra Sadeghigivi \\ Doctor of Philosophy \\ Mechanical and Industrial Engineering \\ Ryerson University, Toronto, 2014
}

\begin{abstract}
Dual-Resource Constrained (DRC) systems consist of two resources: workers and machines (stations). DRCs have become common in manufacturing and service firms that emphasise flexibility, where workers perform different tasks. Although having a flexible workforce is beneficial, it comes at a cost. When workers alternate between different jobs the productivity of the system is affected. On one hand the system becomes more responsive to changes (internal/external), and on the other hand worker productivity and system throughput deteriorate because of the loss of knowledge and workers' fatigue. This subjects workers to conflicting phenomena. When workers are performing a task they are learning but also accumulating fatigue, which may result in error or injury. When transferred to another task, or on a break, workers may forget what they have learnt but at the same time recover from fatigue, either fully
\end{abstract}


or partially. In particular, forgetting and fatigue are interesting to be considered as they directly affect the quality of products.

This research investigates the effects of workers' learning-forgetting and fatigue-recovery on DRC systems. First, it modifies a known learning-forgetting model by accounting for fatigue and recovery. Second, it assumes that the quality of a production process may deteriorate and generate defective items that require rework. Third, a human error model is developed that considers human learning-forgetting and fatigue-recovery in producing defective items. Fourth, a comprehensive model is developed that integrates learning, forgetting, fatigue, and recovery into a DRC system with quality consideration. This model is investigated for different transfer and flexibility policies. Numerical results provide insights and guidelines that may help operations managers with decisions on how to improve a system's performance and throughput, while considering worker welfare. Results indicate that it is important to consider workers capabilities and limitations when designing manufacturing systems. They also suggest that ignoring human restrictions and abilities results in unrealistic production planning and erroneous cost estimation. 


\section{Acknowledgements}

I would like to express my sincere gratitude to my supervisor and mentor, Professor Mohamad Y. Jaber, who made my doctorate journey possible by his moral and financial support. His scholarly guidance, vision, patience, and persuasion encouraged me to accomplish this tough job while allowing me to work in my own way. He has been a continuous source of immense intelligence, wisdom, and tenacious dedication to scholarly life that motivated me and helped me in every step. He was always available for advice and help by giving me valuable feedback and guidelines to proceed. Sir, you are one of the most honorable persons I have ever met in my life.

My sincere thanks go also to my co-supervisor Professor W. Patrick Neumann, who provided me a pleasant and responsive environment for my research and always helped me with his encouragement, thoughtful comments, and friendly attitude.

I would also like to thank my committee members, Professor S. Zolfaghari and Dr. S. Taghipour, who helped me improve my research by their precious advice and comments. My special thanks go to Professor Zolfaghari who has continuously supported me in every challenge I pursued despite his very busy schedule. Furthermore, I would like to express my appreciation to the members of the Mechanical and Industrial Engineering (MIE) Department at Ryerson University specially Professor J. Friedman, the chair of the department, and Professor Z. Saghir, and also

the administrative staff, notably, Lynn Reynolds, Karen Fajardo, Dahlia Younan, and Grace He. You all made my PhD study a pleasant experience at the MIE department by your kindliness, support, and help. Lynn Reynolds, thank you for being such an inspiring and thoughtful leader. 
In addition, I am thankful to my fellow lab mates Ahmad Sobhani, Michael Greig, Shane Dixon, Judy Village, and Pete Dodé. Thank you for welcoming me at the lab as a friend and helping me in developing ideas for my research. I should also thank my colleagues Ehab Bazan and Fatemeh Firouzi for their help whenever I asked for it. My special thanks go to Professor Linda Rose for her knowledgeable advice and her interest in my research.

I take this opportunity to thank Dr. H. Ghaemi, Professor Z. Fawaz, and Professor J. Lassaline at the Aerospace Department at Ryerson University for their support and expansive help in various occasions. I am particularly thankful to Professor Abdul-Kader at University of Windsor for reading my thesis with a critical eye and offering precious comments.

I am also grateful to the Dean of the Faculty of Engineering, Architecture, and Science, Yeates School of Graduate Studies at Ryerson University, the Natural Science and Engineering Research Council of Canada (NSERC), and Social Sciences and Humanities Research Council (SSHRC) for providing the financial support for my research.

Finally, I would not have contemplated this road without my husband, Nima, and my smart and athlete daughter, Solar, who have been a continuous source of help and support. I sincerely thank them for their patience, understanding, and ideas, especially in the past four years that made my doctorate voyage possible and successful. My gratitude also goes to my mother and to the soul of my father who planted the seed of science, education, and language in me. And lastly, I am grateful to my sister Fateme, for being with me throughout every journey in my life.

\section{Zahra Sadeghigivi}

Toronto, Canada

September 2014 


\section{Table of Contents}

Author's Declaration .................................................................................................................

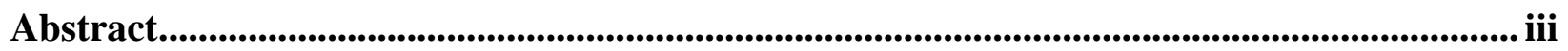

Acknowledgements ................................................................................................................................................... v

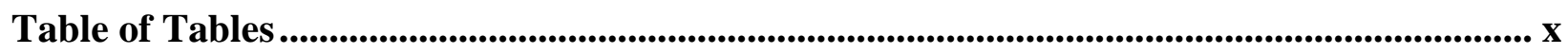

Table of Figures ........................................................................................................................................................ xiv

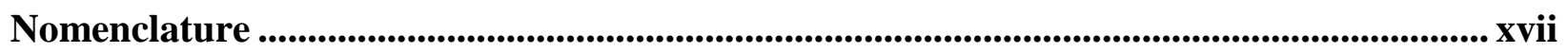

Acronyms ............................................................................................................................................................ xix

1. Introduction ................................................................................................................................................ 1

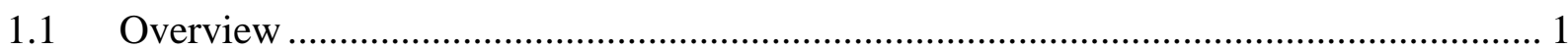

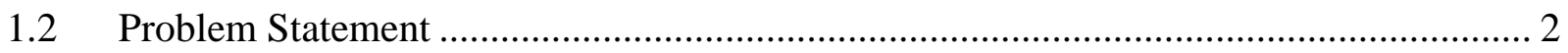

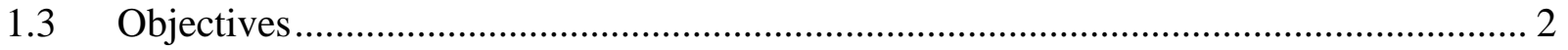

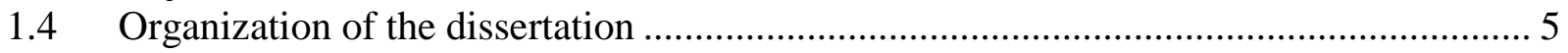

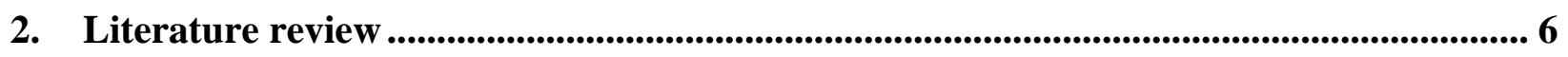

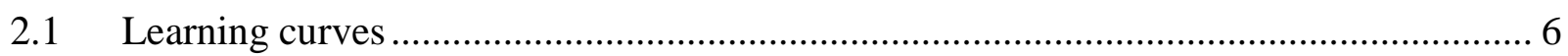

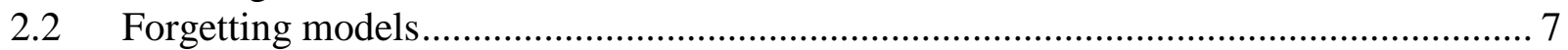

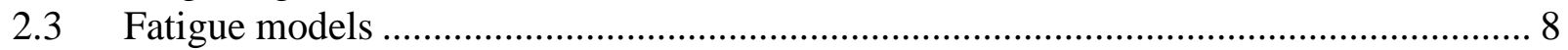

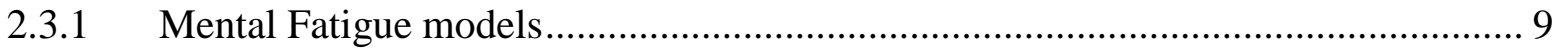

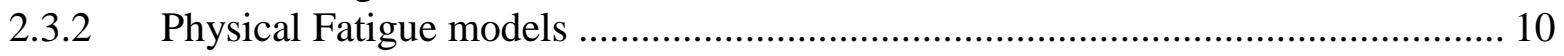

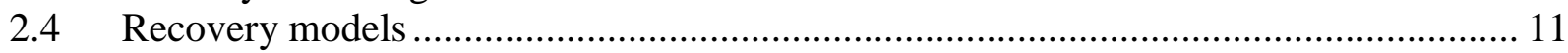

2.5 DRC systems with learning and forgetting ………................................................. 12

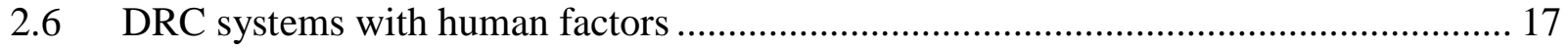

2.7 DRC systems with fatigue and recovery .............................................................. 19

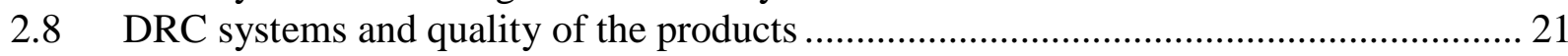

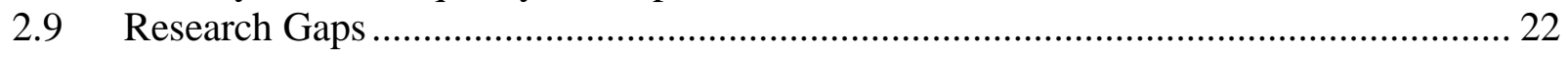

3. Framework and Methodology .................................................................................................. 24

3.1 Phase 1: Incorporating fatigue-recovery into the learning-forgetting model ................. 24

3.2 Phase 2: Incorporating forgetting into the quality learning curve ………………….... 25

3.3 Phase 3: Investigating human error with learning-forgetting and fatigue-recovery ...... 26

3.4 Phase 4: Investigating flexibility and transfer policies in a DRC system with some

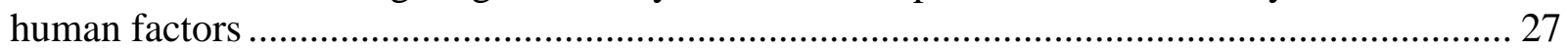

4. Phase 1: Incorporating fatigue-recovery into the learning-forgetting model................... 29 


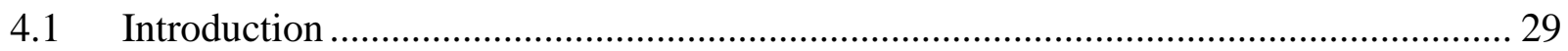

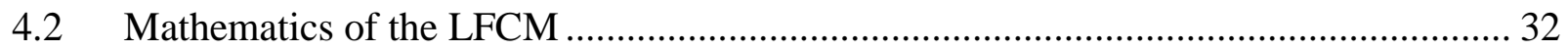

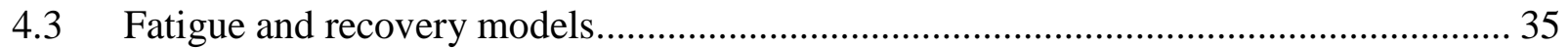

4.4 The Learning Forgetting Fatigue Recovery Model (LFFRM) ..................................... 39

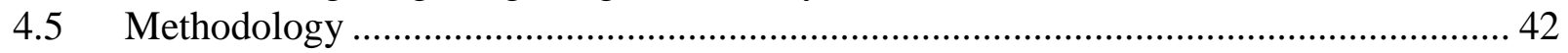

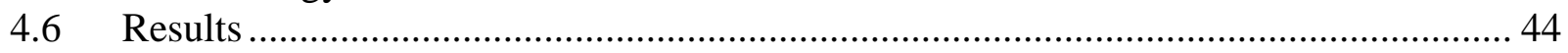

4.6.1 What effect does the learning index have on the production process? .................. 44

4.6.2 What effects does the number of batches have on the production process? ........... 46

4.6.3 What effects does the fatigue index have on the production process? ................... 47

4.6.4 What effects does the recovery index have on the production process?................. 49

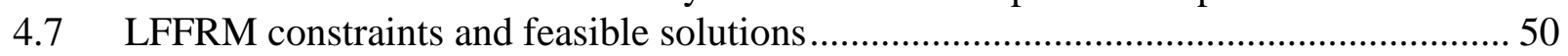

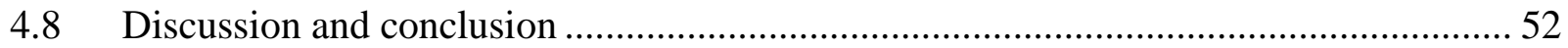

\section{Phase 2: Incorporating forgetting into the quality learning curve, Quality Learning} Forgetting Curve (QLFC) .................................................................................................56

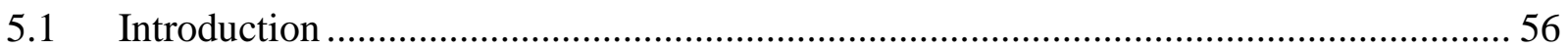

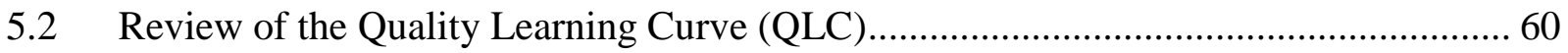

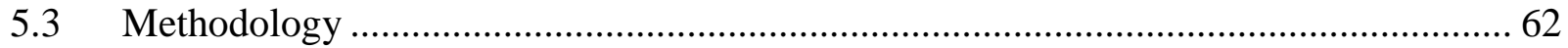

5.3.1 Learning in production and no learning in rework $(\varepsilon=0.0)$................................ 62

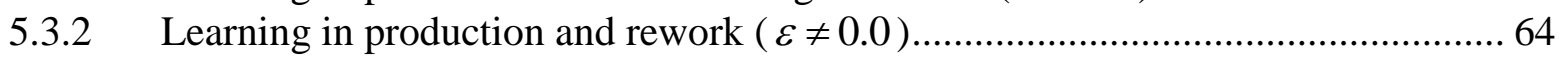

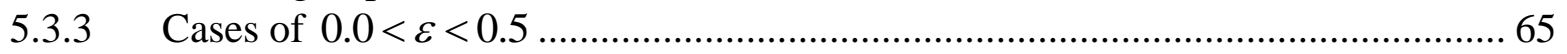

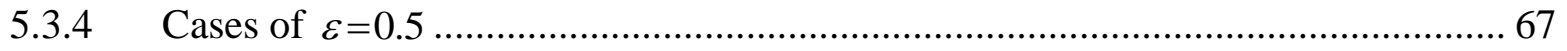

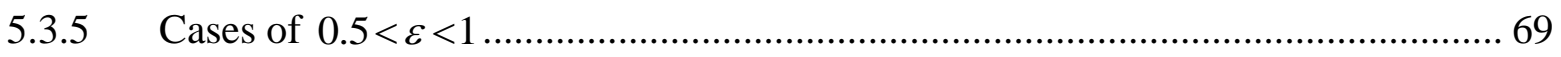

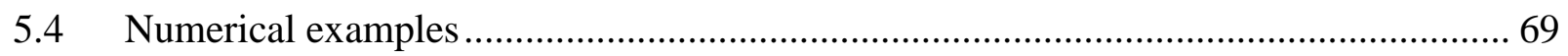

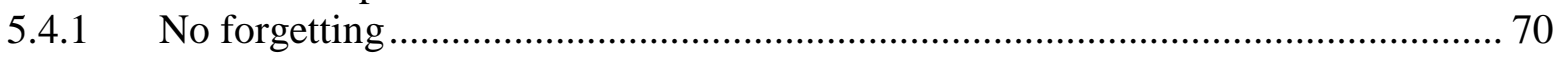

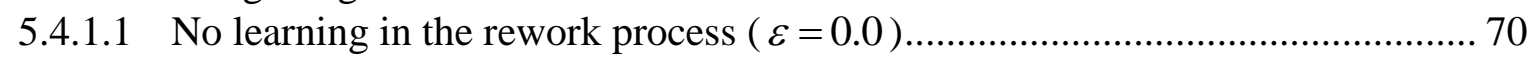

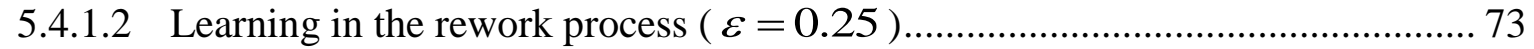

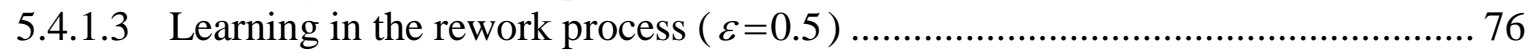

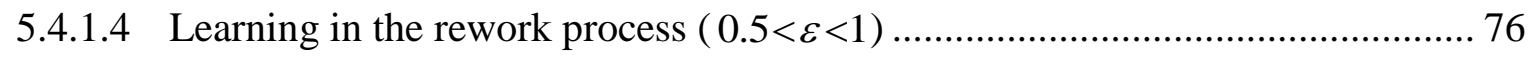

5.4.2 Forgetting Cases.............................................................................................. 77

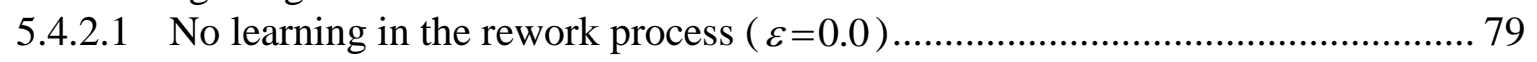

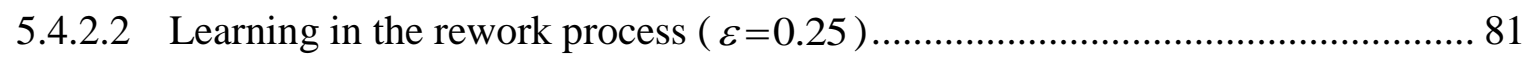

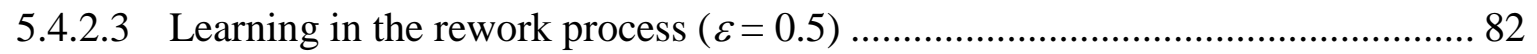

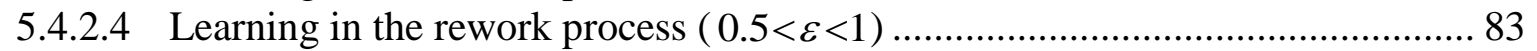

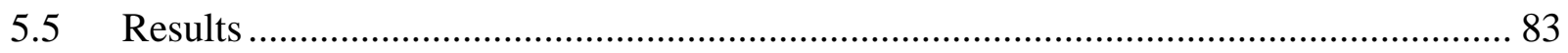

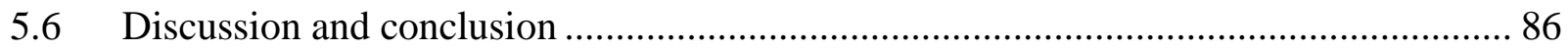

6. Phase 3: Investigating human error with learning-forgetting and fatigue-recovery .... 88

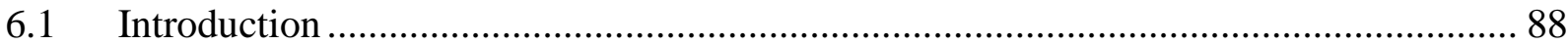

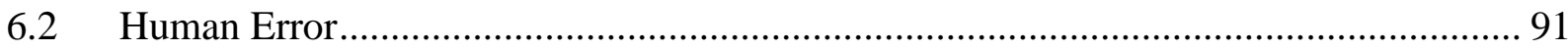

6.2.1 Human error and learning-forgetting ................................................................ 92

6.2.2 Human error and fatigue-recovery ......................................................................... 94 


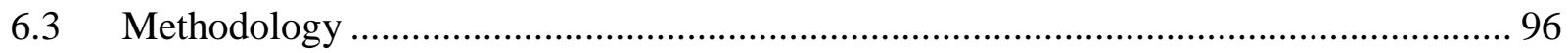

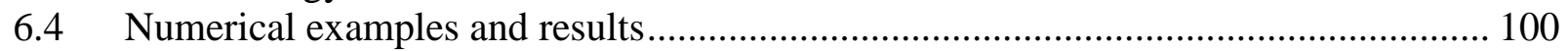

6.5 Discussion and conclusion ................................................................................. 113

7. Phase 4: Investigating flexibility and transfer policies in a DRC system with some human factors.................................................................................................................................. 116

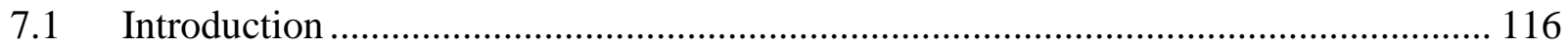

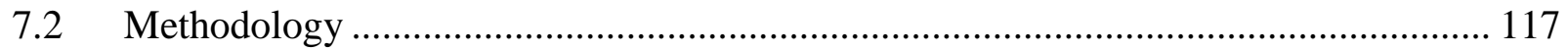

7.2.1 Performance Measure ………………..................................................... 119

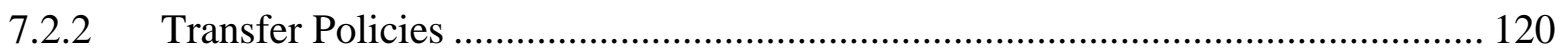

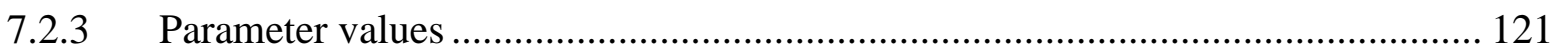

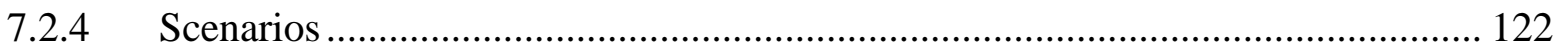

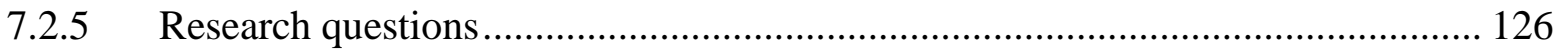

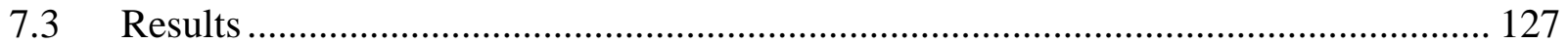

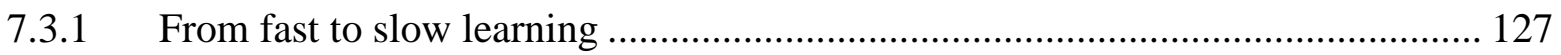

7.3.1.1 From fast to slow learning and hard to easy work ......................................... 127

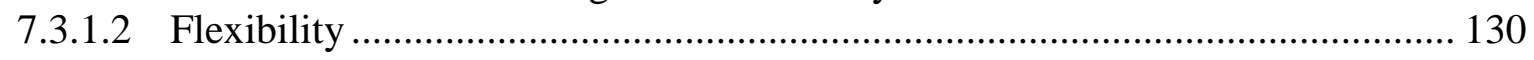

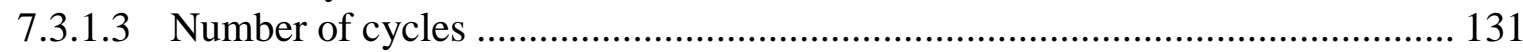

7.3.1.4 From fast to slow learning and easy to hard work ......................................... 132

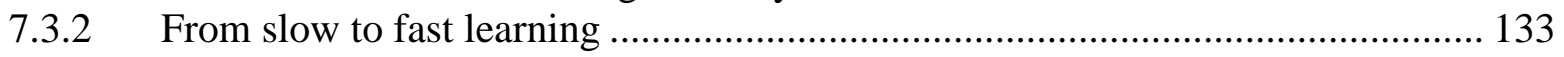

7.3.2.1 From slow to fast learning and hard to easy work .......................................... 133

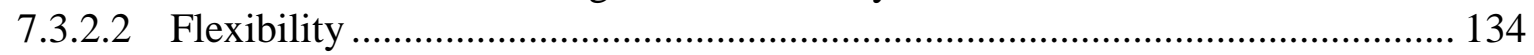

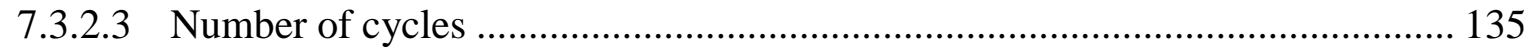

7.3.2.4 From slow to fast learning and easy to hard work …………......................... 135

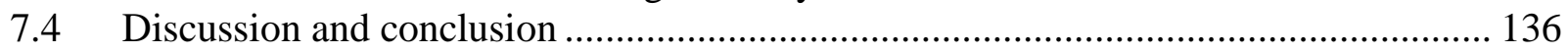

8. Contributions and Future Directions .................................................................................. 139

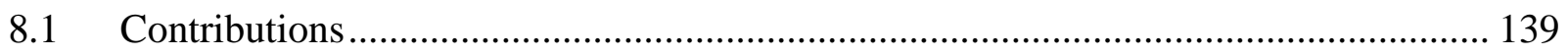

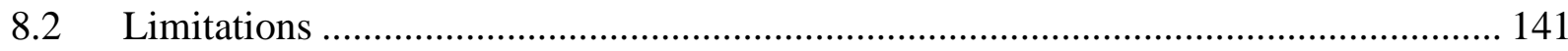

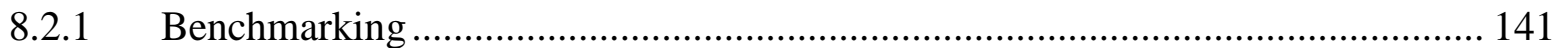

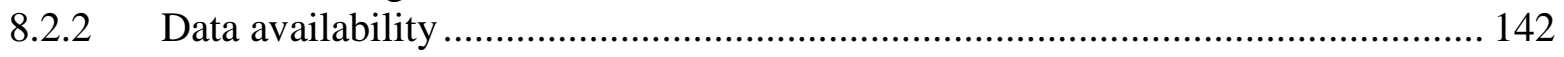

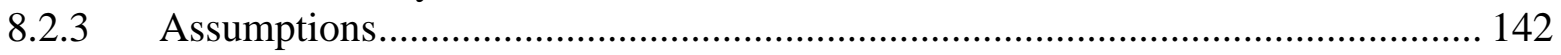

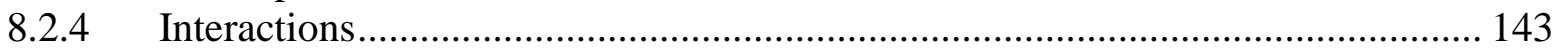

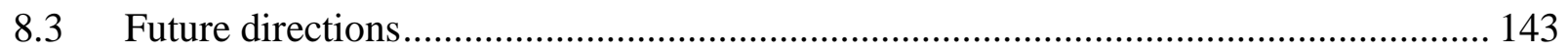

9. Summary and Conclusions ................................................................................................................. 145

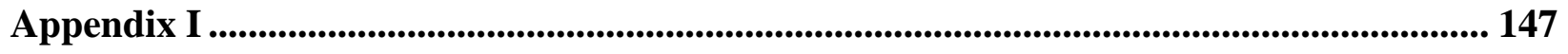

References...................................................................................................................................................... 148 


\section{Table of Tables}

Table 2.1. The contribution of the proposed research to the industrial engineering literature..... 23

Table 4.1. Typical learning rates adapted from Crawford (1944), higher rates represent slower

learning. 33

Table 4.2. The effect of learning index on the process outputs when $\lambda=0.03$ and $\mu=0.05$. 45

Table 4.3. The effect of the number of batches on the production process when $b=0.322$, $\lambda=0.03$, and $\mu=0.05$. 47

Table 4.4. The effect of fatigue index on the production process when $b=0.322$ and $\mu=0.05 .48$

Table 4.5. The effect of recovery index on the production process when $b=0.322$ and $\lambda=0.03$.

Table 5.1. Parameters' initial values. 69

Table 5.2. Optimum values of the lot size, $x^{*}$, with learning in production, no learning in rework, and no forgetting. 72

Table 5.3. Performance on the learning curve for the last unit produced as per Table 5.2. 72

Table 5.4. Optimum values of the lot size, $x^{*}$, with learning in production and rework, and no forgetting. 74

Table 5.5. Performance on the learning curve for the last unit produced as per Table 5.4. 74 
Table 5.6. Plateau values of the composite learning curve when there is no forgetting in the learning and rework processes, for all $b>0$, and $\varepsilon=0.5$. 76

Table 5.7. Optimum values of the lot size, $x^{*}$, with learning and forgetting in production and no learning in rework. 80

Table 5.8. Performance on the learning curve for the last unit produced as per Table 5.7. 80

Table 6.1. Example of categorization of parameters affecting human error ${ }^{*}$ as per ElMaraghy et al. (2008). 92

Table 6.2. Work schedule in a shift with four working cycles adding up to 455 minutes 100

Table 6.3. Parameters' initial values. 100

Table 6.4. Average human error probability for different learning indices, fatigue and recovery indices are set at medium values. 105

Table 6.5. Average human error probability for different fatigue accumulation indices, learning and recovery indices are set at medium values. 105

Table 6.6. Average human error probability for different recovery indices, learning and fatigue accumulation indices are set at medium values. 105

Table 6.7. The results of the base case (production of 600 units over a 455 minutes work shift). 105

Table 6.8. Weight setting for maximum throughput, problem statement. 106 
Table 6.9. Weight setting for maximum throughput, solution. 106

Table 6.10. Schedule optimization for maximum throughput, problem statement. 109

Table 6.11. Schedule optimization for maximum throughput, solution.

Table 6.12. Schedule optimization for minimum fatigue, problem statement. 109

Table 6.13. Schedule optimization for minimum fatigue, solution. 109

Table 6.14. Effect of additional break time on optimal schedules, problem statement. 110

Table 6.15. Effect of additional break time on optimal schedules, solution 110

Table 6.16. Effect of additional break time on optimal schedules, lunch break > 30 minutes, problem statement.

Table 6.17. Effect of additional break time on optimal schedules, lunch break > 30 minutes, solution 111

Table 6.18. The best case and worst case scenarios representing the spectrum of human error probability of the presented model. 113

Table 7.1. Values of the input parameters used in the numerical study. 122

Table 7.2. Performance measure investigation when learning and fatigue indices decrease from the first to the last working station (Learning: F=Fast, M=Moderate, S=Slow; Workload: $\mathrm{H}=\mathrm{Hard}, \mathrm{M}=$ Moderate, $\mathrm{E}=$ Easy). 128 
Table 7.3. Performance measure investigation when learning index decreases and fatigue index increases from the first to the last working station (Learning: F=Fast, M=Moderate, S=Slow; Workload: $\mathrm{H}=$ Hard, $\mathrm{M}=$ Moderate, $\mathrm{E}=$ Easy).

Table 7.4. Performance measure investigation when learning increases and fatigue decreases from the first to the last working station (Learning: F=Fast, M=Moderate, S=Slow; Workload: $\mathrm{H}=\mathrm{Hard}, \mathrm{M}=$ Moderate, $\mathrm{E}=$ Easy).

Table 7.5. Performance measure investigation when learning and fatigue indices increase from the first to the last working station (Learning: F=Fast, M=Moderate, S=Slow; Workload: $\mathrm{H}=$ Hard, $\mathrm{M}=$ Moderate, $\mathrm{E}=$ Easy). 136 


\section{Table of Figures}

Figure 4.1. An illustration of the behaviour of the learning-forgetting process over repeated work-rest cycles. 34

Figure 4.2. An illustration of the behaviour of the fatigue and recovery process over repeated work-rest cycles. 38

Figure 4.3. The behaviour of the systems optimal performance for different learning indices.... 45

Figure 4.4. The behaviour of the fatigue curve for different $\lambda$ values representing different levels of task difficulty. Production volume declines as the task difficulty increases. 48

Figure 5.1. Cycle $i$ consists of production and rework processes. The experiences $u(i)$ and $v(i)$ are transferred to the current cycle from previous runs. 78

Figure 5.2. The effect of restorations on the average unit time of the process when $b=0.25$ and $\varepsilon=0.0$. A single lot for $(i=1)$ is restored for $n$ times, $n=1, \ldots, 5$. 84

Figure 5.3. The effect of splitting a larger lot into smaller sublots with and without forgetting. The optimum lot size decreases with more lots. The forgetting effect prevents the lot size to decrease as per Table 5.2 and Table 5.7.

Figure 5.4. The effect of splitting a larger lot into sublots with and without forgetting. The plateau effects prevents the time per unit to decrease due to forgetting as per Table 5.3 and Table 
Figure 6.1. The learning error utility function over the working shift with small rises over breaks. 101

Figure 6.2. The fatigue error utility function over the working shift with declines over breaks. 101

Figure 6.3. Human error probability (HEP) with learning-forgetting and fatigue-recovery over the working shift, downward phases are the breaks. 103

Figure 7.1. This figure demonstrates 64 different scenarios created to address the research questions. $b \downarrow(\uparrow)$, indicates that learning is the fastest (slowest) at first and slowest (fastest) at the last workstation. $\lambda \downarrow(\uparrow)$ indicates that the work is hardest (easiest) at the first and easiest (hardest) at the last workstation. The Flex number, identifies the number of workstations. Each scenario is repeated in 15 or 25 cycles with the assigned workers that can be 1,2 , or 3 . The Table \# indicates where results are presented. 125

Figure 7.2. System performance $(\mathrm{Z})$ versus working cycles for 3 workstations and up to 3 workers with Flex $=3$ during 15 working cycles, learning and fatigue indices decrease from the first to the last working station. The inset provides a closer look of the performance of 2 workers declining after the $14^{\text {th }}$ and 3 workers declining after the $10^{\text {th }}$ cycle $($ WRK $=$ Worker $) \ldots \ldots \ldots \ldots . . . .128$

Figure 7.3. Performance indicator $Z_{1}$ increases asymptotically from zero over 15 working cycles for 3 workstations and up to 3 workers with Flex $=3$. Learning and fatigue indices decrease from the first to the last working station (WRK= Worker). 
Figure 7.4. Performance indicator $Z_{2}$ decreases from one over 15 working cycles for 3 workstations and up to 3 workers with Flex $=3$. Learning and fatigue indices decrease from the first to the last working station (WRK= Worker).

Figure 7.5. System performance (Z) versus working cycles for 4 workstations and up to 3 workers with Flex=4 during 15 working cycles, learning and fatigue indices decrease from the first to the last working station. The inset provides a closer look of the performance of 2 workers declining after the $11^{\text {th }}$ and 3 workers declining after the $8^{\text {th }}$ cycle (WRK= Worker). 130

Figure 7.6. System performance (Z) versus working cycles for 3 workstations and up to 3 workers with Flex=3 during 25 working cycles, learning and fatigue indices decrease from the first to the last working station. The inset provides a closer look of the performance of 2 workers declining after the $14^{\text {th }}$ and 3 workers declining after the $10^{\text {th }}$ cycle (WRK= Worker).

Figure 7.7. System performance $(Z)$ versus working cycles for 3 workstations and up to 3 workers with Flex=3 during 15 working cycles, learning index decreases and fatigue index increases from the first to the last working station. The inset provides a closer look of the performance of 2 workers declining after the $13^{\text {th }}$ and 3 workers declining after the $10^{\text {th }}$ cycle (WRK= Worker). 


\section{Nomenclature}

$i \quad$ A subscript identifying the lot/batch number or a production run

$b \quad$ Learning index, defining the rate of learning, $0 \leq b<1$

$\varepsilon \quad$ Learning index in the rework process, $0 \leq \varepsilon<1$

$f \quad$ Forgetting index, defining the rate of forgetting, $0 \leq f<1$

$\lambda \quad$ Fatigue index, specifying fatigue accumulation rate

$\mu \quad$ Recovery index, specifying recovery rate

$x_{i} \quad$ Number of repetitions (units produced) in batch $i$

$X \quad$ Cumulative number of produced items

$u_{i}$

Experience measured in number of units remembered at the beginning of lot/batch $i$ in the production process

$v_{i}$

Experienced measured in number of units remembered at the beginning of lot/batch $i$ in the rework process

$\varphi_{i} \quad$ Length of the process interruption period following lot/batch $i$

$\tau_{i} \quad$ Length of the rest break following lot/batch $i$

$S_{i}$ Number of items that would have been produced in lot/batch $i$ if no interruption occurred

$B \quad$ Time needed for total forgetting to occur

$T_{1} \quad$ Time to produce the first unit in the first production cycle

$r_{1} \quad$ Time to rework the first unit in the first rework cycle

$t_{x_{i}} \quad$ Time to produce $x_{i}$ number of items in batch $i$

MET Maximum Endurance Time at a given force level

MVC Maximum Voluntary Contraction; maximum force a muscle can generate voluntarily

$f_{\mathrm{MVC}} \quad$ Fraction of the maximum voluntary contraction $(M V C)$

$\delta \quad$ Fatigue alleviation factor which determines how much fatigue needs to be alleviated

$\theta$ Experience transfer factor, representing the transfer of experience between working cycles

$t\left(u_{i}+x_{i}\right) \quad$ Time to produce $x_{i}+u_{i}$ units continuously on the learning curve

$\alpha_{0}, \alpha \quad$ Model-specific parameters

$\beta_{0}, \beta \quad$ Model-specific parameters 


\begin{tabular}{ll}
$N$ & Total number of batches \\
$n$ & Number of process restoration to return the process to the "in-control" state \\
$d_{i}$ & Number of defective items in lot $i$ \\
$\rho$ & The probability of the process to go out of control and produces defective items \\
$\xi$ & The percentage of the production process time dedicated for the purpose of restoration \\
$N L S$ & Normalised learning score \\
$N F S$ & Normalised fatigue score \\
$T_{o p, i}$ & Operation time in cycle $i$ with learning effects \\
$T_{o p, 0}$ & Operation time in cycle $i$ without learning effects \\
$u_{l}$ & Learning induced error rate \\
$u_{f}$ & Fatigue induced error rate \\
$w_{l}$ & Learning weight \\
$w_{f}$ & Fatigue weight \\
$U$ & Human error rate \\
$H E P$ & Human error probability \\
$D E F$ & Defective units \\
$T P$ & Process throughput \\
$Z_{l}$ & Cost of the time criterion \\
$Z_{2}$ & Cost of the rework criterion \\
$Z$ & Performance measure \\
$F l e x$ & Number of workstations \\
\hline & Number of workers \\
\hline & Level of flexibility \\
&
\end{tabular}




\section{Acronyms}

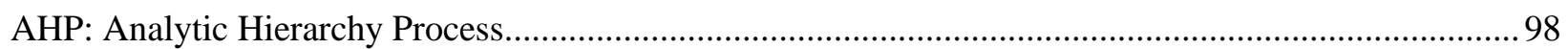

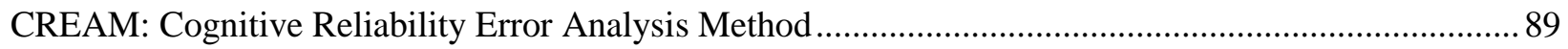

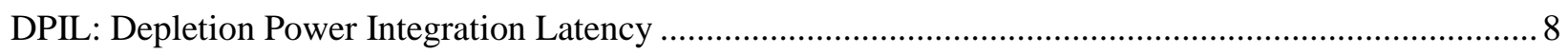

DPLFM: Dual-Phase Learning Forgetting Model ....................................................................... 14

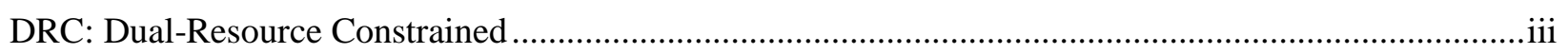

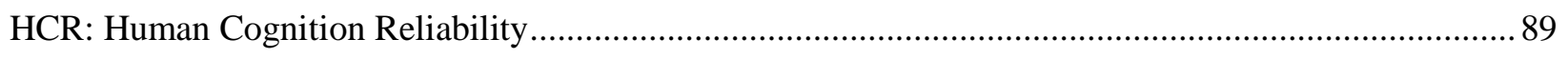

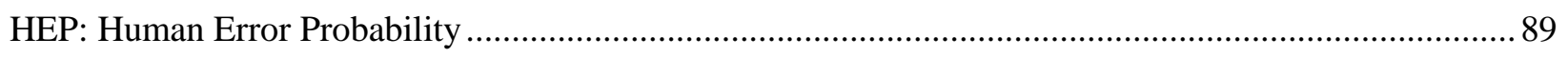

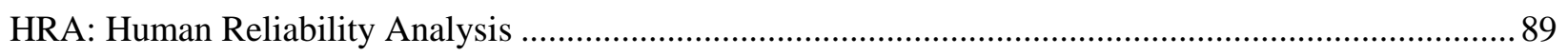

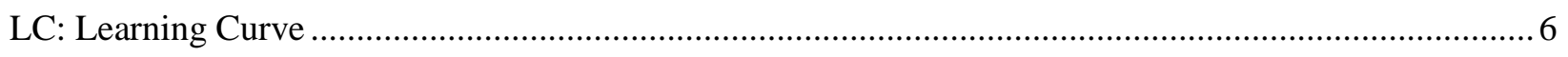

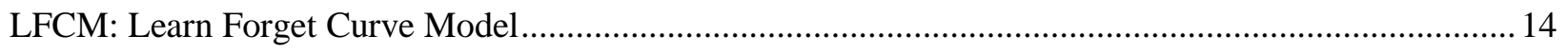

LFFRM: Learning Forgetting Fatigue Recovery Model................................................................ 24

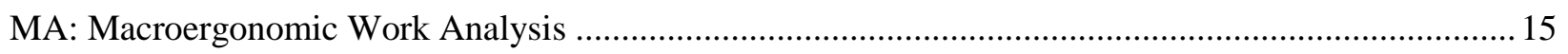

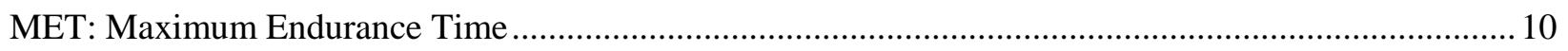

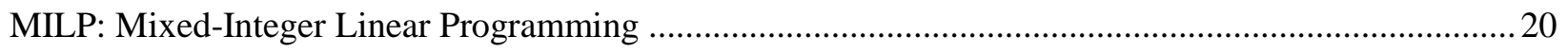

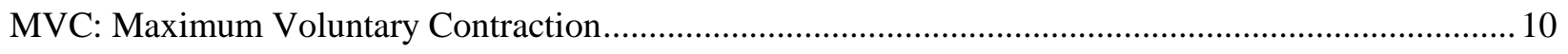

NIOSH: National Safety Institute of Occupational Safety and Health ................................................ 18

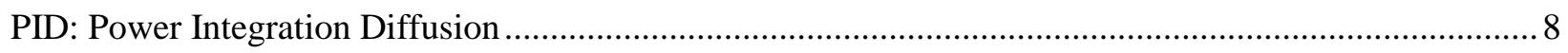

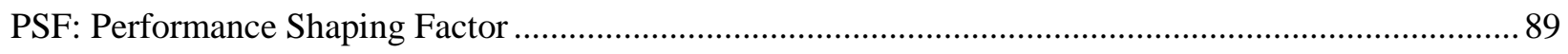

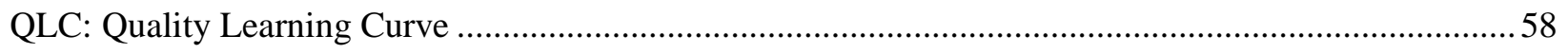

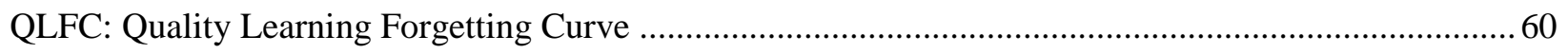

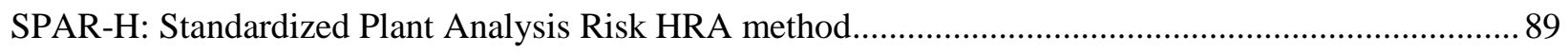

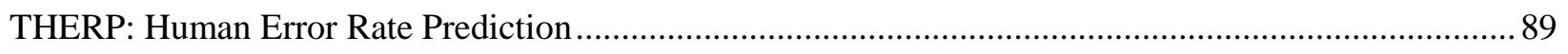

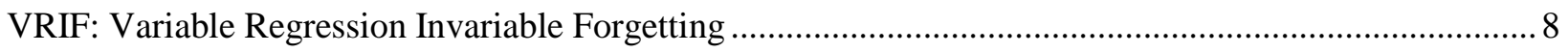




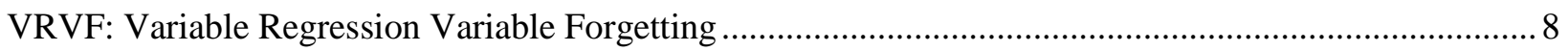

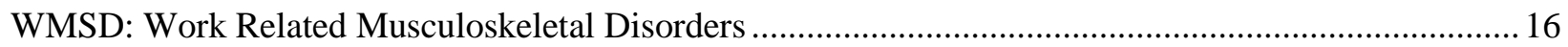




\section{Introduction}

\subsection{Overview}

Dual-Resource Constrained (DRC) systems are working environments where the number of workers are less than the number of machines or workstations (Zamiska et al., 2007). Workers in a DRC system are cross-trained to acquire several skills that increase their flexibility and allow them to perform a variety of tasks (Thannimalai et al., 2013). Cross-trained workers usually handle situations of unexpected orders and unbalanced workloads. A flexible workforce helps reduce lead times and improve customer service (Nembhard et al., 2002; Bokhorst \& Gaalman, 2009); however, having a fully cross-trained workforce is infeasible due to the cost of training and equipment (Gel et al., 2007; Robbins et al., 2007). In labour-intensive environments, workers accumulate experience (by learning through repetitions) which is partially lost (due to forgetting effects) when they are either on a break or transfer to perform a different task. Also, when performing a job, a worker accumulates fatigue, which is alleviated by rest breaks or moving to a physically less demanding job. The performance of a DRC system improves (impedes) through workers' learning (fatigue) and impedes (improves) because of forgetting (recovery). So, intermittent production cycles of learning/fatigue and forgetting/recovery have adverse effects on the system performance. While the performance of DRC systems may improve with the flexibility of workers (Jaber et al., 2003; Azizi et al., 2010; Jahandideh, 2012), alternating between different jobs or going on a break causes forgetting that impedes the performance and affects the quality. Learning and forgetting, fatigue and recovery, and quality in DRC systems have been studied separately, but, to the best of the author's knowledge, there is no study in the literature that captures the combined effect of these phenomena. The present thesis, 
contributes to the DRC system literature by presenting a production planning model that captures the aspects of human involvement and creates production schedules that are more realistic and applicable to such work environments.

\subsection{Problem Statement}

The analytical works that investigated the effects of learning and forgetting of workers on DRC systems are limited in number. Also, other human factors have not been given much attention in DRC systems, like workers' fatigue and recovery. Another human aspect that has not been considered in a DRC setting is human error.

Addressing above limitations will have a significant impact on production environments that require a flexible workforce. It will provide production managers with some guidelines regarding which factors to consider when improving the process and how these factors interplay and affect the performance and throughput of the systems they are managing.

The purpose of this research, therefore, is to develop an inclusive model for DRC systems that considers human abilities and limitations in terms of learning, forgetting, fatigue, and recovery. It also considers product quality that may be the result of the human behaviour indicated above.

\subsection{Objectives}

This research project is organised into four phases. In each phase, one or more research questions are posed and then addressed.

Phase 1 incorporates fatigue and recovery models into an existing learning and forgetting model. It aims to answer the following research questions: 
- How do fatigue and recovery constrain the performance of a system?

- What is the effect of interactions of the learning, forgetting, fatigue, and recovery behaviour of a worker on the process outcome?

- What effects do the learning, fatigue, and recovery parameters have on the production process?

- What effect does the number of batches have on the production process?

The results of this Phase of the research may help managers in addressing possible issues relating to workers' capabilities and limitations in a production environment. They also provide a basis for further research on the workforce scheduling and process improvement.

In Phase 2, a quality learning curve model is modified to account for the forgetting effect to answer the following research questions:

- How do learning and forgetting in the production process affect the lot size and batch policies?

- How do learning and forgetting in production and rework processes affect the lot size and batch policies?

- How do maintenance restorations affect the lot size and batch policies, and subsequently, process performance?

- How do maintenance restorations affect the standard time (learning plateau)?

The results of this Phase are useful in setting strategies for quality improvements, waste reduction, and for improving the standard time. 
In Phase 3, a Human Reliability Analysis model is developed that captures the interactions of learning, forgetting, fatigue, and recovery in generating human error and defective items. The following research questions are answered in this Phase:

- How do the learning-forgetting and fatigue-recovery behaviour of a worker incorporate in generation of defective items?

- How the learning, fatigue, and recovery parameters affect the human error rate?

- What work-rest schedules yield the highest process throughput or the lowest worker fatigue in the absence or presence of breaks?

The results of this Phase are useful to find the optimized schedules for increasing a system's throughput or improving a worker's welfare.

In Phase 4, the previous models are integrated into a DRC system with worker flexibility and transfer policies. Phase 4 of this research is the final phase of this research and accounts for learning-forgetting, fatigue-recovery, and process and product quality. The following research questions are answered in this Phase:

- How do accounting for learning-forgetting, fatigue-recovery, and product quality affect the performance of a DRC system?

- Is there an optimal flexibility level?

- Is there an optimal worker transfer policy?

The results of this Phase indicate that considering the human aspects in DRC systems generates more realistic policies than what currently are presented in the literature. 


\subsection{Organization of the dissertation}

The rest of this thesis is organized as follows. In Section 2, a literature review of DRC systems is presented and the existing research gaps are identified. Section 3 provides the framework and methodology of the research and states the research objectives. Sections 4, 5, 6, and 7 are representing Phases 1, 2, 3, and 4 of this research respectively. Section 8 provides contributions, limitations, and future directions of the thesis and Section 9 represents a summary of the previous Phases and concludes the thesis. 


\section{Literature review}

\subsection{Learning curves}

Learning is a phenomenon that has been studied in various industries from aircraft manufacturing (Wright, 1936; Benkard, 2000) to industrial processes (Argote \& Epple, 1990; Gulledge et al., 1990; Mitchell, 2000; Chatzimichali \& Tourassis, 2007) and service sectors (Reis, 1991; Vasdev et al., 2012). Waldman (2011) categorized the learning curve (LC) models into two groups: classical and inverted. In the classical learning curve, production cost (time) decreases with each repetition, while in the inverted LC, improvement is measured by the number of good quality outcomes. There is no consensus among researchers though whether improvement continues indefinitely (Wright, 1936), or it reaches a point beyond which no improvement occurs (see Jaber and Guiffrida, 2004 and 2008).

Factors such as task complexity, structure of training programs (Serel et al., 2003), worker's motivation (Azizi et al., 2010), and prior experience (Cherrington et al., 1987; Nembhard \& Uzumeri, 2000b) affect the learning behaviour of subjects. According to Anzanello and Fogliatto (2011) the most commonly used learning curve models are the Log-linear model (Wright, 1936), the S-shape model (Carr, 1946; Jordan, 1958), the Exponential model (Mazur \& Hastie, 1978), the Hyperbolic model (Gulliksen, 1934), and the Multivariate model (Gold, 1981). Among these models, the Log-linear model has been widely used because of its simplicity and ability to fit data well. For example, the Log-linear model has been used for modelling a worker's learning behaviour (Gruber, 1992), to measure progress in aerospace industry (Garvin, 2000), for life cycle estimation (Kortge et al., 1994), to investigate the effect of production breaks on 
production rates (Argote \& Epple, 1990; Jaber \& Bonney, 1996), and to investigate the effect of quality of a production process on performance (Jaber \& Guiffrida, 2004, 2008).

\subsection{Forgetting models}

Although there is enough evidences that forgetting occurs in organizations (Argote \& Epple, 1990; Benkard, 2000; Nembhard, 2000; Kleiner et al., 2011), there is no consensus among scientists on the factors that cause forgetting; however, production breaks are thought to be the primary cause of forgetting (Anderlohr, 1969; Cochran, 1973). Attempts to mathematically model the effects of forgetting in the industrial engineering literature are as early as the works of Hoffman (1968) and Adler and Nanda (1974).

Tight competence of professional firms and organizations, based on their knowledge and experience, makes forgetting and knowledge depreciation, a primary concern to many firms as it negatively affects the competitive advantage they have in a market (Karaoz \& Albeni, 2005). Measuring and evaluating this depreciation in knowledge, provide managers with strategies to sustain the levels of desired knowledge in their firms.

Modelling the forgetting behaviour falls in one of the three groups of mathematical, experimental, and empirical models. The works of Carlson and Rowe (1976), Globerson and Levin (1987), Elmaghraby (1990), and Jaber and Bonney (1996) fall in the first group with some of them were tested against experimental or empirical data. The works of Bailey (1989), Globerson et al. (1989), and Hewitt et al. (1992) are from the second group, where forgetting was modelled using data collected from laboratory experiments performed by students as surrogates of workers in manufacturing environments. Nembhard (2000) and Nembhard and Uzumeri 
(2000a) cautioned against using these models as they use single rather than multiple breaks when modelling forgetting. Badiru (1995) and Nembhard and Uzumeri (2000a) form the third and final group who modelled forgetting using empirical data collected from industrial settings.

The most common forms of forgetting curves are: Linear, Variable Regression Variable Forgetting (VRVF) or Power model (Carlson and Rowe, 1976), Hyperbolic (Mazur \& Hastie, 1978), Exponential (Globerson \& Levin, 1987; Mensik \& Raaijmakers, 1988), S-shape (Globerson \& Levin, 1987), Logarithmic (Woodworth, 1938), Exp-power (Wickelgren, 1972), Variable Regression Invariable Forgetting (VRIF) model (Elmaghraby, 1990), Learn Forget Curve Model (LFCM) (Jaber \& Bonney, 1996), Recency model (Nembhard \& Uzumeri, 2000a), Power Integration Diffusion (PID) model (Sikström \& Jaber, 2002), and Depletion Power Integration Latency (DPIL) model (Sikström \& Jaber, 2012).

\subsection{Fatigue models}

Grandjean (1979) has defined fatigue as "a loss of efficiency and a disinclination for any kind of effort". Fatigue was also defined as the difficulty in the initiation or sustainability of voluntary activities in clinical use (Chaudhuri \& Behan, 2004). Fatigue can be classified into two categories: Psychological (mental) and Physiological (muscular) (Nozaki et al., 2009).

Muscular fatigue is painful. It arises from overstressing muscles and reducing their tolerance to stress (Grandjean, 1979), which may indirectly lead to tissue damage or injury (Krajcarski \& Wells, 2008). Mental fatigue has been described by Grandjean (1979) as "a diffuse sensation which is accompanied by feelings of indolence and disinclination for any kind of activity". 
Fatigue, in a general sense, results from the mental and physical activities performed by individuals. It is alleviated during rest breaks including sleep (Grandjean, 1979; Sikström, 2011). Krajcarski and Wells (2008) showed that in systems with short production cycle times, workers recover from fatigue faster than systems with long ones, where less or no fatigue build up occurs across work shifts.

Rest breaks help alleviate body fatigue and recover a worker to his/her normal strength and capacity. In a flexible workforce such as DRC systems, workers alternate between different tasks that require various physical and mental workloads. When a worker performs a task, body fatigue is accumulated at a rate up to a threshold value (maximum endurance time). This fatigue is either alleviated by a rest break or by the worker moving to perform another task that has less/different physical demand allowing for some recovery. However, some of the learning acquired previously is lost when the worker is away from a task for some time (Kher et al., 1999; Kher, 2000).

Fatigue can be measured either by "objective" or "subjective" methods. For instance, the situation of mental fatigue is measured based on the self-report techniques and they are assumed to be "subjective" (Perez, 2011). Physical fatigue on the other hand, has been measured by many different "objective techniques" such as heart rate, electromyography, maximum endurance time, levels of oxygenation, and blood perfusion.

\subsubsection{Mental Fatigue models}

Nervous (mental) fatigue has been described as a function of several parameters such as temperature and moisture, noise, light, management weakness, mental involvement, 
forgetfulness, stress, job complexity, retraining, lack of team work, time constraints and vibration (Shahraki \& Bin Abu Bakar, 2011). There is no mathematical or quantitative model to measure mental fatigue; however, some scientists have tried to measure mental fatigue in experimental studies such as Murata et al. (2005), Vitório et al. (2012), and Gangopadhyay and Das (2012). Forgetting could be also considered as a result of mental fatigue.

\subsubsection{Physical Fatigue models}

Most existing physical fatigue models use either empirical or theoretical methods to approximate a worker's fatigue buildup over time. Empirical models usually use regression tools to derive the relationship between muscle fatigue and a task's quantifiable inquiries such as load and time (Xia \& Frey Law, 2008). Theoretical fatigue models, on the other hand, are usually applicable to specific groups of muscles and movements. These models can be divided into static and dynamic models. Static models usually use Maximum Endurance Time (MET) and Maximum Voluntary Contraction (MVC) as the key parameters to measure fatigue. El ahrache et al. (2006) compared twenty four MET models and concluded that there are significant discrepancies between $M E T$ models of back/hip, upper limb, or general muscles and $M V C$ limits that have been considered and reported by researchers. Dynamic models are used to determine the fatigue in dynamic work environments. For instance, Liu et al.(2002), Xia and Law (2008), and Law et al. (2012) have developed models for muscles' activation, and fatigue and recovery in dynamic loading conditions. Ma et al. (2009) developed a simple dynamic fatigue model considering the exerting load, workload history, and individual differences. This model is based on the hypothesis that maximum exertable force of the body declines with time, which is not being considered by static models. They validated their model mathematically with twenty four existing $M E T$ from static 
models (by considering the external load of the muscle constant) and three dynamic models (Freun's model for forearm (2001), Wexler's model for $\mathrm{Ca}^{2+}$ cross bridge mechanism (1997), and Liu et al. (2002). But this model has not been verified experimentally. The number of parameters to be determined, model complexity, and also restricted applications makes the existing muscle fatigue models either incomplete or unable to predict the general sense of fatigue in the body. In summary, fatigue measuring models are very different both in context and results and there is little agreement on the best approach.

\subsection{Recovery models}

Rest breaks are given to workers to have them recover from fatigue build-up. Determining the optimal length of the break time is one issue that has not been addressed correctly in the literature. As Mital et al. (1991) pointed out, the schedule and the length of break times are usually decided on through a management-union deliberations/agreements and are not based on any scientific or engineering rationale.

Many of the fatigue-recovery models have parameters such as maximum endurance time (MET) and maximum voluntary contraction $(M V C)$ levels, with percentages of them relative to the applied force or exposure time to be determined (Rohmert, 1973a, 1973b; Mital et al., 1991; Rose et al., 1992). Also, many of the models describe the rest allocations based on the fatigue of a specific part of the body (Iwanaga et al., 2000), which limits their applicability in more general cases such as entire body fatigue. The literature shows that there is no common consensus among researchers on how to schedule rest breaks and calculate their lengths (El ahrache et al., 2006). 


\subsection{DRC systems with learning and forgetting}

Learning, in an assembly job, can occur in the following steps: component identification, understanding the sequence of the assembly activities, and assembling of parts (Zamiska et al., 2007). When transferring between (different) jobs, a worker has the opportunity to practice his/her skills to maintain or improve his/her learning. However, in a DRC system, flexible workforce may suffer from forgetting as a result of alternating among different jobs. Previous studies indicate that productivity loss is a side effect of flexible workforce in DRC systems because of forgetting (Zamiska et al., 2007; Jaber \& Neumann, 2010). As the flexibility of a system increases, forgetting increases, which incur additional costs to the system since the worker, must re-learn the task that he/she revisits. Previous studies suggested that learning and forgetting must be modelled simultaneously in order to take advantage of the flexibility policies towards the organization's benefit (Kher et al., 1999). Several studies investigated the effect of flexibility and worker transfer policies in DRC systems to maximize the effect of learning and minimize that of forgetting. These studies consider periodic retraining, controlling the length of interruptions in the learning process, and utilizing the optimal level of flexibility, i.e. the number of tasks that a worker should be trained to perform (Kher et al., 1999; McCreery \& Krajewski, 1999; Zamiska et al., 2007). McCreery and Krajewski (1999) developed a model for an assembly line with learning and forgetting effects to investigate the use of workforce flexibility as a mean to improve the performance of the line. They provided cross-training policies to assign workers to tasks, and investigated the effect of product variety and task complexity. Using simulation and experimental design, they found that as task complexity increases, deployment of workers should be restricted and only low level of cross-training is needed. As the product variety increases, cross-training should be increased and deployment should be flexible. When both task 
complexity and product variety increase, cross-training should increase and deployment should be restricted. If task complexity and product variety are low, the authors found that a moderate amount of cross-training and flexible deployment is best.

Kher et al. (1999) and Kher (2000) studied worker training issues associated with learning and forgetting in DRC systems. They have used the learn-forget-learn model of Carlson and Row (1976) and found that in the presence of high forgetting rates, applying flexibility policies may not be feasible and if the forgetting rate is high (less than $85 \%$ ), then flexibility reduces worker efficiency. These studies suggest that in the presence of learning and forgetting, the benefit of worker flexibility is situational. If the flexibility cost is low, incremental flexibility improves the shop performance; however, if the learning losses are high, flexibility may worsen inventory and customer service performance. Also, they concluded that in the presence of low forgetting rates (90-95\%), upfront training does not impact the performance. Jaber et al. (2003) investigated the flexibility with the task similarity factor in the presence of learning and forgetting. They found that reducing the frequency of worker transfer to other tasks, reduces forgetting losses. They also observed that as task similarity increases, the importance of training and deployment decreases.

Yue (2005) studied workers flexibility in parallel DRC systems with learning and forgetting effects. They found that in the case of fast learning/forgetting, flexibility may not improve the performance of the system since more flexibility requires more learning and incur more forgetting. However, to manage the workload, a certain amount of flexibility is desired (Kim \& Nembhard, 2010). Jaber and Kher (2005) investigated workforce cross-training in DRC systems assuming that the production process may go out-of-control and produces defects that need rework. They have considered different patterns for learning in production and rework and 
examined the cross-training policies that produce less defective items. Their results indicated that for highly motor tasks, no upfront training is recommended and batch sizes decrease with less defects, which allows for more flexibility. Also they concluded that in $50 \%$ of cases, no upfront training is recommended.

In another study, Zamiska et al. (2007) applied a Dual-Phase Learning Forgetting Model (DPLFM) to consider the motor and cognitive contents of learning of a task. They have used the criteria defined by Dar-El et al. (1995) to include four different task types: purely motor, more motor than cognitive, more cognitive than motor, and purely cognitive. The authors compared their result with the previous study by Jaber et al. (2003), who utilized Learn Forget Curve Model (LFCM) to capture the learning and forgetting effects. Zamiska et al. (2007) indicated that the task's learning rate and proportion of cognitive and motor contents affect the performance of training and deployment policies in a DRC system. For instance, upfront training reduces the forgetting losses more effectively in the LFCM than in the DPLFM. Also, reducing the frequency of worker transfer, by reducing the forgetting effect, is more effective in the LFCM than the DPLFM. This study corroborates the results of Jaber et al. (2003), that it is not necessary to increase both the transfers and upfront training in the presence of low forgetting rates, since only one of them, suffices to counter the forgetting losses. For high forgetting rates, both transferring the workers and providing upfront training is necessary to confront the forgetting effects. In general, these two studies, indicate that the lower the learning rate or the $R$-value of the task ( $R$-value is defined as the ratio of the time for the first repetition under purely cognitive condition to the time for the first repetition under the purely motor condition), the greater is the need for a combination of reduced transfer policies or increased upfront training to confront the forgetting losses (Zamiska et al., 2007). 
Kim and Nembhard (2010) investigated the minimum staffing levels in parallel DRC systems in the presence of heterogeneous and individual learning and forgetting with a fixed production horizon. They studied worker selection policy, task heterogeneity (the variance among tasks with respect to task complexity), cross-training, time- schedule granularity (i.e. longer rotations mean fewer rotation over a given time horizon where finer granularity means shorter periods), and the production requirement. They observed that: first, a best workforce subset (the collection of best performing workers) requires fewer workers than an average subset (a collection of average performing workers). Secondly, restricting the extent of cross-training requires more workers. Thirdly, the time granularity does not influence the minimum staffing level.

Guimarães et al. (2012) studied the effect of rotation between tasks of different complexities on a workers' learning rate and performance. They have adopted two scenarios: the first scenario is when a worker transfers from an easy task to a difficult one. The second scenario describes the transfer of workers from a difficult task to an easy one. The level of task difficulty is determined by the task complexity according to an expert's assessment. They have measured the performance of workers in two scenarios in a pilot line in a Brazilian shoe factory using a 3parameter hyperbolic LC model of Mazur and Hastie (1978). Their results showed that there is no major difference in the performance of workers in the two groups and they concluded that task complexity does not change the workers' learning rate and performance. Following this result, they applied Macroergonomic Work Analysis (MA) analysis to a pilot line consisting of 100 workers. The MA method consists of applying training procedures and ergonomic improvements. To apply training procedures, workers with a good background were chosen to teach the task and its shortcuts to less skilled colleagues. Consequently, most workers in the pilot line, were able to perform more than 150 tasks in 3.5 years. Once workers were assumed to be 
multifunctional, they used task rotation procedures when every worker was able to change the workstation after 1.5 or 2 hours. In the multifunctional system, workers became indispensable and they were not worry about the quality problems of the assembly line, since it was the team responsibility not the person. The pilot line along with the introduction of some ergonomic improvements such as using personal protective equipment and water based glues, demonstrated $3 \%$ higher production, and reduction of $85 \%$ in rework. The rate of absenteeism reduced by $45 \%$, and Work Related Musculoskeletal Disorders (WMSD) and turnovers no longer were observed.

Azizi and Liang (2013) developed a model to assign working and training schedules and rotating workers between tasks while minimizing the total cost of training, flexibility, and productivity loss and solve the model by a two-phase heuristic. They have considered the workers' level of skill by their learning and forgetting, and investigated the effect of rotation intervals on the skill variation which affects the training costs subsequently. Their model also includes the minimum residency time (the time that worker must spend on a task before being eligible to transfer to another task) and heterogeneous flexibility, i.e. different levels of task proficiency. Their findings indicated that the length of the rotation interval has a significant impact on the total cost and the shorter the interval, the higher is the total cost. Also, they observed that when the multifunctionality is low and uniformly distributed among workers, better results are obtained. The results of Azizi and Liang (2013) indicated that longer residency time and rotation interval, enhance a worker's skills by learning. Also, reducing the level of multi-functionality, by providing equal opportunities for workers to learn and enhance their skill level, incur to lower costs. 
Previous studies, including the above mentioned, have largely contributed to understanding the behaviour of DRC systems in the presence of learning and forgetting. However, the literature suffers from a lack of attention towards human effects on DRC systems. Although, learning and forgetting, are important aspects of human involvement, other aspects such as fatigue and recovery, human error, and its influence on the quality of final products have not been considered by the above mentioned researches. In the following sections, a literature review considering human factors in production planning and DRC systems is presented.

\subsection{DRC systems with human factors}

The ratio of the number of workers to the number of machines expressed as a percentage between $0 \%$ and $100 \%$ is defined as the "staffing level" (Lobo et al., 2014). Since the staffing level in DRC systems is usually less than 100\% (Jaber \& Neumann, 2010), workers are transferred between tasks to fulfil the orders and share the workload. A flexible workforce reduces lead times and inventories, and also reduces fatigue, boredom, repetitive stress, and injuries (Hopp \& Oyen, 2004; Jorgensen et al., 2005).

Carnahan et al. (2000) developed an integer programming model for job scheduling to reduce the potential of back injury in the workers. The schedules consisted of lifting tasks whose potential for causing injury was assessed by a job severity index provided by Liles (1986). Using a genetic algorithm, they created job rotation schedules to maintain the productivity while controlling the exposure to musculoskeletal strain. Tharmmaphornphilas et al. (2003) developed a model to reduce the likelihood of worker hearing loss by rotating the workers through different jobs during the day. They performed a computer simulation based on the data collected from a real

setting and used noise regulations of the National Safety Institute of Occupational Safety and 
Health (NIOSH) concerning safe duration of noise exposure. They were able to reduce the maximum daily dose of time-weighted average sound level that any worker was exposed to by $58.8 \%$ with improved schedules.

In another study, Aryanezhad et al. (2009) investigated safe skill-based job rotation scheduling by integer programming to simultaneously minimize the maximum occupational noise exposure injuries and the potential of worker's low back pain. They assessed the noise exposure level of each job by daily noise dosage and the potential for low back injury by job severity index provided by NIOSH regulations. They found that considering only one objective (noise dosage or back injury), may sacrifice the other one. The model was able to provide a trade-off between both objectives; however, in some cases, finding the feasible solution was not possible and allowed noise exposure or job severity level exceeded. They concluded that in real cases, it may be impossible to take predefined thresholds for noise exposure or job severity into consideration because of the nature of the job and types of machines. Lodree Jr et al. (2009) conducted a job scheduling accounting for physical and/or cognitive human characteristics. They identified human characteristics related to task sequencing that could establish a framework for that purpose for any working environment with productivity and safety objectives that is characterized by demanding and various tasks. They argued that learning-forgetting, performance measurement related to accuracy (quality), fatigue and cumulative workload, as well as individual differences and limits, play an important role in task sequencing. Therefore many of the traditional scheduling methods are not applicable when tasks performed by human resources. Authors asserted that the integration of elements of classical scheduling models with human performance modeling leads to the better characterization of the task outcome. 
While the work of Lodree Jr et al. (2009) provides an important milestone in job scheduling involving human, the effect of the excessive flexibility and visiting several work stations with different physical demands in increasing the exposure of the workers to fatigue, injuries, or error committing is not clear. There are very few studies that investigated the risk of fatigue or injury as a result of job rotation or considered them in solving job rotation problems. The next section provides a literature review of DRC studies that considered human fatigue and recovery.

\subsection{DRC systems with fatigue and recovery}

High loads and recurrent or prolonged loading generate fatigue that is a source of error, accidents, and quality issues at any involvement level such as operation, maintenance, engineering, and management (Dinges, 1995; Sherman, 2003; Dionisio, 2010; Bevilacqua et al., 2012). Fatigue is usually alleviated by transferring a worker to a physically less demanding task or by giving the worker a rest break (Jaber \& Neumann, 2010; Horton et al., 2012). Although fatigue has been identified as a potential research area in many recent studies, fatigue and recovery have not received much attention in the Operations Research literature and the studies that captured the effects of worker fatigue on the production output and the quality of work are scarce. Fatigue is more concerned in DRC systems where workers transfer between tasks with different workload. In these systems, the production planning schedule should provide the workers with the required recovery, which is accomplished by either rest breaks or transferring workers from jobs with higher to the ones with lower workloads. Therefore, the traditional production planning might be revised in this context to avoid overloading workers and fulfilling the demand at the same time. 
There are very few studies that have utilized workers fatigue and recovery as a constraint for obtaining transferring schedules. The study by Jaber and Neumann (2010) is believed to be the first to model worker fatigue and recovery in a DRC job shop system to address the flexibility issues associated with the workload. Considering a linear model for physical fatigue, i.e. fatigue increases linearly with time, Jaber and Neumann (2010) developed a Mixed-Integer Linear Programming (MILP) model in which the objective function is a combination of productivity and physical loading where their importance or weights is determined by a manager, union, or analyst. In this model, as productivity increases, with the number of workers constant, the work load on the workers and accordingly their fatigue increases. Minimizing the above objective function with respect to the time and fatigue constraints will yield the optimum schedule. To simplify the MILP model, the authors considered four practical cases with combinations of two tasks with breaks. The results suggested that if productivity is preferred over fatigue, full recovery after the second task is recommended. On the other hand, if minimum fatigue is preferred over productivity, full recovery after each task is recommended. Their results also suggested that having a shorter residence time (minimum time to spend on each task before the worker transfers to another task) improves the overall performance of the system by shortening the cycles that generate fatigue which can be recovered in shorter breaks. The recovery rate and the load level also showed to affect the system performance, i.e. faster recovery and an unequal load level for the two tasks (fraction of maximum load) improve the system performance.

Following Jaber and Neumann (2010), Othman et al. (2012) developed a workforce scheduling model including human aspects such as skill level, training, personalities, and worker fatigue and recovery. Considering four different objective functions to minimize the cost, firing the high performance workers, fatigue, and idle time, they have created a multi-objective integer 
programming model for staffing decisions in a heterogeneous workforce in which workers have different personality and skill levels. They observed that fatigue is not significantly important for day scheduling purposes from an economic perspective but it may be utilized to determine the required break time for the worker. Azizi et al. (2010) modelled job rotation in cycles of motivation and boredom of the worker while considered the effects of learning and forgetting on skill variation. They attempted to balance the positive aspects of the job rotation with the associated costs of skill deterioration as a result of forgetting. They minimized the total delay during a production horizon caused by worker's boredom and/or skill deterioration. The results of their study show that the total delay significantly decreases when a partial or complete rotation plan applies. However, if the skill improvement is prioritized over the worker motivation (i.e. the primary objective is to improve the skills), then the job rotation plan may even increase the total tardiness. This result is interesting since by transferring between different jobs, the worker has less time for skill improvement and may make more errors that generate items that require rework. This suggests that the effect of transfer policies on product quality should be considered when modelling DRC systems (Pinker \& Shumsky, 2000).

\subsection{DRC systems and quality of the products}

Most of the DRC system studies consider the output (products) to have a perfect quality. However, this is not the case in real industrial environments where defective items are generated by machine malfunction or human error. The author found two studies that considered product quality with cross-training and rotation policies in DRC systems. The first study is the one by Jaber and Kher (2005) who investigated quality issues resulting from machine malfunction. They investigated cross-training policies in a DRC system with an imperfect production process where 
the generated defective items are reworked. They adopted the Porteus (1986) model to simulate the situation where production process deteriorates from an 'in-control' state to an 'out-ofcontrol' state with defective items produced as a result. The result of this study was presented in sub-section 2.5. The second study is that of Michalos et al. (2013) who considered human error in producing quality deficits by investigating the effect of job rotation on the quality of the final product in a human-based assembly environment. They have modelled human error with task repetitiveness, competency level, and physical fatigue. The result of their study demonstrated that the quality of the final product is affected by human fatigue, which causes errors. The findings also indicated that applying job rotation significantly enhances product quality and reduces errors.

\subsection{Research Gaps}

The above literature review shows that there is no study that investigates a DRC system in the presence of learning, forgetting, fatigue, recovery, quality, and human error. Table 2-1 identifies the research gaps in the literature by identifying human aspects each study considered and whether they were considered in a DRC setting. The table clearly depicts the research gaps (empty cells) and the contribution of this thesis to the literature. 
Table 2.1. The contribution of the proposed research to the industrial engineering literature.

\begin{tabular}{|c|c|c|c|c|c|c|c|c|c|}
\hline Author \& & 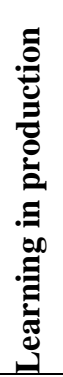 & 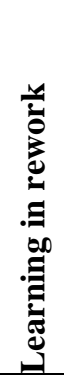 & 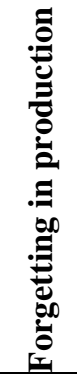 & 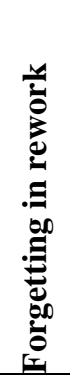 & 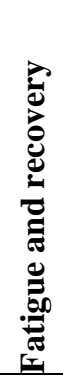 & 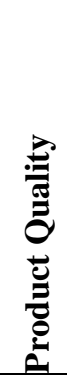 & 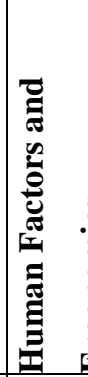 & 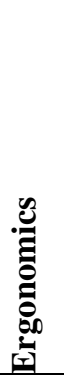 & 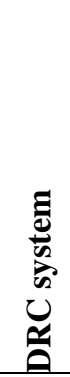 \\
\hline Porteus (1986) & & & & & & $\checkmark$ & & & \\
\hline Jaber and Bonney (1996) & $\checkmark$ & & $\checkmark$ & & & & & & \\
\hline Konz (1998) & & & & & $\checkmark$ & & & & \\
\hline Kher et al. (1999) & $\checkmark$ & & $\checkmark$ & & & & & & $\checkmark$ \\
\hline Jaber et al. (2003) & $\checkmark$ & & $\checkmark$ & & & & & & $\checkmark$ \\
\hline Jaber and Guiffrida (2004) & $\checkmark$ & $\checkmark$ & & & & $\checkmark$ & & & \\
\hline Khouja (2005) & & & & & & $\checkmark$ & & & \\
\hline Jaber and Kher (2005) & $\checkmark$ & $\bar{\checkmark}$ & & & & $\checkmark$ & & & $\checkmark$ \\
\hline Jaber and Guiffrida (2008) & $\checkmark$ & $\checkmark$ & & & & $\checkmark$ & & & \\
\hline ElMaraghy et al. (2008) & & & & & & & $\checkmark$ & 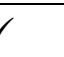 & \\
\hline Ma et al. $(2009)^{1}$ & & & & & $\checkmark$ & & & & \\
\hline Jaber and Neumann (2010) & & & & & $\checkmark$ & & & & $\checkmark$ \\
\hline Myszewski (2010) & & & & & & & $\checkmark$ & 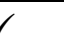 & \\
\hline Myszewski (2012) & & & & & & & $\checkmark$ & r & \\
\hline Guimarães et al. (2012) & $\checkmark$ & & & & & & $\checkmark$ & I & $\checkmark$ \\
\hline Glock and Jaber (2013b) & $\checkmark$ & $\checkmark$ & $\checkmark$ & $\checkmark$ & & & & & \\
\hline Michalos et al. $(2013)^{2}$ & & & & & $\checkmark$ & $\checkmark$ & & & \\
\hline This thesis & $\checkmark$ & $\checkmark$ & $\checkmark$ & $\checkmark$ & $\checkmark$ & $\checkmark$ & $\checkmark$ & 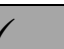 & $\checkmark$ \\
\hline
\end{tabular}

${ }^{1}$ Ma et al. (2009) only considered fatigue and not recovery.

${ }^{2}$ Michalos et al. (2013) only considered fatigue and not recovery. 


\section{Framework and Methodology}

This section provides the framework and methodology for this research. The research is performed in four Phases, each with distinct objectives. Each Phase is intended to respond to some research questions while it contributes to the entire body of the thesis by completing the previous Phases.

\subsection{Phase 1: Incorporating fatigue-recovery into the learning-forgetting model}

The available learning and forgetting models do not consider the physical loading that performing a task requires. In some situations, physical loading results in workers' fatigue on the job that is followed by rest breaks to alleviate it. The aim of this Phase of the thesis is to present the "Learning Forgetting Fatigue Recovery Model" (LFFRM) that addresses possible issues relating to workers' capabilities and restrictions in manufacturing environments. Numerical examples are solved to address some research questions regarding the model optimization and its constraints. The main results show that incorporating learning into a production process decreases fatigue and improves the performance of the system. Worker's fatigue, on the other hand, increases the production time and decreases production outputs. A recovery break must be of enough length to alleviate some of the accumulated fatigue; however, longer recovery times extend the lead-time and deteriorate the production performance due to forgetting.

This Phase of the thesis investigates how system throughput is influenced by human behaviour. It also provides solutions to improve the system performance as well as workers' wellbeing. The results of this Phase have been published in the following paper: 
Jaber, M. Y., Givi, Z. S., Neumann, W. P. (2013), Incorporating human fatigue and recovery into the learning-forgetting process. Applied Mathematical Modelling, 37 (12-13), 7287-7299.

\subsection{Phase 2: Incorporating forgetting into the quality learning curve}

Industries spend significant amounts of money to counter the effects of forgetting by training and retraining workers. Forgetting increases the process cost and hinders improvements in the performance. This Phase of the thesis provides insights into the effect of forgetting on the production process in which some produced items are not considered to have perfect quality and therefore must be reworked. This also has important implications in reverse logistics where most processes are labour intensive (Jaber \& Bonney, 2011).

The Wright's learning curve (WLC), which is the most utilized learning curve, assumes that every unit of production has an acceptable level of quality, which is not the case in many production environments. Many studies reported that a production process may go out-ofcontrol, thus generating defective items requiring rework. Jaber and Guiffrida (2004) have modified the WLC by accounting for rework time. In a later study, Jaber and Guiffrida (2008) allowed for production interruption to restore the quality of the production process to reduce the number of defective per lot. Although these works were the first analytical models that linked learning to quality, their results cannot be generalized as they considered a single (first) production cycle. This assumption ignores the transfer of learning that occurs between cycles in intermittent production environments. Phase 2 of the thesis addresses this limitation and considers the knowledge transferred to deteriorate because of forgetting. The results indicate that the performance function of the process has a convex form under certain conditions. The 
performance of the system improves with faster learning in production and rework, frequent process restorations, and transfer of learning between cycles.

This Phase also considers quality deterioration in the production line where the process generates defective items and is influenced by worker's learning and forgetting. The system performance measure used is the time required to produce a working unit. If it is a defective item, then it is reworked. An optimization method is applied to determine the optimal lot size.

Phase 2 of this research sets strategies for quality improvements, waste reduction, and improving the standard time (breaking the learning plateau). The results are useful in scheduling workers in the DRC systems where there is a trade-off between quality and productivity. The results of this Phase were presented at the $10^{\text {th }}$ Computational Management Science (CMS) conference in Montréal, QC, in May 2013. Also, an extended version of the conference paper is found in:

Jaber, M.Y., Givi, Z.S. (2014). Imperfect production process with learning and forgetting effects, Computational Management Science (DOI: 10.1007/s10287-0140205-y).

\subsection{Phase 3: Investigating human error with learning-forgetting and fatigue-recovery}

Recent studies have shown that automation does not necessarily decrease human involvement in manufacturing, nor does it reduce the worker's physical workload. As a matter of fact, there are still numerous assembly jobs that require human involvement. Therefore, human performance continues to have a significant impact on the quality and cost of products.

While modelling human error has been investigated in the engineering literature for many years, the existing models are not capable of anticipating how and when an error occurs. This Phase of 
the thesis studies the confluence of learning-forgetting and fatigue-recovery factors that affect human reliability when performing a manual task. Human error is defined as a mistake in performing a task that results in producing a quality deficit, which is corrected at a cost and it is considered to be affected by the worker's personal capabilities and the work characteristics. The learning curve represents the worker's capability in performing a repetitive task, or competence. Also, the effect of the work specifications on the worker's outcome is presented by worker's fatigue which is directly affected by the work environment. A mathematical model is developed that estimates the human error rate while performing an assembly job and accounts for the dynamic behavior of human reliability in a production process. This model measures and optimizes the production throughput with regard to human reliability.

The preliminary results of this Phase were presented at an IIE Annual Conference as:

Givi, Z.S., Jaber, M.Y., (2014) Human error due to learning and fatigue. Paper presented at the Proceedings of IIE Annual Conference and Expo 2014, May 31June 03, Montréal, Canada.

An extended and full version of this paper was submitted for review and is cited here as:

Givi, Z.S., Jaber, M.Y., Neumann, W.P. (Submitted) Modelling worker reliability with learning and fatigue. Journal of Applied Mathematical Modeling.

\subsection{Phase 4: Investigating flexibility and transfer policies in a DRC system with some human factors}

This Phase of the thesis develops a DRC system model and integrates it into the Learning Forgetting Fatigue Recovery Model (LFFRM), the Quality Learning Forgetting Curve (QLFC) 
models, and human/process error to examine the system performance. Conflicting system objectives arise when the effects of learning-forgetting and fatigue-recovery are combined. Some of the parameters such as learning, recovery, and flexibility, enhance the system performance, while others weaken it such as fatigue, excessive flexibility, forgetting, and defects. Phase 4 completes this thesis by presenting an inclusive model for a DRC system which is capable of relating the system characteristics (flexibility and transfer policies) to human behavior (learningforgetting, fatigue-recovery, and error making). The developed model could be used for quality improvement and for deriving strategies for production planning and job scheduling. The results of this Phase were submitted to the following peer-reviewed journal for review:

Givi, Z.S., Jaber, M.Y., Neumann, W.P. (Submitted) Production planning in DRC systems with human intervention, Journal of Computers and Industrial Engineering. 


\section{Phase 1: Incorporating fatigue-recovery into the learning-forgetting model}

In Phase 1, the Learn Forget Curve Model (LFCM) is modified to account for the effects of fatigue and recovery. The "Learning Forgetting Fatigue Recovery Model" (LFFRM) is developed, which investigates the interaction of workers' physical capabilities with learning and forgetting. This Phase of the thesis provides solutions to improve the system performance as well as workers' wellbeing. The results of this Phase will be used to capture the influence of human behaviour in production planning in Phase 4.

\subsection{Introduction}

The "Learning Curve" (LC) has been an interesting subject to many researchers and practitioners including industrial engineers and psychologists since the work of Wright (1936), and Hovland (1951). Since then, several learning curve models were developed that have different forms (e.g.,

power exponential, S-curve). The Wright's learning curve has been the prominent model because of its simplicity and ability to fit well a wide range of learning data (Yelle, 1979; Badiru, 1992; Jaber, 2006).

Globalization and competition have changed the market environment and imposed pressures on manufacturing firms to deliver new and quality products at competitive prices more frequently, requiring these firms to be responsive, efficient, and flexible. To cope with these changes, manufacturing firms started acquiring flexible workforce approaches to alleviate the detrimental effects of bottlenecks resulting from machine breakdowns, product type changes, or external demand changes. The flexible workforce can also reduce work-in-process inventory levels, leadtimes, and improve customer service performance (Park \& Bobrowski, 1989). However, flexibility comes at the cost of the workforce partially or fully forgetting the knowledge acquired 
for one task while performing different tasks (Jaber et al., 2003; Inman et al., 2004). Automotive manufacturers are an example where significant amounts of money have been spent on training (learning) and retraining (relearning following forgetting) workers (Park \& Bobrowski, 1989; Omar et al., 2011). This enticed many researchers to investigate how learning and forgetting interacts, and to develop models that capture these phenomena (Bailey, 1989; Jaber \& Bonney, 1997; Kleiner et al., 2011).

In environments that emphasize flexibility, forgetting, which may be thought of as the loss of access to knowledge or procedures, occurs either because a worker has been away from a given task for some time or because he/she confuses similar knowledge or skills (Bjork \& Bjork, 2011). In the industrial engineering literature, it has been documented that the length of a production break has a direct effect on the degree to which humans forget (Anderlohr, 1969; Cochran, 1973) and it may occur over relatively short periods of time (MacLeod \& Macrae, 2001; Anderson, 2003). Modelling the forgetting curve falls in one of the mathematical, experimental, and empirical groups as stated in Section 2.2 where the "Learn Forget Curve Model" (LFCM) was found to be a promising model (Jaber \& Bonney, 1997; Jaber et al., 2003; Jaber \& Sikström, 2004a, 2004b). The models of the three groups were most likely investigated in environments where a worker may be subjected to fatigue on the job. There is evidence in the literature that repetitive human daily activities deplete an individual's resources leading to fatigue (Winwood et al., 2005; Sonnentag \& Zijlstra, 2006). It is reasonable therefore to assume that workers in an industrial setting are subject to fatigue on the job.

Fatigue is multidimensional. Tiredness and lack of energy (Barker \& Nussbaum, 2011), physical exertion (Barker \& Nussbaum, 2011), physical discomfort (Yoshitake, 1978), lack of motivation 
(De Vries et al., 2003), and sleepiness (Smith et al., 2005; Theorell-Haglöw et al., 2006) have been distinguished as dimensions of fatigue (Åhsberg, 2000). Fatigue is a common result of work (Winwood et al., 2005) and leads to performance problems. It has detrimental effects on judgment, omission of results, indifference to essentials, decreased efficiency and productivity, and higher error rates and quality problems (Rohmert, 1987; Åhsberg, 1998; Chaffin et al., 2006; ElMaraghy et al., 2008). When fatigue becomes chronic or excessive, it reduces a person's quality of life and can contribute to work-related disorders (Åhsberg, 1998; Frank, 2000; Asfaw et al., 2011; Bevilacqua et al., 2012). Rest breaks help alleviate body fatigue and recover a worker to his/her normal strength and capacity.

In a flexible workforce schedule, workers alternate between different tasks that require various workloads. When a worker performs a task, muscle force capacity is reduced by time up to a threshold value (maximum endurance time) due to the muscle fatigue (Ma et al., 2009). This fatigue is either alleviated by a rest break or by the worker moving to perform another task that has less and different physical loading allowing for some recovery. However, some of the learning acquired previously is lost when the worker has been away from a task for a period of time. Although fatigue has been considered widely in the ergonomic literature, it has not received much attention in the Operation Research literature. There is no study available that demonstrates the mathematical relation between worker fatigue and production outputs such as production time and volume.

Conflicting system objectives arise when the effects of learning-forgetting and fatigue-recovery are combined. On one hand, the system productivity improves as workers move down on their learning curves by producing more per unit time; although their ability to work for long periods 
of time is constrained by muscular fatigue. On the other hand, a rest break or transferring a worker to a different task alleviates fatigue and recovers a worker's physical condition to a comfort state (Jaber \& Neumann, 2010); however, it may impede the worker's productivity because of forgetting. There is no available model in the learning-forgetting literature that accounts for human fatigue and recovery. The aim of this Phase is to present the "Learning Forgetting Fatigue Recovery Model" (LFFRM) that addresses possible issues relating to workers' capabilities and restrictions in manufacturing environments. The rest of this section is organised as follows. Section 4.2 presents the mathematics of the "Learn Forget Curve Model (LFCM)", followed by a brief introduction to human fatigue and recovery models in 4.3. The mathematics of the integrated LFFRM is developed in 4.4 followed by methodology in 4.5 . Numerical examples are provided in 4.6 with their results discussed to address several research questions. Section 4.7 addresses the limitations and feasible solutions of the developed models and the last section, section 4.8 , is for discussion and conclusions.

\subsection{Mathematics of the LFCM}

Wright's (1936) learning curve suggests that the time to perform a task decreases by a constant percentage each time the cumulative output doubles, and it is represented by:

$T_{x}=T_{1} x^{-b}$

where $T_{x}$ is the time to produce the $x^{\text {th }}$ unit, $T_{1}$ is the time to produce the first unit, $x$ is the cumulative production, and $b$ is the learning index $(0 \leq b<1)$, where $b=-\log (L R) / \log (2)$ and $L R$ is the learning rate measured in percentage $(0<L R<1$; e.g. $L R=0.8$ or $80 \%)$. 
Equation (4.1) is hypothesised by Wright (1936) theory that the labor-hour required to perform a unit of job, decreases by a constant percentage every time the repetition quantity is doubled. Typical learning rates according to Crawford (1944) are shown in Table 4.1.

Table 4.1. Typical learning rates adapted from Crawford (1944), higher rates represent slower learning.

\begin{tabular}{llll}
\hline Type of work & LR \% & Industry & LR \% \\
\hline ASSEMBLY & $84-85$ & AEROSPACE & 85 \\
PROTOTYPE ASSEMBLY & 65 & COMPLEX MACHINES & $75-85$ \\
CLERICAL OPS & $75-85$ & CONSTRUCTION & $70-90$ \\
INSPECTION & 86 & ELECTRONIX MFG & $90-95$ \\
MACHINING & 90 & MACHINE SHOP & $90-95$ \\
WELDING & $85-90$ & SHIPBUILDING & $80-85$ \\
\hline
\end{tabular}

Similar information to the one in Table 4.1 can be found in Dutton and Thomas (1984) and DarEl (2000). The forgetting phenomenon has been considered to be a mirror image (Globerson et al., 1989) of the learning curve and it is represented by:

$\hat{T}_{x}=\hat{T}_{1} x^{-f}$

where $\hat{T}_{x}$ is the time for the $x^{\text {th }}$ unit of lost experience on the forgetting curve, $\hat{T}_{1}$ is the intercept of the forgetting curve, $x$ is the amount of output that would have been accumulated if interruption did not occur and $f$ is the forgetting index. Figure 4.1 shows the learning-forgetting process. 


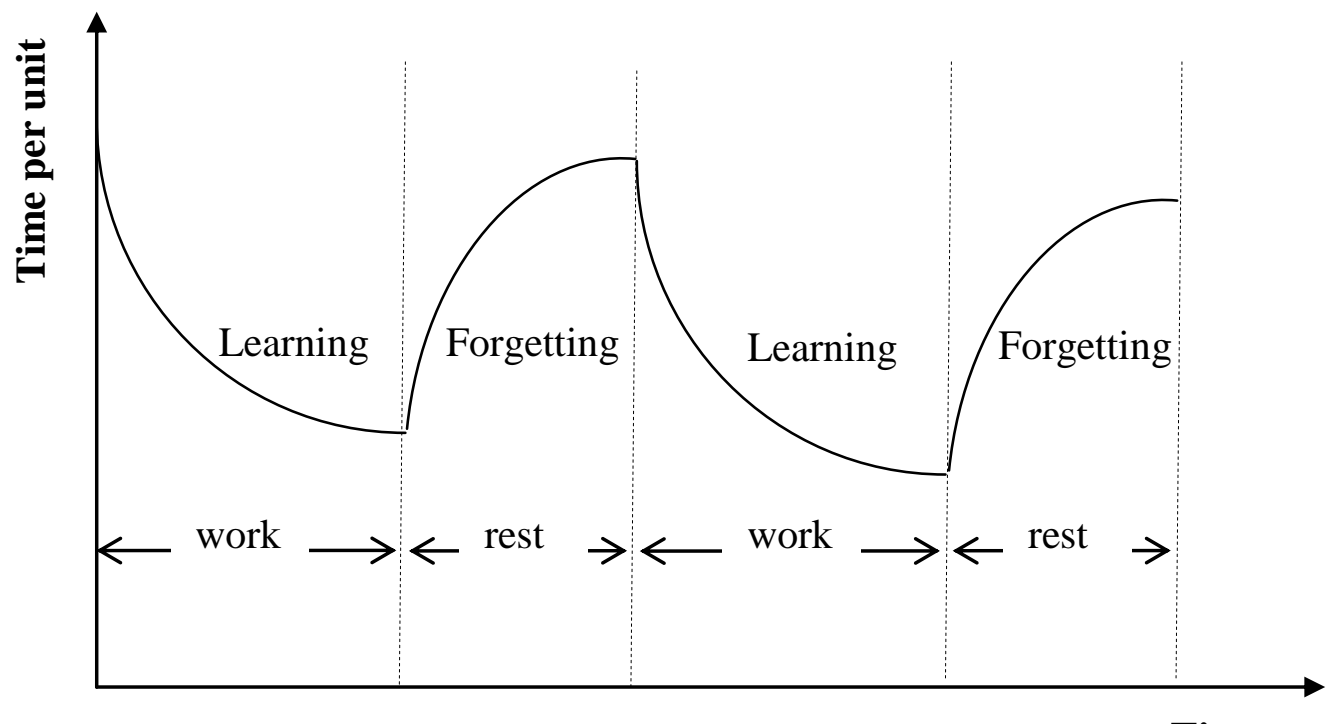

Time

Figure 4.1. An illustration of the behaviour of the learning-forgetting process over repeated work-rest cycles.

Using equations (4.1) and (4.2), Jaber and Bonney (1996) developed the "Learn Forget Curve Model" (LFCM) which is represented by:

$T_{x_{i}}=T_{1}\left(u_{i}+x_{i}\right)^{-b}$

where $T_{x_{i}}$ is the time to produce the $x^{\text {th }}$ unit in cycle $i, u_{i}$ is the experience measured in the number of units remembered at the beginning of cycle $i$, and $x_{i}$ is the number of repetitions (units produced) in cycle $i$. The term $u_{i}$ is determined from the work of Jaber and Bonney (1996) as: 
$u_{i+1}=\left(u_{i}+x_{i}\right)^{1+f_{i} / b}\left(u_{i}+x_{i}+S_{i}\right)^{-f_{i} / b}$

where $u_{1}=0, S_{i}$ is the number of units of a product that could have been produced in cycle $i$ if production interruption did not occur. The terms $S_{i}$ and $f_{i}$ are given respectively as:

$S_{i}=\left[\frac{1-b}{T_{1}} \varphi_{i}+\left(u_{i}+x_{i}\right)^{1-b}\right]^{1 /(1-b)}-\left(u_{i}+x_{i}\right)$

$f_{i}=\frac{b(1-b) \log \left(u_{i}+x_{i}\right)}{\log \left(1+B / t\left(u_{i}+x_{i}\right)\right)}$

where $\varphi_{i}$ is the length of the interruption period that has occurred after producing batch $i, B$ is the time to which total forgetting occurs $\left(\varphi_{i} \leq B\right)$, and $t\left(u_{i}+x_{i}\right)$ is the time to perform (produce) $u_{i}+x_{i}$ repetitions (units) continuously on the learning curve. Assuming that total forgetting can occur is not unrealistic given that it was observed in the studies of Anderlohr (1969), McKenna and Glendon (1985), and Globerson et al. (1998).

\subsection{Fatigue and recovery models}

Fatigue can take many forms such as mental fatigue, lack of alertness, specific muscular fatigue, or general body fatigue (Åhsberg, 1998). The effect of fatigue on performance has been documented across many industrial and service sectors, e.g., the loss of throughput (Wang \& $\mathrm{Hu}$, 2010), error increasing (Misawa et al., 1984; Kopardekar \& Mital, 1994), worker's 
dissatisfaction (Saijo et al., 2008), performance decrement (Mital et al., 1991; Goode, 2003; Leung et al., 2006; Barker \& Nussbaum, 2011), and injuries and accidents (Schuster \& Rhodes, 1985; Dinges, 1995; Kristal-Boneh et al., 1996; Muggleton et al., 1999; Burke \& Fiksenbaum, 2008). However, a mathematical model that relates fatigue and production outcomes has not been introduced. This thesis focuses on muscular fatigue which is defined as the inability of the body muscles to sustain a specific posture or force level required to perform a task (Ma et al., 2009). A model is developed to quantify the relation between worker's muscular fatigue and production outputs.

Maximum Endurance Time (MET) is the duration for which a specific body posture (or muscular effort) can be sustained (applied) by a worker before his/her capability limits are reached (Niebel et al., 1999; El ahrache et al., 2006). It is a function of the level of the force being applied, e.g., $\left(f_{\mathrm{MVC}}\right)$. This force is usually a fraction of the muscle's Maximum Voluntary Contraction $(M V C)$ when performing a specific task (El ahrache et al., 2006), e.g., $f_{\mathrm{MVC}}=\% M V C$. Readers may refer to El ahrache et al. (2006) or Ma et al. (2009) for a good review of a number of empirical models that predict $M E T$. The available models are either of exponential forms, e.g., $M E T=\beta_{0} \times e^{-\beta f_{\mathrm{NwC}}}$ (Rose et al., 1992), or power forms, e.g., $M E T=\alpha_{0} \times f_{\text {MVC }}^{-\alpha}$ (Sjøgaard, 1985), where $\alpha_{0}, \alpha, \beta_{0}$ and $\beta$ are model-specific parameters and MET is measured in minutes. These MET models are limited in the sense that they predict a fatigue end-point under a given $f_{\mathrm{MVC}}$ load but provide no indication to the shape of the fatigue accumulation function or depict the fatigue state over the course of the task execution.

Although some researchers asserted that fatigue accumulates exponentially with time (Lindstrom et al., 1977; Bechtold \& Sumners, 1988; Konz, 1998), none have empirically or experimentally 
showed that it does. This remains an open research question to be addressed in the Human physiology literature. On the contrary, more attention was paid by researchers in ergonomics to the form of the recovery function, which Konz also suggests is exponential (Konz, 1998), with maximum benefit in the earlier phases of the recovery period. Readers may refer to El ahrache and Imbeau (2009) for a review of recovery models. As suggested by Konz (1998), this thesis assumes that fatigue and recovery are of the following forms respectively:

$F(t)=1-e^{-\lambda t}$

$R\left(\tau_{i}\right)=F(t) e^{-\mu \tau_{i}}$

where $F(t)$ is the fatigue accumulation at time $t \leq M E T$, and $R\left(\tau_{i}\right)$ is the residual fatigue after a rest break of length $\tau_{i} \geq 0 ; R=0$ represents complete recovery (no residual fatigue) and $R=1$ represents no recovery (maximum fatigue). Since the learning rest break $\varphi_{i}$ in equation (4.5) in this Phase is equal to the recovery rest break $\tau_{i}$ in equation (4.8), only in this Phase of the thesis, both are referred to $\tau_{i}$. In (4.7) and (4.8), $\lambda$ and $\mu$ are fatigue and recovery parameters, respectively. These parameters control the rate of fatigue accumulation and recovery alleviation; i.e., a low value of $\lambda(\mu)$ means slow fatigue accumulation (recovery) whereas a high value means a fast one. Figure 4.2 illustrates the behaviour of a fatigue-recovery process. 


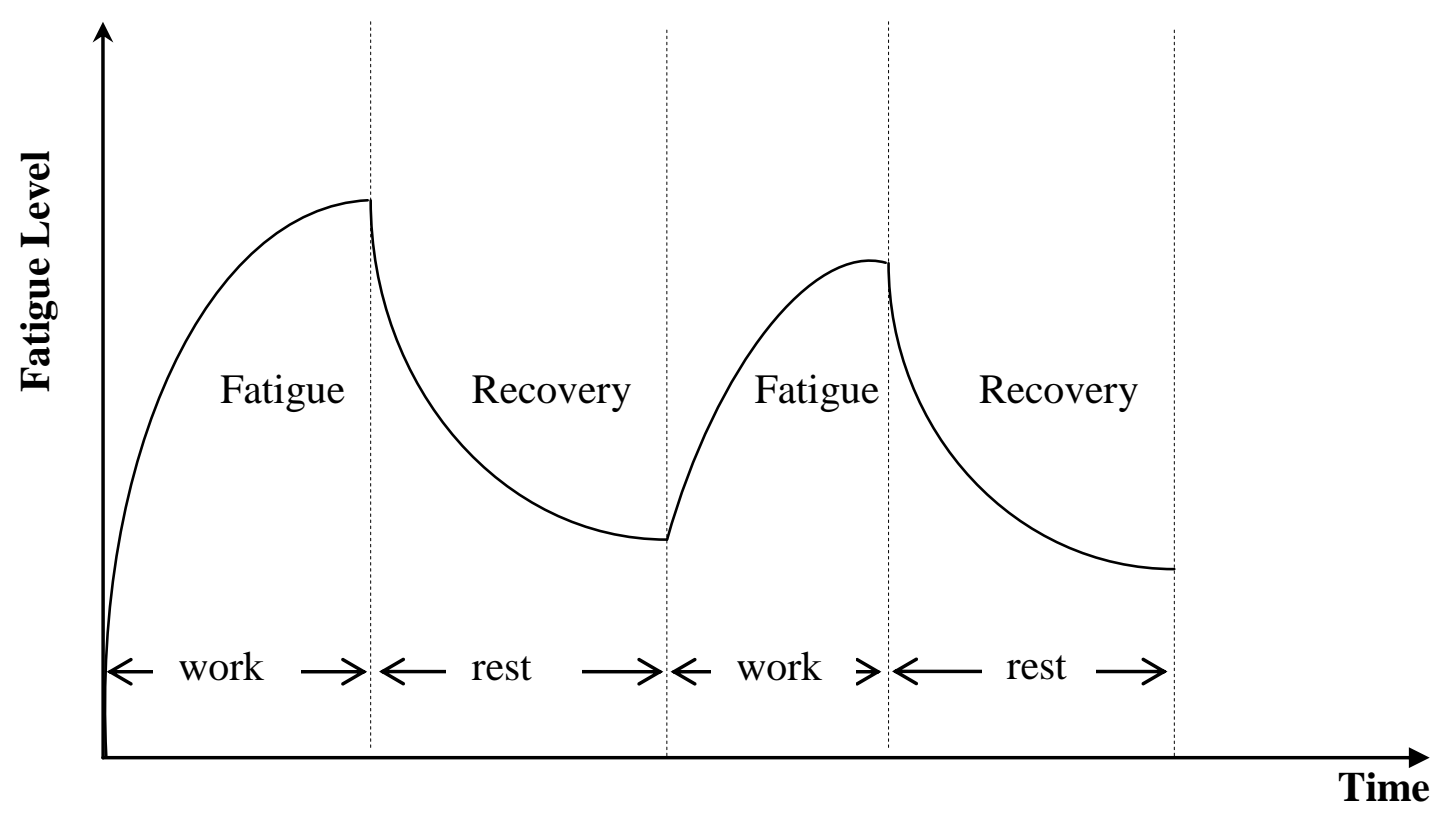

Figure 4.2. An illustration of the behaviour of the fatigue and recovery process over repeated work-rest cycles.

Note that equation (4.7) advocates that fatigue accumulates over $t$ from an initial value of zero, meaning that full recovery was attained in the previous rest break. In practice, rest breaks separating work cycles are usually short and do not permit full recovery to occur. The residual fatigue $R\left(\tau_{i}\right)$ is carried forward from cycle $i$ to cycle $i+1$ and equation (4.7) is re-written as:

$F_{i+1}(t)=R\left(\tau_{i}\right)+\left(1-R\left(\tau_{i}\right)\right)\left(1-e^{-\lambda\left(t_{x}-t_{i}\right)}\right)$

where $t_{x}$ is the production time of the cycle $i$ and $t_{i}$ is determined by projecting the value of $R\left(\tau_{i}\right)$ on the fatigue curve as: 
$t_{i}=-\ln \left(1-R\left(\tau_{i}\right)\right) / \lambda$

A dynamic fatigue model (Ma et al., 2009) is used to consider different maximum endurance times $(M E T)$ for different production batches as a result of fatigue accumulation. This assumption is based on the hypothesis that the maximum force a subject can exert declines as the muscle capacity diminishes because of fatigue (Ma et al., 2009). With this assumption, the endurance time decreases as more batches are processed. Therefore, MET for a given batch varies between two extreme values, which are when the body is fresh or completely recovered from previously accumulated fatigue and when the body is in the state of complete tiredness. Thus, and for simplicity, it is assumed that $f_{M V C}=i \lambda$, in which $i$ is the batch number and $\lambda$ is the fatigue index. Therefore $f_{M V C}$ is equal to $\lambda$ for the first batch, $2 \lambda$ for the second batch and so on. Note that one may represent $f_{M V C}$ as a function of time, $f_{M V C}(t)=\lambda(t)$; however, lack of evidence and data, and without loss of generality, it was assumed that $f_{M V C}=i \lambda$, where $i$ in the batch number that increases with time. More empirical research is still needed in this regard.

\subsection{The Learning Forgetting Fatigue Recovery Model (LFFRM)}

The LFCM in Section 4.2 favours a policy of longer production cycles with short breaks as this improves productivity. However, an ergonomic policy, aimed at minimizing injury risks, will be to minimize a worker's fatigue through short production cycles and rest breaks of lengths that guarantee quick recovery. Here, the LFFRM is a constrained LFCM model. The production time in cycle $i$ cannot exceed MET. From (4.1) we have: 
$t_{x_{i}}=\int_{u_{i}}^{x_{i}+u_{i}} T_{x} d x=\int_{u_{i}}^{x_{i}+u_{i}} T_{1} x^{-b} d x=\frac{T_{1}}{1-b}\left[\left(x_{i}+u_{i}\right)^{1-b}-u_{i}^{1-b}\right]$

where $t_{x_{i}}$ is the production time in cycle $i$ which should be equal or less than MET:

$t_{x_{i}} \leq M E T$

from which the following constraint is determined:

$0<x_{i} \leq\left(\frac{1-b}{T_{1}} \beta_{0} e^{-\beta \cdot f_{\mathrm{MVC}}}+u_{i}^{1-b}\right)^{1 /(1-b)}-u_{i} \quad$ or $\quad 0<x_{i} \leq x_{i}^{U}$

where $x_{i}^{U}$ is the upper bound of units that can be produced in batch $i$. Ideally, the length of a rest break would not exceed the time for total forgetting, i.e., $\tau_{i} \leq B$. However, the break time could either be the time a worker spends performing another task (producing a different product) during which some of the strained body muscles are relaxed and recovered or, it could be a real rest break. Also one might assume that at the end of the shift the person is largely "on break" until work starts - so long as they are not doing the same kind of tasks at home or off hours. The length of a work break is usually governed by output constraints that managers have to weigh carefully against the workers' welfare and the system's productivity. Therefore, the length of a break should be less than $B$, but long enough to alleviate a significant amount of the worker's fatigue. It is assumed that management would always try to balance between the workers' welfare and system's productivity. Therefore, management would like to alleviate at least $\delta \%$ of 
a worker's accumulated fatigue and ensure that $\theta \%$ of the experience is transferred from one batch to the next. If a manager wants to alleviate $\delta \%$ of the worker's fatigue then from (4.8), $R\left(\tau_{i}\right)=\delta \times F(t)=F(t) e^{-\mu \tau_{i}}$, implying that $\tau_{i} \geq-\ln (\delta) / \mu$. On the other hand, a manager has to sustain an acceptable level of productivity by retaining a specific level of learning to be transferred between cycles, e.g., at least $\theta \%$ of the worker's experience should be retained, then from (4.4), (4.5) and (4.8), we have:

$-\ln (\delta) / \mu \leq \tau_{i} \leq \frac{T_{1}}{1-b}\left\{\left[\frac{\theta}{\left(u_{i}+x_{i}\right)^{f_{i} / b}}\right]^{-b(1-b) / f_{i}}-\left(u_{i}+x_{i}\right)^{1-b}\right\}, \quad$ or $\quad \tau_{i}^{L} \leq \tau_{i} \leq \tau_{i}^{U}$

where $\tau_{i}^{L}$ is the lower bound and $\tau_{i}^{U}$ is the upper bound of the break length, and $\theta$ is the specific level of learning transferred between cycles. The derivation of (4.14) is shown in Appendix I.

The average processing time (performance) $Z$ per unit, is computed by dividing the total processing time by the total number of units produced as:

$$
Z=\frac{\sum_{i=1}^{N} t_{x_{i}}}{\sum_{i=1}^{N} x_{i}}
$$

where $N$ is the total number of batches. The productivity of the system is highest when (4.15) records its lowest value. Therefore, the above model could be written as Minimize $Z$; subject to the following constraints: (1) $0<x_{i} \leq x_{i}^{U}$, and (2) $\tau_{i}^{L} \leq \tau_{i} \leq \tau_{i}^{U}$. 


\subsection{Methodology}

The behaviour of the Learning Forgetting Fatigue Recovery Model (LFFRM) is now investigated using several numerical examples to demonstrate how the input parameters, i.e., learning index, number of batches, fatigue index, and recovery index, influence the outcomes of the production process. The numerical results are discussed to highlight the managerial implications and insights of the developed model. For this purpose, the results are those of the optimal solutions. That is, the optimal solution is determined by minimising $Z$ in (4.15), subject to the constraints of (4.13), $0<x_{i} \leq x_{i}^{U}$, and (4.14), $\tau_{i}^{L} \leq \tau_{i} \leq \tau_{i}^{U}$, where $i=1, \ldots, N$. The input and output parameters are as follow:

The input parameters:

- The learning index is set to 3 levels corresponding to the slow (90\%), moderate (80\%), and fast (70\%) learning with $b=0.152, b=0.322$ and $b=0.515$, respectively (Dar-El et al., 1995).

- The parameters for $M E T$ calculation, $\beta_{0}=4.16$ and $\beta=7.96$, are taken from the study of Rose et al. (1992).

- Fatigue alleviation factor $(\delta)$ and the experience transfer factor $(\theta)$ are set at $50 \%, \delta=\theta=$ 0.5 .

- The time required to produce the first item is set to 1 unit of time, $T_{1}=1$.

- Total forgetting time is set to $B=2000$ units of time.

- The fatigue index is set to 3 levels corresponding to the slow, moderate, and fast fatigue accumulation with $\lambda=0.01, \lambda=0.03$ and $\lambda=0.05$, respectively. 
- The recovery index is set to 3 levels corresponding to the slow, moderate, and fast recovery with $\mu=0.03, \mu=0.05$ and $\mu=0.07$ respectively.

The last two parameters, fatigue accumulation index and recovery index, were determined using a test. With the assumption of $80 \%$ maximum fatigue per unit of time, the fatigue index was obtained from (4.7) as 1.6094. With the assumption of $80 \%$ recovery, the recovery index per unit of break time is also obtained from (4.8) as 1.6094 . The optimal problem was examined with these measures but no feasible solution was obtained. These values decreased until the above set of $\lambda$ and $\mu$ values was found that yield optimal solutions.

The optimization outputs:

- Z: Average unit processing time, which is computed from equation (4.15), is the performance measure of the system.

- F: Average accumulated fatigue from production of $N$ batches, which is computed from equations (4.7) - (4.9).

- $Q$ : Production volume as restricted by (4.13) where $Q=\sum_{i=1}^{N} x_{i}$.

- $\tau$ : Average length of a rest break between batches, where the number of breaks is equal to the number of batches minus one, is computed as $\tau=\sum_{i=1}^{N} \tau_{i}$, which is restricted by (4.14). 


\subsection{Results}

\subsubsection{What effect does the learning index have on the production process?}

In order to investigate the behaviour of the process regarding the learning index, in addition to the input parameters and their values given in Section $4.5\left(T_{1}=1, B=2000, \theta=50 \%\right.$, and $\delta$ $=50 \%$ ), the following input parameters are assumed: (1) Three learning indices are selected as discussed in 4.5, (2) fatigue and recovery indices are set to medium levels, i.e., $\lambda=0.03$ and $\mu$ $=0.05$, and (3) ten batches, $N=10$, are considered for the process. The mathematical programming model described by equations (4.13), (4.14) and (4.15) is optimised for these input parameters. The optimal average length of a beak in-between adjacent batches for the three learning indices was found to be $\tau^{*}=6.02$. The reason that the optimum break time is not changed with the learning is because it depends on $\delta$, the fatigue alleviation factor, and on $\mu$, the recovery index, as per (4.14), which restricts the break length to a value irrespective of the learning rate. Figure 4.3 shows that, as learning becomes faster $(b=0.515)$, the system's performance, $Z$, improves and becomes flatter. This suggests a range of values over which $Z$ is relatively stable which may provide managers with more flexibility to meet their obligations for their workers' welfare while also meeting their productivity targets. Other results are summarised in Table 4.2 . 


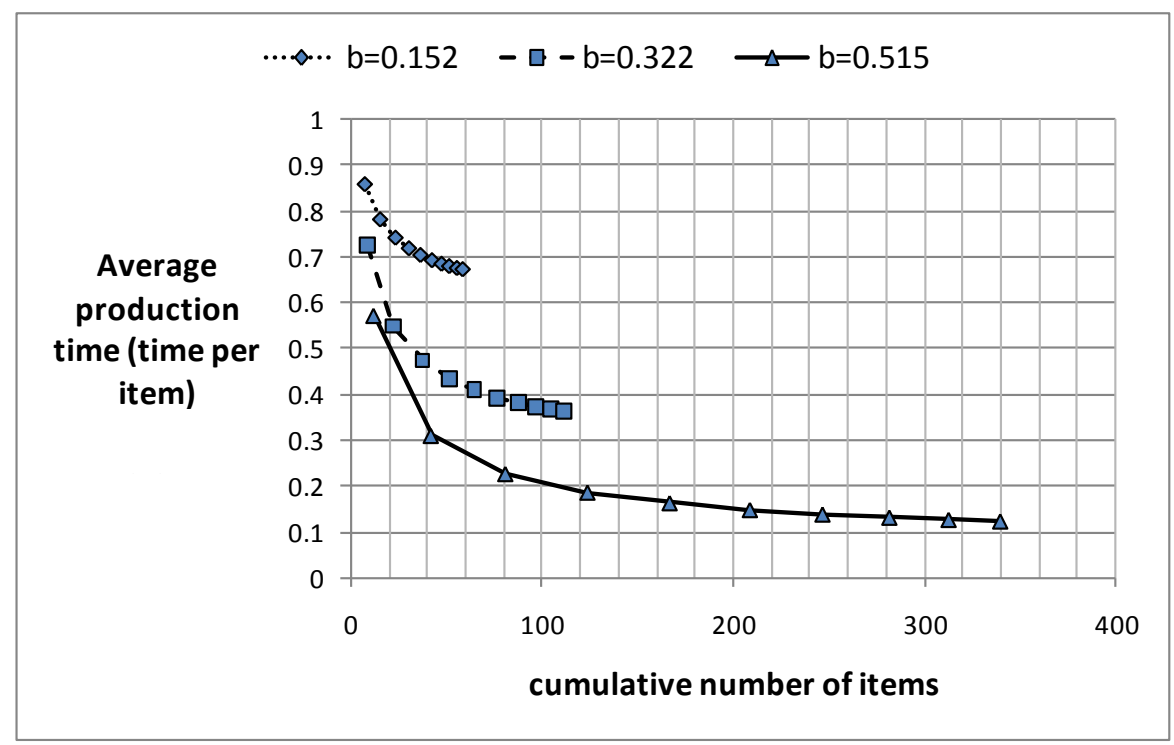

Figure 4.3. The behaviour of the systems optimal performance for different learning indices.

Table 4.2. The effect of learning index on the process outputs when $\lambda=0.03$ and $\mu=0.05$.

\begin{tabular}{|l|c|c|c|}
\hline Learning indices & $\boldsymbol{b}=\mathbf{0 . 1 5 2}$ & $\boldsymbol{b}=\mathbf{0 . 3 2 2}$ & $\boldsymbol{b}=\mathbf{0 . 5 1 5}$ \\
\hline Production volume, $Q$ & 59 & 112 & 340 \\
\hline Total production time, $\sum_{i=1}^{N} t_{x_{i}}$ & 39.64 & 40.82 & 42.19 \\
\hline Average fatigue (\%) & 17.44 & 17.87 & 18.39 \\
\hline Average production time per unit, $Z$ & 0.67 & 0.36 & 0.12 \\
\hline
\end{tabular}

The results in Table 4.2 show that as learning becomes faster ( $b$ increases from 0.152 to 0.515 ), the production volume increases by about $82 \%$ (from 59 to 340). The average production time per unit, or $Z$, decreases (improves) correspondingly from 0.67 to 0.12 as shown in Figure 4.3 but average fatigue does not change considerably with the learning. The results also suggest that a worker with fast learning experiences slower fatigue accumulation and improves the system's performance (smaller $Z$ values). This further suggests that investing to improve workers learning may be a good strategy for processes that are highly labour intensive. 
It should be noted that the actual performance of the system does not represent a smooth learning curve. Learning data usually shows some variations with every repetition (unit produced). However, the variation in performance between subsequent repetitions tends to reduce as the number of repetition increases. To capture the variation in performance, upper and lower bounds of a learning curve are set (Jaber, 2006).

\subsubsection{What effects does the number of batches have on the production process?}

In order to investigate the behaviour of the process with the number of batches, in addition to the input parameters and their values given in $4.5\left(T_{1}=1, B=2000, \theta=50 \%\right.$, and $\left.\delta=50 \%\right)$, the following input parameters are assumed: (1) The learning, fatigue, and recovery indices are set at their medium values as $b=0.322, \lambda=0.03$, and $\mu=0.05$, respectively, and (2) two batch numbers are considered, which are $N=5$ and $N=10$. The mathematical programming model described by (4.13), (4.14), and (4.15) is optimised for these input parameters. The results in Table 4.3 show that the production volume and subsequently the total production time increase as the number of batches $N$ increases from 5 to 10 . The optimal break length for both plans was found to be $\tau^{*}=6.02$. However, the average fatigue decreases by about $16 \%$ and the average performance ( $Z$ ) improves by about $12 \%$ with more batches, i.e. $N=10$. It may be concluded from these results that producing in more batches positively impacts $Z$ and improves workers' welfare as more rest breaks provided reduce the average fatigue level. It should be noted that the length of the break is the outcome of the program optimization and the result of aiming the target of maximum production, which relates to the worker's fatigue in LFFRM. Therefore, the break length may not be considered as reasonable comparing to the production time. These results are indicators of the extreme cases of optimality and dependant on values of the input parameters. Having said that, 
more reasonable break times may be obtained by introducing real data to the LFFRM model collected from actual industrial setting.

Table 4.3. The effect of the number of batches on the production process when $b=0.322, \lambda=0.03$, and $\mu=0.05$.

\begin{tabular}{|l|c|c|}
\hline No. of Batches & $\boldsymbol{N}=\mathbf{5}$ & $\boldsymbol{N}=\mathbf{1 0}$ \\
\hline Production volume, $Q$ & 65 & 112 \\
\hline Total production time, $\sum_{i=1}^{N} t_{x_{i}}$ & 26.74 & 40.81 \\
\hline Average length of the rest break, $\tau$ & 6.02 & 6.02 \\
\hline Average fatigue (\%) & 21.26 & 17.87 \\
\hline Average production time per unit, $Z$ & 0.41 & 0.36 \\
\hline
\end{tabular}

\subsubsection{What effects does the fatigue index have on the production process?}

In order to investigate the behaviour of the model regarding the fatigue accumulation index, in addition to the input parameters and their values as set in Section 4.5 ( $T_{1}=1, B=2000, \theta=50 \%$, and $\delta=50 \%$ ), the following input parameters are assumed: (1) Three different fatigue indices are selected for slow $(\lambda=0.01)$, medium $(\lambda=0.03)$ and fast $(\lambda=0.05)$ fatigue accumulation, (2) learning and recovery indices are set at their medium values as $b=0.322$ and $\mu=0.05$, and (3) ten batches, $N=10$, are considered for the process. The mathematical programming model described by (4.13), (4.14), and (4.15) is optimised for these input parameters. The results are summarised in Table 4.4 . 
Table 4.4. The effect of fatigue index on the production process when $b=0.322$ and $\mu=0.05$.

\begin{tabular}{|l|c|c|c|}
\hline Fatigue index & $\lambda=\mathbf{0 . 0 1}$ & $\lambda=\mathbf{0 . 0 3}$ & $\lambda=\mathbf{0 . 0 5}$ \\
\hline Production volume, $Q$ & 209 & 112 & 65 \\
\hline Total production time, $\sum_{i=1}^{N} t_{x_{i}}$ & 62 & 40 & 28 \\
\hline Average fatigue (\%) & 9 & 17 & 20 \\
\hline Average production time per unit, $Z$ & 0.3 & 0.36 & 0.43 \\
\hline
\end{tabular}

The results show that the fatigue index has a very little effect on the behaviour of the system performance $(Z)$. This is because the numerator and denominator of (4.15) both decline with more fatigue. The optimum length of the rest break was found to be $\tau^{*}=6.02$ for all cases. Table 4.4 illustrates that with faster fatigue accumulation (from $9 \%$ to $20 \%$ ), the production volume $Q$ decreases by $68 \%$ which suggests that the production volume actually declines as a direct result of worker fatigue. Figure 4.4 suggests that physically difficult tasks (larger $\lambda$ value) negatively affect the production output (cumulative number of items produced reduces) because a fatigued worker tends to produce less, either in smaller batches or shorter production runs.

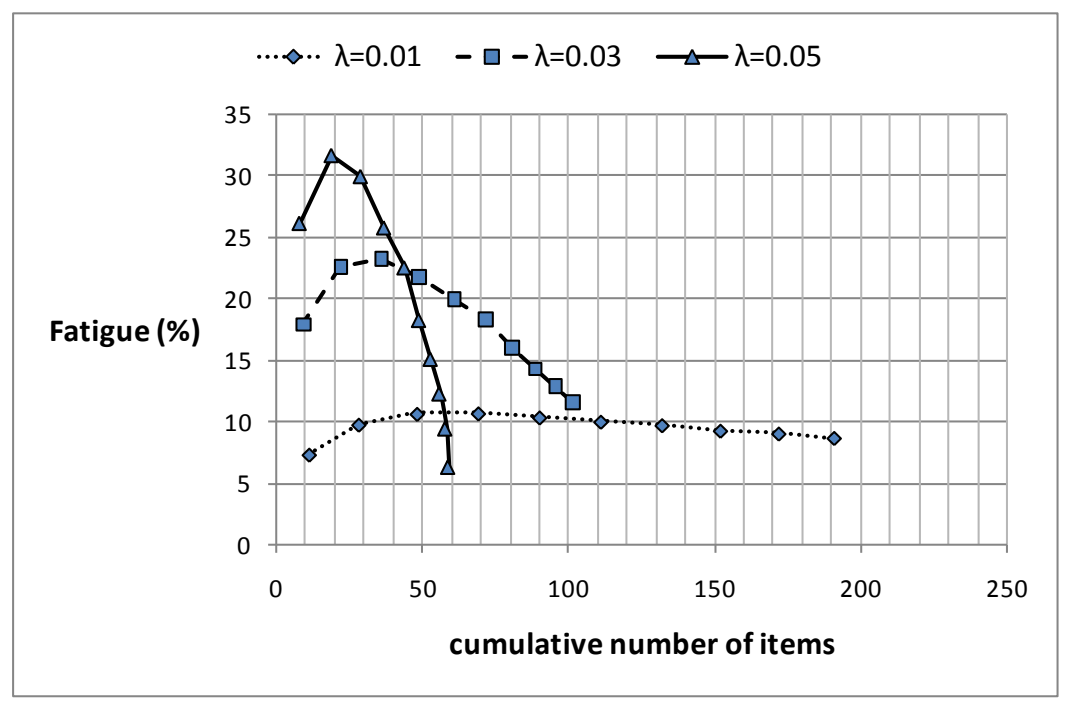

Figure 4.4. The behaviour of the fatigue curve for different $\lambda$ values representing different levels of task difficulty. Production volume declines as the task difficulty increases. 


\subsubsection{What effects does the recovery index have on the production process?}

In order to investigate the behaviour of the process regarding the recovery index, in addition to the input parameters and their values given in section $4.5\left(T_{1}=1, B=2000, \theta=50 \%\right.$, and $\left.\delta=50 \%\right)$, the following input parameters are assumed: (1) Three different recovery indices are selected for slow ( $\mu=0.03)$, medium $(\mu=0.05)$ and fast $(\mu=0.07)$ recovery, (2) learning and fatigue indices are set at their medium values as $b=0.322$ and $\lambda=0.05$, respectively, and (3) ten batches, $N=10$, are considered for the production process. The mathematical programming model described by (4.13), (4.14), and (4.15) is optimised for these input parameters. The results are summarised in Table 4.5, which show that as recovery from fatigue becomes faster, $\mu$ increases from 0.03 to 0.07 , production volume $Q$ increases from 102 to 118 and the total production time decreases from 130 to 79 resulting in an improvement in the system performance $Z$ of about $13 \%$ (from 0.4 to 0.35 ), and reducing the length of a rest break by $60 \%$ (from 10 to 4 ).

Table 4.5. The effect of recovery index on the production process when $b=0.322$ and $\lambda=0.03$.

\begin{tabular}{|l|c|c|c|}
\hline Recovery index & $\mu=\mathbf{0 . 0 3}$ & $\mu=\mathbf{0 . 0 5}$ & $\mu=\mathbf{0 . 0 7}$ \\
\hline Production volume, $Q$ & 102 & 112 & 118 \\
\hline Total production time, $\sum_{i=1}^{N} t_{x_{i}}$ & 130 & 95 & 79 \\
\hline Average length of the rest break, $\tau$ & 10 & 6.02 & 4 \\
\hline Average production time per unit, $Z$ & 0.4 & 0.36 & 0.35 \\
\hline
\end{tabular}

Lead time is defined as the sum of the production times for all 10 batches and the lengths of the 9 production breaks. As recovery becomes faster, the length of the production breaks are affected considerably. For a slow recovery, i.e. $\mu=0.03$, the optimal break length obtained as $\tau^{*}=10$ 
units of time, for a medium recovery, $\mu=0.05, \tau^{*}=6.02$, and for a fast recovery, $\mu=0.07$, $\tau^{*}=4$, which results in about $47 \%$ reduction in the lead time in the fast recovery case. The average production time is not changing considerably with the rate of recovery since in this case the production is not constrained by the fatigue level. The results of Table 4.5 indicate an increase in the lead time due to slow recovery. It may be concluded from the results that according to the LFFRM model, slow recovery deteriorates the process lead time which has not been considered in the unit production time, $Z$.

\subsection{LFFRM constraints and feasible solutions}

This section elaborates the importance of the constraints of the LFFRM model. We may find solutions for the LFFRM model without the constraints, but they are not feasible solutions. The solutions of an optimization problem usually lie in a more restricted range regarding to the general solutions. By studying the constraints of the LFFRM model, we will test the solutions and their feasibility. More specifically, we need to demonstrate how much the results would differ if the constraints are not considered and how this impacts the problem. The LFFRM constraints arise from a worker maximum endurance time (upper limit of production in (4.13)), the necessary level for fatigue alleviation (lower limit of break length in (4.14)) and maximum forgetting (upper limit of the break time in (4.14)).

In order to study the effects of the constraints on the model, in addition to the input parameters given in section $4.5\left(T_{1}=1, B=2000, \theta=50 \%\right.$, and $\left.\delta=50 \%\right)$, other parameters are set as follows:

(1) Two batch numbers are considered, $N=5$ and $N=10$, (2) total production volume is set to $Q$ $=200$ items, i.e., 200 items are produced in 5 or 10 batches with $x_{i}=20$ and $x_{i}=40$ items per 
batch, respectively, and (3) three break times of $\tau=1.5, \tau=6$, and $\tau=12$ are considered for the production process. Equations (4.1) to (4.15) are solved without considering constraints (4.13) and (4.14). Further, the effects of learning, fatigue accumulation, and recovery rates on the outputs were studied. When studying the effect of one parameter, we set the other parameters to their medium values. Results are in accordance with our previous observations indicating that fatigue is reduced when: learning is fast $(b=0.515)$, production occurs in small more frequent batches $(N=10)$, break times are longer $(\tau=12)$, and there is faster recovery from fatigue ( $\mu=0.07$ ). This indicates that although some of the solutions produced when the constraints are ignored are infeasible, the model's behaviour remains the same as when the constraints are imposed.

To consider the limitations of fatigue allowances which set the upper and lower bounds on the production quantity produced in each batch and the length of the rest, we consider the case where $b=0.152$ (slow learning), $\lambda=0.03$ (medium fatigue accumulation), and $\mu=0.05$ (medium recovery). The optimal solution occurs when $Q=37$ units and four rest breaks, each of $\tau=6$ units of time are provided result to $F=20.8 \%$, and $Z=0.76$. Excluding the constraints in (4.13) and (4.14) we have $Q=200$ units, four rest breaks of $\tau=1.5$ units of time each, $F=64.6 \%$, and $Z=0.586$. Comparing these cases, it is clear that solving the problem without the fatigue constraint, will yield better results regarding the production, i.e., more production $(Q \uparrow)$ in less time $(Z \downarrow)$, but poor worker welfare as average fatigue increases $(F \uparrow)$, i.e., $64.6 \%$ versus $20.8 \%$. Furthermore, the optimization regarding the required alleviating of $50 \%$ of the worker's fatigue will yield a rest break of $\tau=6$ units of time in length $(\tau \uparrow)$. This further means that having $\tau=1.5$ units of time is not possible unless workers welfare is ignored. 


\subsection{Discussion and conclusion}

In this Phase of the thesis, a Learning Forgetting Fatigue Recovery Model (LFFRM) has been developed and exemplified through numerical examples. Our contribution was to develop a model which integrates the "Learn Forget Curve Model" (LFCM) with a fatigue-recovery model since these two phenomena occur simultaneously during the production process and production breaks. This model is capable of capturing the quantitative effects of workers' abilities, i.e., improvement through learning and replenishment of the resources by recovery, and restrictions, i.e., losing experience by forgetting and capabilities due to physical fatigue, on the outputs of a production process. An interesting aspect of this research is that learning and recovery and forgetting and fatigue have opposite impacts on the performance, which requires trade-offs to optimise the system performance.

The effect of learning on the model has then been investigated and it was shown that, as learning becomes faster, the system performance improves, which is consistent with the previous studies (Benkard, 2000; Syverson, 2010). A further insight from the LFFRM model was that smaller batch sizes are recommended as they result in less fatigue. The benefits of reduced fatigue observed during the fast learning scenarios suggest that technologies and/or training programs may quicken the learning process of the workers. It has been shown that more production results in longer production times and, subsequently, more fatigue. In the LFFRM model the lengths of breaks are optimized such that enough fatigue is alleviated and not much experience is lost because of forgetting. Similar to previous studies, e.g. Tucker (2003), the model recommends shorter frequent breaks to increase the production output.

LFFRM model demonstrated that: 
i) Faster learning helps to reduce fatigue. Learning will cause the process to have more production with the same amount of fatigue. It may suggest to managers to consider investing in training programs as this meets two managerial objectives, the welfare of the workers and the productivity of the system.

ii) Fatigued workers are counterproductive. This finding has been observed by Mital et al. (1991) who showed that the worker performance declines because of fatigue. Fatigued workers also have higher likelihood of making errors and being injured (Kopardekar \& Mital, 1994; Dinges, 1995; Frank, 2000; Dionisio, 2010; Asfaw et al., 2011; Bevilacqua et al., 2012). Therefore direct and indirect results of fatigue may cause the process to be more costly (Ricci et al., 2007). If the length of a break is not enough to alleviate the fatigue, or if the fatigue rate is high, management could consider to invest in learning to lessen the fatigue, designing less fatiguing jobs, or allowing for longer breaks. This suggests that investing in training may be an improvement strategy for processes that are highly labour demanding either by training the workers, or by designing work tasks in ways that foster learning. For instance, designing material kits that are implicit instructive for assembly sequence (Medbo, 2003), encoding inferences into routines, e.g., forms, rules, procedures and strategies (Levitt \& March, 1988), adopting new technologies (Shafer et al., 2001), changing the product design, tools, equipment, and works methods (Konz \& Johnson, 2000), using knowledge diversity (Kellogg, 2009), and using cross-trained employees at numerous work stations (Finch \& Luebbe, 1995) have been mentioned as ways to speed up the learning. Another way to confront fatigue is to reduce the fatigue accumulation rate by controlling the workload levels for operators through reduced physical forces, improved 
worker postures, and reduced durations of force application (James \& William, 1993; Yeow \& Nath Sen, 2003).

iii) As the recovery becomes faster, the limitation on batch number is relieved and more units can be produced with less average production time (better performance). As a result, when the worker recovers faster from the accumulated fatigue, lead time shrinks. Recovery rate may be considered as a personal attribute. The author was not able to find any industry related study for speed up the recovery. However, there is literature available in the Physiology field, e.g. the study of Tessitore et al. (2007), which suggests aerobic exercises and electrostimulation help soccer players to recover faster and to avoid damage of their muscles.

In order to study the limitations that arise from worker's capabilities, the constraints of the LFFRM model were studied. The LFFRM constraints arise from the worker's maximum endurance time (fatigue), the necessary level for fatigue alleviation and maximum forgetting. Relieving the upper bound of the production volume constraint and lower bound of the break time constraint revealed that the real production process would not achieve the initial goals that have been set for it because of these constraints. The literature of the productivity loss due to health problems (Punnett \& Wegman, 2004; Meerding et al., 2005; Escorpizo, 2008) may support the results of the LFFRM modelling. Worker's capabilities and limitations are crucial issues that must be considered in designing the future manufacturing systems (Keyserling \& Chaffin, 1986). 
The LFFRM of Phase 1 of this thesis, provides a basis for further research into the process improvement and also workforce scheduling however it has some limitations. First of all, only physical fatigue has been modeled in this version of LFFRM. Secondly, only the effect of fatigue on the productivity was investigated. Other aspects of the problem such as the effect of fatigue on the product quality have not been studied. Thirdly, the product variety has not been investigated and the process was assumed to produce only one type of product. Finally, I did not provide solutions for improving the learning and recovery or decreasing the fatigue accumulation; however, some solutions have been provided in the literature as discussed above. To refine the LFFRM model it would be best in the future to collect real-life data from manufacturing environment. This data will help to refine or modify the models and utilize them for optimization purposes in industry. 


\section{Phase 2: Incorporating forgetting into the quality learning curve, Quality Learning Forgetting Curve (QLFC)}

Phase 2 of the thesis investigates the effect of forgetting on a production process where some produced items do not conform to quality. Such items are considered to be defective and are then reworked. In this Phase, the forgetting effects are integrated into a known quality learning curve to develop the Quality Learning Forgetting Curve (QLFC). The results of this Phase are useful in production planning and the scheduling of workers in DRC systems in Phase 4.

\subsection{Introduction}

The main assumption of the Wright's learning curve (WLC; 1936) is that all units of production have acceptable quality. However, when production increases, the probability of the process going out-of-control increases for several reasons and non-conforming quality items are generated as a result (Pal et al., 2013). Therefore, at the start of production, the process is assumed to be in a control state and that produced items conform to quality. After some time, the process may go out-of-control and start generating non-conforming or defective items that may be either repaired or scrapped (Sana, 2010). This situation has been observed in many process industries (Flapper et al., 2002; Meyr, 2004; El Saadany, 2009; Glock \& Jaber, 2013a; Roy et al., 2013) where the defective items are reworked. If the rework process is also imperfect, double defective items are scrapped (Jamal et al., 2004; Peter Chiu et al., 2010; Taleizadeh et al., 2013).

Learning has been observed in production and rework processes where the cost of producing and repairing items is reduced as a result of using better tools and processes (Burr \& Pearne, 2013). Learning reduces production costs and cycle times (Sebrina \& Diawati, 2012; Tirkel, 2013) and inventories (Jaber et al., 2009), and improves the quality of manufactured products (Levin, 2000; 
Choo et al., 2007). Forgetting, on the other hand, is pertinent to deteriorating the quality and production levels and increasing the costs and inventories (Jaber et al., 2009; Teyarachakul et al., 2014). Failure to achieve the expected level of productivity makes a firm less competitive. Readers may refer to Jaber $(2006,2014)$ for a concise review on learning curves and their applications.

The research on how learning and quality interact interested many researchers, of which few are surveyed here. Koulamas (1992) found that there is a relationship between quality improvement activities such as product redesign and process performance. He also found that product redesign improves the quality of the products by decreasing the quality related problems but it increases the costs. Badiru (1995) investigated the effects of learning and forgetting on product quality and demonstrated that the actual unit cost is underestimated if forgetting effects are not considered. Jaber and Bonney (2003) further analysed the data of Badiru (1995) and empirically validated two hypotheses: the time required to rework a defective item declines following a learning curve, and the quality of the products deteriorates as the forgetting increases. In another study, Jaber et al. (2010) investigated a supply chain in which the manufacturer experiences a continuous improvement process through capacity utilization, reduction in the set-up times, and elimination of the defective items by rework. Their results justified a production policy with smaller lots and more frequent production and indicated that forgetting increases the costs throughout the supply chain. Readers are referred to Jaber and Bonney (2011) for a review of the studies that investigated the learning effects in lot sizing.

Research on the learning-quality relationship also shows that the improvement in the process quality follows a learning curve (Lapré et al., 2000) and targeting for higher levels of quality, 
moves organizations through steeper learning curves (Subedi, 2006). Stratman et al. (2004) investigated deployment policies regarding the hidden costs associated with defective items resulting from different levels of learning (skills) and forgetting. They found that assigning skilled workers to upstream operations is superior to the other policies. Readers are referred to Khan et al. (2011) for a good review of the studies that investigated the learning - quality relationship.

There is a debate in the literature about whether the learning curve improvement continues indefinitely (Zangwill \& Kantor, 1998; Ferioli et al., 2009) or if it ceases at a plateau for some of the following reasons: management unwillingness to invest, labour intentions for learning, depreciation in the knowledge, or forgetting (Kennedy \& Trafton, 2007; Peterson \& Jun, 2007; Feldman et al., 2009; Sikström \& Jaber, 2012; Takahashi, 2013; Tirkel, 2013). Jaber and Guiffrida (2004) argued that the plateauing may be attributed to quality problems and could be a barrier for process improvements.

An interesting model developed by Jaber and Guiffrida (2004) is the Quality Learning Curve (QLC) for a production process that generates defective items requiring rework. The QLC is a composite learning curve, which is the sum of two learning curves: one represents production while the other represents rework. They showed that the QLC may be of a convex form under some conditions. The convexity corresponds to a unique minimiser that reciprocates the best performance on the curve. Jaber and Guiffrida (2004) used the model of Porteus (1986) to calculate the number of defective items in a lot that requires rework. Like Porteus (1986), they assumed that once the process goes out of control it remains in that state until the entire lot is produced. In a follow up study, Jaber and Guiffrida (2008) revised their earlier work by allowing 
for the interruption of the production process to restore its quality, a notion adopted from Khouja (2005). The works of Jaber and Guiffrida $(2004,2008)$ have a major limitation. They assumed a single production cycle, the initial one. Jaber and Khan (2010) investigated the model of Jaber and Guiffrida (2004) in a serial production line with lot splitting where scrapped items at each stage are discarded. Jaber and Khan (2010) found that the system deteriorates as the number of stages in a serial production line increases. Like Jaber and Guiffrida (2004, 2008), Jaber and Khan (2010) considered no transfer of learning between subsequent batches.

None of the works surveyed above considered forgetting effects. Forgetting hinders the learning process when production is intermittent by breaks. After producing a lot, the production process ceases to allow the reworking of defective items and to release the process for other tasks. In this situation, workers forget some of the knowledge previously acquired because of alternating between different tasks (Jaber et al., 2003).

Psychologists have reported that forgetting may occur in any of the following situations: (1) when there is not enough similarity between the conditions of encoding and retention of material learned, (2) when old learning interferes with new learning, and (3) when there is an interruption in the learning process for a period of time (production break) (Jaber, 2014, p.549). Knowledge depreciation could also occur due to worker turnover, changes in a product or a process, or loss of organizational records (Jaber, 2014). Knowledge depreciation was reported in energy technology, aircraft production, automotive, textile and information technology industries, shipyards, and fast food franchises (Nembhard, 2000; Kim \& Seo, 2009; Argote, 2013; Kang \& Hur, 2013; Grubler \& Nemet, 2014). 
Phase 2 of this research extends the work of Jaber and Guiffrida (2004, 2008) by assuming forgetting to occur when a worker alternates between the production and rework segments of a cycle and when cycles are interrupted by production breaks. It aims to incorporate the aspects of production quality into the learning and forgetting curve and demonstrate the effect of forgetting on the production planning in this perspective. The developed model in Phase 2 is called Quality Learning Forgetting Curve (QLFC) and it will be used in developing the production planning in DRC systems in Section 7.

The rest of Section 5 is organized as follows: Section 5.2 provides a review of the QLC model, the methodology of Phase 2 is demonstrated in Section 5.3. Section 5.4 provides the numerical examples. Results are presented in Section 5.5 and discussed in Section 5.6.

\subsection{Review of the Quality Learning Curve (QLC)}

This section provides a brief background to the mathematics of the Quality Learning Curve (QLC) of Jaber and Guiffrida (2004) and Jaber and Guiffrida (2008). Jaber and Guiffrida (2004) considered two QLC cases. The first case assumed learning in production but no learning in the rework, while the second case assumed learning in both production and rework.

The learning curve for the first case was found to be of a convex form with an optimum lot size that has the best performance on the curve, of:

$$
x^{* *}=\left(\frac{T_{1} b}{r_{1} \rho}\right)^{\frac{1}{1+b}}
$$


The learning curve for the second case was also found to be of a convex form, only if $0.0 \leq \varepsilon<0.5$, with an optimum lot size of:

$x^{* *}=\left(\frac{T_{1} b}{2 r_{1}(1-2 \varepsilon)(\rho / 2)^{1-\varepsilon}}\right)^{\frac{1}{1+b-2 \varepsilon}}$

The QLC was shown to plateau when $\varepsilon=0.5$ and to continuously improve for any $0.5 \leq \varepsilon<1$, behaving similar to the Wright's learning curve.

In a follow-up study, Jaber and Guiffrida (2008) assumed that the production process can be interrupted and restored back to the in-control state, with the restoration time being a percentage of the production time. Similar to their earlier work, they also considered two cases. The learning curve for the first case was found to be a convex form with an optimum lot size of:

$x^{* *}=\left(\frac{T_{1} b+T_{1} b \xi(1-1 / n)^{1-b}}{r_{1} \rho / n}\right)^{\frac{1}{1+b}}$

The learning curve for the second case was also found to be convex, only if $0.0 \leq \varepsilon<0.5$, with an optimum lot size of:

$x^{* *}=\left(\frac{T_{1} b+p_{1} b \xi(1-1 / n)^{1-b}}{2 r_{1}(1-2 \varepsilon)(\rho / 2 n)^{1-\varepsilon}}\right)^{\frac{1}{1+b-2 \varepsilon}}$ 
If the restoration time is negligible, i.e. $\xi=0$, as it is assumed in this section, then (5.3) and (5.4) will converge to $x^{* *}=\left(\frac{T_{1} b}{r_{1} \rho / n}\right)^{\frac{1}{1+b}}$ and $x^{* *}=\left(\frac{T_{1} b}{2 r_{1}(1-2 \varepsilon)(\rho / 2 n)^{1-\varepsilon}}\right)^{\frac{1}{1+b-2 \varepsilon}}$, respectively.

\subsection{Methodology}

In practice, production is intermittent. The break time between two production lots (or cycles) that allows for performing other tasks results in forgetting, where some of the experience (knowledge) gained from previous lots is lost. It is assumed that in each cycle a production run is followed by a rework run to repair some of the defective items generated during the production run. Like the work of Jaber and Guiffrida $(2004,2008)$ I also consider two cases: (1) learning in production but no learning in rework, and (2) learning in production and rework.

The following assumptions are made in this section:

- The rework process is always in control, and no scrap (double defective) item is generated.

- Once the process goes out of control with a given probability, defective items start accumulating.

- The time to restore the production process is negligible in comparison to the cycle time, i.e. production plus rework times.

\subsubsection{Learning in production and no learning in rework $(\varepsilon=0.0)$}

If $r$ is the time to rework a defective item, then $r d\left(x_{i}\right)$ is the time to rework $d\left(x_{i}\right)$ units. The number of defective items in a lot of size $x_{i}$ is estimated from Khouja (2005) as $d\left(x_{i}\right)=\rho x_{i}^{2} / 2 n$, where $\rho$ is the probability that the process goes out of control and produces defective items, $x_{i}$ is 
the lot size, and $n$ is the number of maintenance restorations in a lot, which is divided as a result into $n-1$ sublots. The total time to produce a lot is the sum of the production $\left(Y\left(x_{i}\right)\right)$ and rework time $\left(R\left(x_{i}\right)\right)$ in a serial line from LFCM model (section 4.2):

$T\left(x_{i}, n\right)=Y\left(x_{i}\right)+R\left(x_{i}\right)=\frac{T_{1}}{1-b}\left[\left(x_{i}+u_{i}\right)^{1-b}-u_{i}^{1-b}\right]+\frac{r \rho x_{i}^{2}}{2 n}$

where $u_{i}$ is experience (in units) transferred to production run $i$ from $i-1$ previous runs following an interruption. The time per unit, or the learning curve, is determined by differentiating (5.5) with respect to (w.r.t) $x_{i}$ to get:

$t\left(x_{i}, n\right)=y\left(x_{i}\right)+r\left(x_{i}\right)=T_{1}\left(x_{i}+u_{i}\right)^{-b}+\frac{r \rho x_{i}}{n}$

Lemma 1: The learning curve expression in (5.6) is convex in $x$ with a unique minimum at $x^{*}$.

Proof: The first and second derivatives of (5.6) w.r.t. $x$ are given, respectively, as:

$\partial t\left(x_{i}, n\right) / \partial x_{i}=-T_{1} b\left(x_{i}+u_{i}\right)^{-b-1}+\frac{r \rho}{n}>0 ; \forall x_{i} \geq 1$

and

$\partial^{2} t\left(x_{i}, n\right) / \partial x_{i}^{2}=T_{1} b(1+b)\left(x_{i}+u_{i}\right)^{-(b+2)}>0 ; \forall x_{i} \geq 1$

then (5.6) is convex and has a unique minimum, which is obtained by setting the first partial derivative of (5.6) equal to zero and solving for $x_{i}$ to get: 


$$
x_{i}^{*}=\left(\frac{T_{1} b n}{r_{1} \rho}\right)^{\frac{1}{1+b}}-u_{i}
$$

Note that for $n=1$ and $u_{i}=0,(5.9)$ reduces to (5.1). Also note that $x_{i}^{*}$ must be a feasible quantity with respect to production capacity of the process. The related numerical examples are provided in sections 5.4.1.1 and 5.4.2.1.

\subsubsection{Learning in production and rework $(\varepsilon \neq 0.0)$}

When learning in rework is considered, (5.5) can be written as:

$$
T\left(x_{i}\right)=Y\left(x_{i}\right)+R\left(x_{i}\right)=\frac{T_{1}}{1-b}\left[\left(x_{i}+u_{i}\right)^{1-b}-u_{i}^{1-b}\right]+\frac{r_{1}}{1-\varepsilon}\left[\left(d\left(x_{i}\right)+v_{i}\right)^{1-\varepsilon}-v_{i}^{1-\varepsilon}\right]
$$

where $r_{1}$ is the time to rework the first unit and $d\left(x_{i}\right)=\rho x_{i}^{2} / 2 n$ is the number of defective units that need reworking. The term $v_{i}$, similar to $u_{i}$, is the experience (in units) transferred to rework run $i$ from $i-1$ previous runs performed before the interruption occurs, while $\varepsilon$ is the learning index for the rework process. The time per unit or the learning curve is determined by differentiating (5.10) w.r.t $x_{i}$ to get:

$t\left(x_{i}\right)=y\left(x_{i}\right)+r\left(x_{i}\right)=T_{1}\left(x_{i}+u_{i}\right)^{-b}+\frac{r_{1} \rho x_{i}}{n}\left(\frac{\rho x_{i}^{2}}{2 n}+v_{i}\right)^{-\varepsilon}$

Note that, if $\varepsilon=0.0$ in (5.11), (5.6) is obtained. 
Equation (5.11) holds for every value of the rework learning index $\varepsilon$ and it is used in providing three lemmas. The rework learning index between 0.0 and $0.5 ; 0<\varepsilon<0.5$, is discussed in section 5.3.3 and lemma 2. The rework learning index $0.5, \varepsilon=0.5$, is discussed per section 5.3.4 and lemma 3 and 4. The rework learning index between 0.5 and $1,0.5<\varepsilon<1$, is discussed in section 5.3.5. No lemma is assigned to this case.

\subsubsection{Cases of $0.0<\varepsilon<0.5$}

Lemma 2: The learning curve expression in (5.11) is convex in $x_{i}$ with a unique minimum at $x_{i}^{*}$.

Proof: $y\left(x_{i}\right)$ in (5.11) is a monotonically decreasing function of $x_{i}$ since:

$$
\partial y / \partial x_{i}=-b T_{1}\left(x_{i}+u_{i}\right)^{-b-1}<0 ; \forall x_{i}>0
$$

and

$$
\partial^{2} y / \partial x_{i}^{2}=b(b+1) T_{1}\left(x_{i}+u_{i}\right)^{-b-2}>0 ; \forall x_{i}>0
$$

Similarly for $0<\varepsilon<0.5, r\left(x_{i}\right)$ in (5.11) is a monotonically increasing function of $x_{i}$. The first and second derivatives of $r\left(x_{i}\right)$ in (5.11) w.r.t. $x_{i}$ are given respectively as:

$\partial r / \partial x_{i}=r \rho n^{\varepsilon-1}\left(\frac{\rho x_{i}^{2}+2 v_{i} n}{n}\right)^{-\varepsilon-1}\left(\rho 2^{\varepsilon} x_{i}^{2}+2^{1+\varepsilon} v_{i} n-2^{1+\varepsilon} \rho x_{i}^{2} \varepsilon\right)>0 ; \forall x_{i}>0,0<\varepsilon<0.5$

and 
$\partial^{2} r / \partial x_{i}^{2}=r \varepsilon \rho^{2} n^{\varepsilon-1}\left(\frac{\rho x_{i}^{2}+2 v_{i} n}{n}\right)^{-\varepsilon-2}\left(2^{1+\varepsilon} \rho x_{i}^{2}+12 \times 2^{1+\varepsilon} v_{i} n-4 \times 2^{\varepsilon} \rho x_{i}^{2} \varepsilon\right)>0 ; \forall x_{i}>0,0<\varepsilon<0.5$

Therefore, the sum of monotonically decreasing, $y\left(x_{i}\right)$, and increasing, $r\left(x_{i}\right)$, functions as in (5.11), i.e. $t\left(x_{i}\right)$, is a convex function with a unique minimiser.

The optimum lot size that minimises (5.11) is found by equating the first derivative of (5.11) to zero as:

$\frac{\partial t\left(x_{i}\right)}{\partial x_{i}}=-T_{1} b\left(x_{i}+u_{i}\right)^{-b-1}+\frac{r_{1} \rho}{n}\left(\frac{\rho x_{i}^{2}}{2 n}+v_{i}\right)^{-\varepsilon}-\varepsilon \frac{r_{1} \rho^{2} x_{i}^{2}}{n^{2}}\left(\frac{\rho x_{i}^{2}}{2 n}+v_{i}\right)^{-\varepsilon-1}=0$

Equation (5.16) is solved numerically as no closed form solution exists. For the special case when $u_{i}=0$ and $v_{i}=0$, the solution for $x_{i}$ is obtained as:

$x_{i}^{*}=\left(\frac{r_{1} \rho(1-2 \varepsilon)}{n b T_{1}}\left(\frac{\rho}{2 n}\right)^{-\varepsilon}\right)^{\frac{1}{-1-b+2 \varepsilon}} ; 0<\varepsilon<0.5$

In (5.17), if $\varepsilon=0.0$, i.e. no learning in the rework process, and $n=1$, (5.17) reduces to (5.1). Also, if only $n=1$, it reduces to (5.2). Note that the unique minimiser of (5.11), $x_{i}{ }^{*}$, must be a feasible quantity with respect to production capacity of the process. The related numerical examples are provided in sections 5.4.1.2 and 5.4.2.2. 


\subsubsection{Cases of $\varepsilon=0.5$}

Lemma 3: With no forgetting in the production and rework process, and equal lots of size $x$ each, the learning process in (5.11) reaches a plateau when the rework learning index is onehalf, i.e, $\varepsilon=0.5$, and that the plateau value decreases as the number of restorations or interruptions increases.

Proof: For $\varepsilon=0.5$, in case of no forgetting in production and rework, the total experience accumulated from producing the first lot of size $x$ is transferred to the next lot of the same size $x$ reducing (5.11) to:

$t\left(x_{2}\right)=y\left(x_{2}\right)+r\left(x_{2}\right)=T_{1}\left(2^{1-b}-1\right) x^{-b}+r_{1}\left(2^{1 / 2}-1\right)\left(\frac{2 \rho}{n}\right)^{1 / 2} ;$ for the $2^{\text {nd }}$ lot

and it continues for $i$ cycles where:

$t\left(x_{i}\right)=y\left(x_{i}\right)+r\left(x_{i}\right)=T_{1}\left(i^{1-b}-(i-1)^{1-b}\right) x^{-b}+r_{1}\left(i^{1 / 2}-(i-1)^{1 / 2}\right)\left(\frac{2 \rho}{n}\right)^{1 / 2}$

When $x \rightarrow \infty$ (i.e. $x$ is a very large number), the first term $T_{1}\left(i^{1-b}-(i-1)^{1-b}\right) x^{-b} \rightarrow 0$ and the learning curve in (5.11) reaches the plateau value of $t\left(x_{i}\right) \rightarrow r_{1}\left(i^{1 / 2}-(i-1)^{1 / 2}\right)\left(\frac{2 \rho}{n}\right)^{1 / 2}$. The plateau term decreases as the number of splits, $n$, increases. The related numerical example is provided in section 5.4.1.3. 
Lemma 4: With forgetting in the production and rework process, the learning curve in (5.11) reaches a plateau when the rework learning index is one-half, i.e. $\varepsilon=0.5$, and that the plateau value decreases as the number of restorations or interruptions increases.

Proof: For $\varepsilon=0.5,(5.11)$ reduces to:

$t\left(x_{i}\right)=y\left(x_{i}\right)+r\left(x_{i}\right)=T_{1}\left(x_{i}+u_{i}\right)^{-b}+\frac{r_{1} \rho x_{i}}{n}\left(\frac{\rho x_{i}^{2}}{2 n}+v_{i}\right)^{-1 / 2}$

When $x_{i} \rightarrow \infty$ (i.e. $x$ is a very large number), the first term $T_{1}\left(x_{i}+u_{i}\right)^{-b} \rightarrow 0$, while the second term is written as:

$$
\frac{r_{1} \rho x_{i}}{n}\left(\frac{\rho x_{i}^{2}}{2 n}+v_{i}\right)^{-1 / 2}=\frac{r_{1} \rho x_{i}}{n \sqrt{\frac{\rho x_{i}^{2}}{2 n}+v_{i}}}=\frac{r_{1} \rho x_{i}}{n x_{i} \sqrt{\frac{\rho}{2 n}+\frac{v_{i}}{x_{i}^{2}}}}=\frac{r_{1} \rho}{n \sqrt{\frac{\rho}{2 n}+\frac{v_{i}}{x_{i}^{2}}}} ; \forall x_{i}>0
$$

As $x_{i} \rightarrow \infty$, the term $\left(\frac{v}{x_{i}^{2}}\right)$ becomes zero and the second term in (5.21) reduces to $r_{1}\left(2 \frac{\rho}{n}\right)^{1 / 2}$. Therefore, the learning curve in (5.20) approaches a plateau value; $t\left(x_{i}\right) \rightarrow r_{1}\left(2 \frac{\rho}{n}\right)^{1 / 2}$ as $x_{i} \rightarrow \infty$. When a lot of size $x_{i}$ is split into $n$ equal sublots, the plateau term decreases as the number of splits, $n$, increases. Investigation for very large numbers of cumulative production is important since learning asymptotically reaches a plateau suggesting no further improvement beyond that point. The plateau time as per (5.20) for $x_{i} \rightarrow \infty$ is used to estimate the standard (or steady state) time of the production (Teyarachakul et al., 2014). The same case was observed in the 
study of Jabar and Guiffrida (2004) where they suggested that plateauing might occur due to quality improvement efforts. The plateau phenomenon requires managers to invest in training or new technologies to be able to improve the production process. The related numerical example is provided in section 5.4.2.3.

\subsubsection{Cases of $0.5<\varepsilon<1$}

It can be easily shown that if $\varepsilon>0.5$, the derivative of equation (5.11) is negative for every $x_{i}$, i.e., $\frac{\partial t\left(x_{i}\right)}{\partial x_{i}}<0, \forall x_{i}$, which indicates that $t\left(x_{i}\right)$ is a continuously decreasing function. Therefore, for $0.5<\varepsilon<1$, the composite learning curve behaves similar to the Wright's learning curve with continuous improvement towards less times. The related numerical examples are provided in sections 5.4.1.4 and 5.4.2.4.

\subsection{Numerical examples}

For simplicity and feasibility reasons, only integer values are considered for lot sizes. In obtaining the numerical results, the values of the input parameters have been set according to Table 5.1.

Table 5.1. Parameters' initial values.

\begin{tabular}{lll}
\hline Description of the parameter & Symbol & Numerical values \\
\hline Production learning index & $b$ & $0.25,0.5,0.75$ \\
Rework learning index & $\varepsilon$ & $0,0.25,0.5$, and 0.75 \\
Time to produce the first unit & $T_{1}$ & 10 units of time \\
Time to rework the first unit & $r_{1}$ & 5 units of time \\
Probability to go out of control & $\rho$ & 0.001 \\
Total forgetting time & $B$ & 2000 units of time \\
\hline
\end{tabular}


The numerical examples are presented in two different cases for learning in production: (1) with no forgetting, where the production is performed in five lots with no loss of experience between lots, and (2) with forgetting, where the loss of experience occurs between subsequent lots. The first case, with no forgetting, is presented to compare its results with the previous works, and to set a benchmark for the second case, forgetting cases.

\subsubsection{No forgetting}

The no forgetting case is investigated for four different scenarios of learning in rework: (1) $\varepsilon=0.0$, (2) $0.0<\varepsilon=0.25<0.5$, (3) $\varepsilon=0.5$, and (4) $0.5<\varepsilon<1$.

\subsubsection{No learning in the rework process $(\varepsilon=0.0)$}

If there is no forgetting $(B \rightarrow \infty)$ in the production process, then any experience accumulated from producing $x$ units in each of $i$ lots is fully transferred to the next lot; i.e. $u_{i}=(i-1) x$. Then (5.9), can be rewritten as:

$$
x^{*}=\left(\frac{T_{1} n b\left(i^{1-b}-(i-1)^{1-b}\right)}{r_{1} \rho}\right)^{\frac{1}{1+b}}
$$

Using the values of parameters in Table 5.1 (except for $B$ ), for five consecutive lots, $i=5$, when $n=1,2$ or 3 , the results are summarized in Table 5.2 and Table 5.3. The results in Table 5.2 show that for any lot number 1 to 5 , the lot size decreases as learning becomes faster. For example, for two sublots, when $n=2, x^{*}$ decreases by $80 \%$ (from 185 to 37 ) when learning becomes faster ( $b$ increases from 0.25 to 0.75$)$. The reduction is about the same for $n=1(76 \%)$ 
and for $n=3(81 \%)$. These percentages slightly increase when the lot size number increases due to cumulative experience. The results also show that for a given learning index, say $b=0.25$, as the number of interruptions to restore the process increases, $n$ goes from 1 to $3, x^{*}$ increases. This is logical as less defective items are produced, allowing for more production and less rework. The results suggest that if a firm wishes to produce more units when the learning in production is slow, $b=0.25$, it is recommended to restore the process more frequently. Jaber and Guiffrida (2004) considered one lot and $n=1$, while Jaber and Guiffrida (2008) considered one lot and $n>1$. The effect of restoration is somewhat insignificant when learning in production is fast, $b=0.75$. The rationale for this is that as learning becomes faster, $b$ increases from 0.25 to 0.75 , the process benefits from producing in smaller lots, which also results in fewer items to rework. For a given learning index, say $b=0.25$, the process benefits more from producing in more lots. To illustrate, assume that a firm needs to produce a lot of 200 units, then should it produce the 200 units in 1 lot, 2 lots, etc.? By looking into Table 5.2, the firm will produce in 2 lots of 106 each since $2 \times 106=212$ is closer to 200 than 144 . Furthermore, the performance on the learning curve would be better. To illustrate, refer to Table 5.3, where the corresponding performance for unit number 144 in Table 5.2 on the learning curve is 3.606, whereas the performance time of unit 106 when 2 lots are produced is 2.654, which is better (lower). 
Table 5.2. Optimum values of the lot size, $x^{*}$, with learning in production, no learning in rework, and no forgetting.

\begin{tabular}{|l|l|l|l|l|l|l|l|l|l|}
\hline \multicolumn{1}{|c|}{$x^{*}$} & \multicolumn{3}{c|}{$\boldsymbol{b}=\mathbf{0 . 2 5}$} & \multicolumn{3}{c|}{$\boldsymbol{b}=\mathbf{0 . 5}$} & \multicolumn{3}{c|}{$\boldsymbol{b = 0 . 7 5}$} \\
\hline$\varepsilon=0.0$ & $n=1$ & $n=2$ & $n=3$ & $n=1$ & $n=2$ & $n=3$ & $n=1$ & $n=2$ & $n=3$ \\
\hline No. of Lots & & & & & & & & & \\
\hline 1 & 144 & 251 & 347 & 100 & 159 & 208 & 65 & 97 & 122 \\
\hline 2 & 106 & 185 & 256 & 56 & 88 & 116 & 25 & 37 & 47 \\
\hline 3 & 96 & 166 & 230 & 47 & 74 & 97 & 20 & 30 & 38 \\
\hline 4 & 89 & 155 & 215 & 42 & 66 & 86 & 17 & 26 & 32 \\
\hline 5 & 85 & 148 & 204 & 38 & 61 & 79 & 16 & 23 & 29 \\
\hline
\end{tabular}

Table 5.3. Performance on the learning curve for the last unit produced as per Table 5.2.

\begin{tabular}{|l|l|l|l|l|l|l|l|l|l|}
\hline \multicolumn{1}{|c|}{$t^{*}$} & \multicolumn{3}{|c|}{$b=\mathbf{0 . 2 5}$} & \multicolumn{3}{c|}{$\boldsymbol{b = 0 . 5}$} & \multicolumn{3}{c|}{$\boldsymbol{b = \mathbf { 0 . 7 5 }}$} \\
\hline$\varepsilon=0.0$ & $n=1$ & $n=2$ & $n=3$ & $n=1$ & $n=2$ & $n=3$ & $n=1$ & $n=2$ & $n=3$ \\
\hline No. of Lots & & & & & & & & & \\
\hline 1 & 3.606 & 3.139 & 2.895 & 1.5 & 1.191 & 1.04 & 0.761 & 0.566 & 0.475 \\
\hline 2 & 2.654 & 2.311 & 2.131 & 0.833 & 0.661 & 0.577 & 0.294 & 0.218 & 0.183 \\
\hline 3 & 2.389 & 2.08 & 1.918 & 0.698 & 0.554 & 0.484 & 0.234 & 0.174 & 0.146 \\
\hline 4 & 2.232 & 1.943 & 1.792 & 0.623 & 0.495 & 0.432 & 0.202 & 0.150 & 0.126 \\
\hline 5 & 2.122 & 1.847 & 1.703 & 0.573 & 0.455 & 0.397 & 0.181 & 0.135 & 0.113 \\
\hline
\end{tabular}

The average time to process a unit, computed from (5.5) as $\sum_{i=1}^{N} T\left(x_{i}, n\right) / \sum_{i=1}^{N} x_{i}$, when the 200 units (where $b=0.25, \varepsilon=0.0$, and $n=1$ ) are processed in a single lot is 3.678 , in 2 lots of 100 units each is 3.615 , in 3 lots of 66.667 each is 3.592 , in 4 lots of 50 each is 3.581 , and in 5 lots of 40 each is 3.574 . The reduction in the average processing time benefits from the transfer of learning between subsequent lots and from having less defective items to rework as the lots are of smaller sizes. If learning becomes faster, i.e. $b=0.75$, then the average unit processing times would be $1.138,0.954,0.889,0.856$, and 0.835 , respectively. Repeating the same example (200 units, $b=0.25$, and $\varepsilon=0.0$ ) for $n=3$ ( i.e. three process restoration instances per lot) the average processing times improve by reducing the time from $3.678,3.615,3.592,3.581$, and 3.574 to $3.592,3.569,3.562,3.558$, and 3.555 , respectively, corresponding to improvement in average performance of $2.34 \%, 1.27 \%, 0.84 \%, 0.64 \%$ and $0.53 \%$. The behavior was similar for the case 
when $b=0.75, \varepsilon=0.0$, and $n=3$, the average unit processing times would reduce to 0.889 , $0.822,0.799,0.787$, and 0.780 , respectively. The percentage improvement in performance is greater when process restoration is applied in the presence of fast learning than for slow learning; where the average improvement in performance is $21.88 \%, 13.84 \%, 10.12 \%, 8.06 \%$, and $6.59 \%$.

\subsubsection{Learning in the rework process $(\varepsilon=0.25)$}

In this section, the second case of no forgetting is considered, which is learning in production and rework processes. Since $u_{i}=(i-1) x$ and $v_{i}=(i-1) \frac{\rho x^{2}}{2 n}$, where $i=1,2, \ldots, N$ in (5.17), the optimum lot size is computed as:

$$
x^{*}=\left(\frac{T_{1} n b\left(i^{1-b}-(i-1)^{1-b}\right)}{r_{1} \rho\left(i^{1-\varepsilon}-(i-1)^{1-\varepsilon}\right)(1-2 \varepsilon)\left(\frac{\rho}{2 n}\right)^{-\varepsilon}}\right)^{\frac{1}{1+b-2 \varepsilon}}
$$

Equation (5.23) corresponds to the lot size that minimizes the total time to produce and rework a lot. Any deviation from the optimum value will deteriorate performance. As shown in Table 5.4, learning in the rework process, provides the opportunity of producing more units in a lot. By comparing the values in Table 5.4 to those in Table 5.2, one can observe that with learning in production and rework processes, the number of units that could be produced in a lot and still move down on the learning curve, increases. For example, when the number of lots is 1 with no process restoration $(n=1)$, the process can improve on the learning curve up to unit 144 in Table 5.2, beyond which if production continues, performance deteriorates, because of the convex 
behavior of the learning curve. This is typical in the construction industry when towards the end of a project, most of the workforce are correcting mistakes rather pouring concrete. Whereas when there is learning in rework $(0<\varepsilon<0.5)$ the learning curve continues for 5.5 times more (from 144 to 794) in Table 5.3. Similarly, for $n=2$ and 3, the learning curve continues for 6.3 (from 251 to 1587 ) and 6.8 (from 347 to 2381) times more, respectively. The performance on the learning curve corresponding to the values of $x^{*}$ in Table 5.4 are given in Table 5.5. Table 5.4 shows that the optimum lot size is not different when $\varepsilon=b=0.25$ regardless of the number of lots, i.e. $1,2, \ldots$, and 5 . This is because the terms $\left(i^{1-b}-(i-1)^{1-b}\right)$ and $\left(i^{1-\varepsilon}-(i-1)^{1-\varepsilon}\right)$ eliminates one another in (5.23) when $\varepsilon=b$.

Table 5.4. Optimum values of the lot size, $x^{*}$, with learning in production and rework, and no forgetting.

\begin{tabular}{|c|c|c|c|c|c|c|c|c|c|}
\hline$x^{*}$ & \multicolumn{3}{|c|}{$b=0.25$} & \multicolumn{3}{|c|}{$b=0.5$} & \multicolumn{3}{|c|}{$b=0.75$} \\
\hline$\varepsilon=0.25$ & $n=1$ & $n=2$ & $n=3$ & $n=1$ & $n=2$ & $n=3$ & $n=1$ & $n=2$ & $n=3$ \\
\hline \multicolumn{10}{|l|}{ No. of Lots } \\
\hline 1 & 794 & 1587 & 2381 & 299 & 503 & 682 & 132 & 200 & 256 \\
\hline 2 & 794 & 1587 & 2381 & 182 & 306 & 414 & 47 & 72 & 92 \\
\hline 3 & 794 & 1587 & 2381 & 159 & 267 & 363 & 38 & 58 & 74 \\
\hline 4 & 794 & 1587 & 2381 & 146 & 246 & 333 & 33 & 51 & 65 \\
\hline 5 & 794 & 1587 & 2381 & 137 & 230 & 312 & 30 & 46 & 58 \\
\hline
\end{tabular}

Table 5.5. Performance on the learning curve for the last unit produced as per Table 5.4.

\begin{tabular}{|l|l|l|l|l|l|l|l|l|l|}
\hline$t^{*}$ & \multicolumn{3}{l}{$\boldsymbol{b = \mathbf { 0 . 2 5 }}$} & \multicolumn{3}{l|}{$\boldsymbol{b}=\mathbf{0 . 5}$} & \multicolumn{3}{l|}{$\boldsymbol{0 . 7 5}$} \\
\hline$\varepsilon=0.25$ & $n=1$ & $n=2$ & $n=3$ & $n=1$ & $n=2$ & $n=3$ & $n=1$ & $n=2$ & $n=3$ \\
\hline No. of Lots & & & & & & & & & \\
\hline 1 & 2.826 & 2.376 & 2.147 & 1.156 & 0.891 & 0.766 & 0.641 & 0.469 & 0.391 \\
\hline 2 & 1.926 & 1.62 & 1.464 & 0.614 & 0.473 & 0.407 & 0.262 & 0.192 & 0.159 \\
\hline 3 & 1.689 & 1.42 & 1.283 & 0.504 & 0.388 & 0.334 & 0.206 & 0.151 & 0.126 \\
\hline 4 & 1.551 & 1.304 & 1.178 & 0.443 & 0.342 & 0.294 & 0.177 & 0.129 & 0.108 \\
\hline 5 & 1.465 & 1.224 & 1.106 & 0.403 & 0.311 & 0.267 & 0.158 & 0.115 & 0.096 \\
\hline
\end{tabular}

Repeating the same numerical example of producing 200 units when $b=0.25$ and $n=1$, but for $\varepsilon=0.25$, the average times to process a unit are 3.510 when producing in 1 lot, 3.555 in 2 lots, 
3.565 in 3 lots, 3.568 in 4 lots, and 3.568 in 5 lots registering improvements of $4.57 \%, 1.65 \%$, $0.76 \%, 0.37 \%$, and $0.16 \%$. This suggests, when the rework learning index is $\varepsilon=0.25$, improvement in the average performance is more recognizable when the 200 units are produced in less lots than in more lots.

Note that the benefit from learning in production and rework is more apparent when more units are produced per lot. For example, it is assumed that instead of producing 200 items the firm has a commitment of producing 2000 units, when $b=0.25$ and $n=1$, with $\varepsilon=0.25$ instead of 0.0 , improvements in the unit average processing time of $57.24 \%, 42.44 \%, 33.58 \%, 27.67 \%$ and $23.44 \%$ were registered. For the same input parameters except for $n=3$, improvements in the unit average processing times of $33.58 \%, 20.27 \%, 14.26 \%, 10.87 \%$ and $8.70 \%$ were registered. Repeating the same numerical example for 2000 units, $b=0.75, \varepsilon=0.25$ and $n=3$, improvements in the unit average processing times by $68.27 \%, 59.27 \%, 52.75 \%, 47.60 \%$ and $43.35 \%$ were registered. This suggests that when learning in rework is slow $(\varepsilon=0.25)$, maximum benefit can be attained when learning in production is fast $(b=0.75)$ and process restoration is more frequent. The best performance, average time to process a unit $(0.248)$, was recorded when the 2000 units were processed in 5 equal lots, where $b=0.75, \varepsilon=0.25$, and $n=3$. Whereas the worst (3.497) was when they were processed in a single lot, where $b=0.25$, $\varepsilon=0$, and $n=1$. 


\subsubsection{Learning in the rework process $(\varepsilon=0.5)$}

The learning curve in (5.11) reaches a plateau value, i.e. $t\left(x_{i}\right) \rightarrow r_{1}\left(i^{1 / 2}-(i-1)^{1 / 2}\right)\left(\frac{2 \rho}{n}\right)^{1 / 2}$ as $x_{i} \rightarrow \infty$ for $\varepsilon=0.5$ according to lemma 3 where $i$ is the lot number. Considering $r_{1}=5$ units of time and $\rho=0.001$, the process plateaus at the values summarized in Table 5.6.

Table 5.6. Plateau values of the composite learning curve when there is no forgetting in the learning and rework processes, for all $b>0$, and $\varepsilon=0.5$.

\begin{tabular}{|l|l|l|l|}
\hline $\boldsymbol{t}(\boldsymbol{x})$ & $\boldsymbol{n}=\mathbf{1}$ & $\boldsymbol{n = 2}$ & $\boldsymbol{n}=\mathbf{3}$ \\
\hline Lot \#1 & 0.224 & 0.158 & 0.129 \\
\hline Lot \#2 & 0.093 & 0.065 & 0.053 \\
\hline Lot \#3 & 0.071 & 0.500 & 0.041 \\
\hline Lot \#4 & 0.060 & 0.042 & 0.035 \\
\hline Lot \#5 & 0.053 & 0.037 & 0.030 \\
\hline
\end{tabular}

The results in Table 5.6 suggest that, for a given lot number, when restoration is more frequent $(n>1)$, plateauing is broken where the process moves to a lower plateau value each time the process is restored therefore the plateauing behavior that is usually observed in the learning data can be further overcome when the process benefits from the transfer of learning between lots, that is, as the number of lots increases.

\subsubsection{Learning in the rework process $(0.5<\varepsilon<1)$}

If the learning index in the rework process is $0.5<\varepsilon<1$, the process improves continuously, as more lots are produced. A well-known example of this situation is the Wright's learning curve (1936). The behavior of the aggregated learning curve has been previously explained, for the first lot only, in Jaber and Guiffrida (2004). As more lots are produced, the same behavior of the 
learning curve is observed as that for the first lot, except performance moves further down on the learning curve as a result of the transfer of learning between lots.

\subsubsection{Forgetting Cases}

In this section, an intermittent production/rework process in examined during which forgetting occurs as a result of breaks. That is, production/rework processes are intermittent. This allows for some of the experience gained from previous lots to be lost because of the time breaks, which are of two types:

i) a cycle (i.e. production + rework) is interrupted for a period of time to release the workstation to perform another dissimilar job, or for resting, or both.

ii) a worker is shifted to reworking the defective items generated in the production segment of a cycle, where it is assumed that a worker is flexible to perform both production and rework tasks. It is also assumed that production and rework are dissimilar tasks and the period for reworking defective items is considered as a break over which some of the experience gained from production segments of the previous or prior cycles is lost.

In the numerical examples performed in this section, it is assumed that the length of the interruption period that separates two subsequent cycles is fixed at 200 units of time relative to $B=2000$ units of time, which is the time for total forgetting to occur. The interruption time between two production periods, $i$ and $i+1$, would be the time to rework the defective items produced in the current cycle, $R t_{i}$, plus the 200 units. Similarly, the interruption time between 
two rework periods, $i$ and $i+1$, would be the time to produce the items in the next cycle, $P t_{i+1}$, plus the 200 units. Then, we have the breaks computed as follows:

$\varphi_{r, i+1}=200+P t_{i+1}$

$\varphi_{p, i+1}=200+R t_{i}$

This process is illustrated in Figure 5.1 with the current production and rework lots. The experience from production $(P L(i))$ and rework $(R L(i))$ is transferred to cycle $i$ from the previous cycle and is measured in units, represented by $u(i)$ and $v(i)$, respectively, and $x$ and $d(x)$ depict the lot size and number of defective items, respectively.

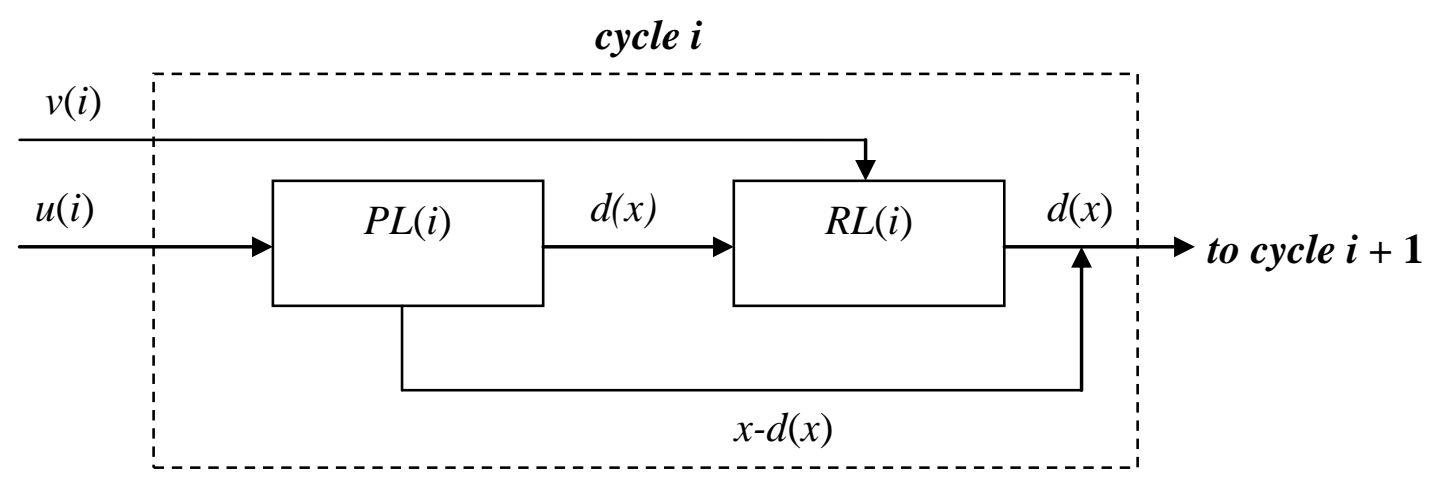

Figure 5.1. Cycle $i$ consists of production and rework processes. The experiences $u(i)$ and $v(i)$ are transferred to the current cycle from previous runs.

The next section investigates the effect of forgetting in the production and rework processes for different learning rates in production and rework, different number of process restorations, and for consecutive lots. 


\subsubsection{No learning in the rework process $(\varepsilon=0.0)$}

To illustrate the effect of forgetting on the QLC, when there is no learning in the rework process, with the assumption that 2000 items are produced in 5 lots, the following steps are taken:

1. Calculate the time to process the 400 units in the first lot by integrating equation (4.11) over the proper limits.

2. Calculate the time to rework the defective items generated from producing the first lot, $r \rho(400)^{2} / 2 n$, where $r_{1}=r$. The amount of the experience transferred from a cycle to the next, is calculated from (4.4) to (4.6) by (5.24) and (5.25) for the production and rework breaks, respectively.

3. Calculate the lot size that minimizes (5.6) and the corresponding time on the learning curve.

4. Repeat the above steps for the remaining lots.

Note that in this example the amount of experience transferred from rework is not calculated since we assume no learning in rework, i.e. $\varepsilon=0.0$. In later sections, where $\varepsilon>0.0$, step 2 is also performed for rework. The results are summarized in Table 5.7 and Table 5.8. Comparing these results with their corresponding ones in Table 5.2 and Table 5.3, where there was full transfer of learning between subsequent lots (i.e. no forgetting), it can be noticed that larger lot size values are produced and the performance deteriorates as a result. 
Table 5.7. Optimum values of the lot size, $x^{*}$, with learning and forgetting in production and no learning in rework.

\begin{tabular}{|l|l|l|l|l|l|l|l|l|l|}
\hline$x^{*}$ & \multicolumn{4}{|l|}{$\boldsymbol{b}=\mathbf{0 . 2 5}$} & \multicolumn{3}{l|}{$\boldsymbol{b}=\mathbf{0 . 5}$} & \multicolumn{3}{l|}{$\boldsymbol{b}=\mathbf{0 . 7 5}$} \\
\hline$\varepsilon=0.0$ & $n=1$ & $n=2$ & $n=3$ & $n=1$ & $n=2$ & $n=3$ & $n=1$ & $n=2$ & $n=3$ \\
\hline Lot \#1 & 144 & 251 & 347 & 100 & 159 & 208 & 65 & 97 & 122 \\
\hline Lot \#2 & 111 & 182 & 258 & 81 & 119 & 155 & 54 & 73 & 90 \\
\hline Lot \#3 & 108 & 170 & 238 & 81 & 116 & 149 & 53 & 72 & 88 \\
\hline Lot \#4 & 108 & 168 & 233 & 81 & 115 & 148 & 53 & 72 & 88 \\
\hline Lot \#5 & 108 & 168 & 232 & 81 & 115 & 148 & 53 & 72 & 88 \\
\hline
\end{tabular}

Table 5.8. Performance on the learning curve for the last unit produced as per Table 5.7.

\begin{tabular}{|l|l|l|l|l|l|l|l|l|l|}
\hline$t^{*}$ & \multicolumn{1}{|l}{$\boldsymbol{b = 0 . 2 5}$} & \multicolumn{1}{l}{$\boldsymbol{b}=\mathbf{0 . 5}$} & \multicolumn{2}{l|}{$\boldsymbol{b}=\mathbf{0 . 7 5}$} & \\
\hline$\varepsilon=0.0$ & $n=1$ & $n=2$ & $n=3$ & $n=1$ & $n=2$ & $n=3$ & $n=1$ & $n=2$ & $n=3$ \\
& & & & & & & & & \\
\hline Lot \#1 & 3.606 & 3.139 & 2.895 & 1.5 & 1.191 & 1.04 & 0.761 & 0.566 & 0.475 \\
\hline Lot \#2 & 3.439 & 2.967 & 2.746 & 1.406 & 1.092 & 0.952 & 0.703 & 0.506 & 0.422 \\
\hline Lot \#3 & 3.426 & 2.937 & 2.712 & 1.403 & 1.083 & 0.941 & 0.702 & 0.503 & 0.419 \\
\hline Lot \#4 & 3.425 & 2.932 & 2.704 & 1.403 & 1.082 & 0.939 & 0.702 & 0.503 & 0.418 \\
\hline Lot \#5 & 3.425 & 2.931 & 2.702 & 1.403 & 1.082 & 0.939 & 0.702 & 0.503 & 0.418 \\
\hline
\end{tabular}

By comparing the first five lots of Table 5.7 to Table 5.2, it can be seen that the forgetting effects are observed in lots 2-5. When there is forgetting, it takes more units to reach the optimum performance on the learning curve, where performance is slower than when there is no forgetting. To better illustrate the effect of forgetting; let us consider the case when the 2000 units are produced in 5 equal lots of 400 units each. For the case when $b=0.25, \varepsilon=0.0$, and $n=1$ and no forgetting, the average time per unit to produce and rework a unit is $3.318,2.528$, $2.320,2.18$, and 2.115 for lots $1,2,3,4$ and 5, respectively. When there is forgetting, the average time per unit to produce and rework a unit is $3.318,3.086,3.075,3.074$, and 3.074 , for lots 1,2 , 3, 4 and 5, respectively, corresponding to a deterioration in the average performance of $0 \%$, $22.08 \%, 32.54 \%, 41.01 \%$, and $45.34 \%$. Repeating the same numerical example but for $n=3$, the average time per unit deteriorates by $0 \%$ (from 3.108 to 3.108 ), $19.7 \%$ (from 2.219 to 2.656 ), $31.3 \%$ (from 1.984 to 2.605 ), $40.4 \%$ (from 1.848 to 2.594 ), and $47.8 \%$ (from 1.754 to 2.592 ). 
Now, repeating the above example for $b=0.75$, when $\varepsilon=0.0$ and $n=1$, the average time per unit deteriorates by $0 \%$ (from 1.206 to 1.206 ), $16.6 \%$ (from 0.904 to1.0544), 19.6\% (from 0.881 to 1.054 ), $21.1 \%$ (from 0.871 to 1.054 ), and $21.9 \%$ (from 0.864 to 1.054 ) when there is forgetting. For $n=3$, the average time per unit deteriorates by $0 \%$ (from 0.732 to 0.732 ), $31.7 \%$ (from 0.392 to 0.517 ), $40.4 \%$ (from 0.366 to 0.514 ), $45.1 \%$ (from 0.354 to 0.514 ), and $48.1 \%$ (from 0.347 to 0.514 ). These results suggest that forgetting can significantly impede the performance.

\subsubsection{Learning in the rework process $(\varepsilon=0.25)$}

Here, we investigate the effects of learning in production and rework when forgetting exists. Let us consider the numerical example where 2000 units are produced in 5 equal lots of 400 units each, where $b=0.25, \varepsilon=0.25$, and $n=1$, production break is 200 , and $B=2000$. By comparing the average time to process a unit of this case to the one with the same parameters except for $\varepsilon=0.0$, the average time per unit improves by $13.92 \%$ (from 3.318 to 2.856 ), $19.81 \%$ (from 3.086 to 2.475 ), $20.91 \%$ (from 3.075 to 2.432 ), $21.18 \%$ (from 3.074 to 2.423 ), and $21.24 \%$ (from 3.074 to 2.421 ), when $\varepsilon$ increases from 0.0 to 0.25 . Repeating the same example for $n=3$ the average time per unit improves by $4.18 \%$ (from 3.108 to 2.978 ), $7.42 \%$ (from 2.656 to 2.459), $8.52 \%$ (from 2.605 to 2.383 ), $8.91 \%$ (from 2.594 to 2.363 ), and $9.10 \%$ (from 2.592 to 2.356), when $\varepsilon$ increases from 0.0 to 0.25 . These results suggest that with learning in rework and more frequent restorations, the effect of forgetting can be reduced. Further improvement can be claimed when $b$ increases from 0.25 to 0.75 , where the average time per unit per lot improves by $79.75 \%$ (from 2.978 to 0.603 ), $85.81 \%$ (from 2.459 to 0.349 ), $85.9 \%$ (from 2.383 to 0.336 ), $85.86 \%$ (from 2.363 to 0.334 ), and $85.82 \%$ (from 2.356 and 0.334 ), when $n=3, \varepsilon=0.25,400$ 
units produced per lot, 200 is the break length and $B=2000$. These results suggest that when learning in rework is present, the process improves more than when there is only learning in production. Further improvements can be claimed when the process is restored more frequently and when learning in production is fast. This reduces the cycle length and, therefore, reduces the impact of forgetting (see Jaber \& Bonney, 2011).

\subsubsection{Learning in the rework process $(\varepsilon=0.5)$}

The learning curve in (5.11) reaches the plateau value of $t\left(x_{i}\right) \rightarrow r \sqrt{2 \frac{\rho}{n}}$ as $x_{i} \rightarrow \infty$ for $\varepsilon=0.5$ according to lemma 3 where $n$ is the number of restorations and the process undergoes forgetting. Considering $r_{1}=5$ units of time and $\rho=0.001$, the process plateaus in each of the 5 lots at $0.224(n=1), 0.156(n=2)$, and $0.129(n=3)$. This indicates that more restorations in the process push the plateau value of a learning curve to lower values. This may entice managers to follow such a policy to unlock the improvement of their processes.

Forgetting causes the plateau level to increase. We compare $t\left(x_{i}\right) \rightarrow r_{1} \sqrt{2 \frac{\rho}{n}}$ (see Lemma 4) and $t\left(x_{i}\right) \rightarrow r_{1}\left(i^{1 / 2}-(i-1)^{1 / 2}\right)\left(\frac{2 \rho}{n}\right)^{1 / 2}$ (with no forgetting) when $x_{i} \rightarrow \infty$ in section 5.3.4. These two formulas correspond to the cases of forgetting and no forgetting, respectively. The ratio of the second to the first gives: 
$\frac{r_{1}\left(i^{1 / 2}-(i-1)^{1 / 2}\right)\left(\frac{2 \rho}{n}\right)^{1 / 2}}{r_{1} \sqrt{2 \frac{\rho}{n}}}=i^{1 / 2}-(i-1)^{1 / 2}<1 \forall i \neq 1$, which is always less than one for any lot except the first one. Therefore, the process will plateau at a higher time value under the effect of forgetting, which is an indication of the loss of profit due to forgetting.

\subsubsection{Learning in the rework process $(0.5<\varepsilon<1)$}

Let us consider the numerical example where 2000 units are produced in 5 equal lots of 400 units each, where $b=0.25, \varepsilon=0.75$, and $n=1$, production break is 200 , and $B=2000$. The average time per unit is $2.609,2.095,2.002,1.975$, and 1.965 for lots 1 to 5 , respectively. The values improve over the case when all the parameters are the same except for $\varepsilon=0.25$ by $8.65 \%$, $15.35 \%, 17.68 \%, 18.49 \%$, and $18.85 \%$, respectively. The average time to process a unit improves significantly when $b=\varepsilon=0.75$ and $n=3$. The percentages of the improvement in performance are $79.85 \%, 89.21 \%, 89.56 \%, 89.52 \%$, and $89.01 \%$, respectively.

\subsection{Results}

The results of this Phase shed light on an intermittent production process which is restored for the quality maintenances and it entails the forgetting effects. The effect of restorations on the performance is represented in Figure 5.2 for a composite process of production, with $b=0.25$, and rework, with $\varepsilon=0.0$. More restorations decrease the number of defective items and move the optimum lot size forward on the horizontal axis. The optimum lot size increases from 144 to 251 , 347, 437, and 523 for $n=1,2,3,4$ and 5, respectively, which allows producing in larger lots. 
The process also can benefit from producing in smaller lots more frequently. The more the process is restored; the lower is the average unit time (better performance).

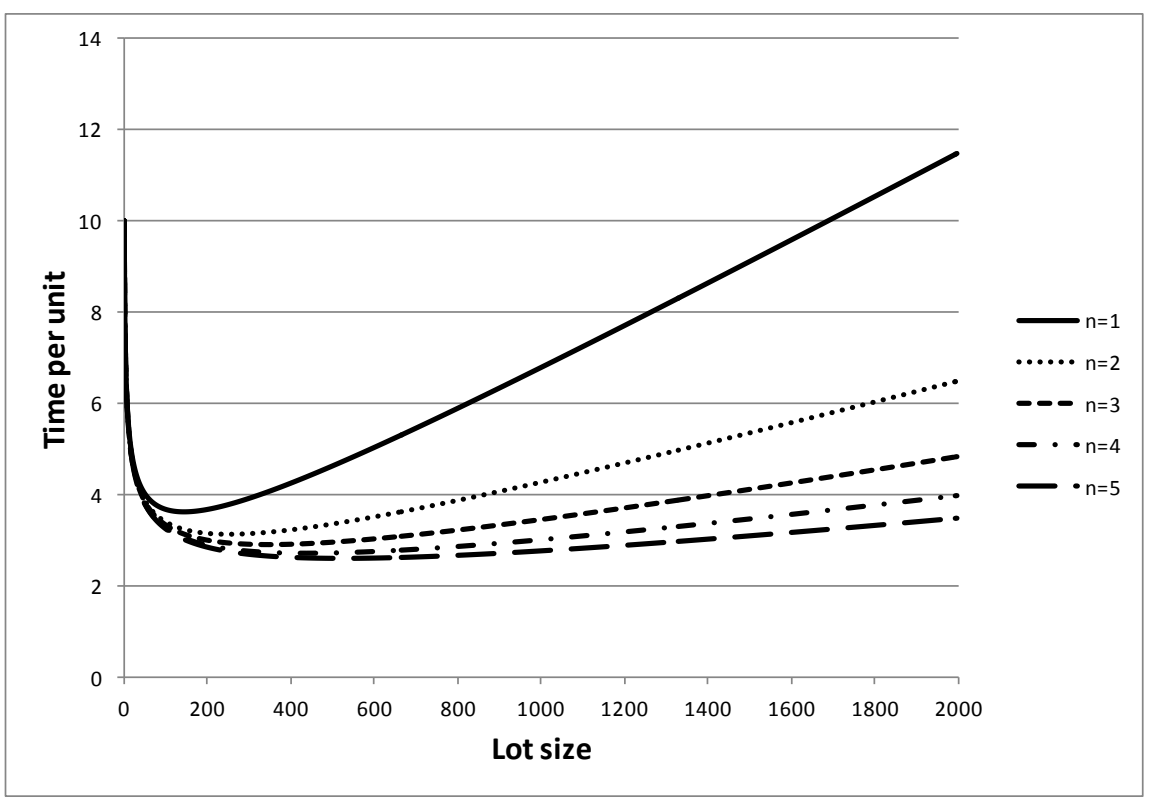

Figure 5.2. The effect of restorations on the average unit time of the process when $b=0.25$ and $\varepsilon=0.0$. A single lot for $(i=1)$ is restored for $n$ times, $n=1, \ldots, 5$.

Figure 5.3 illustrates the effect of forgetting on the optimum lot size with different number of restorations where $b=0.25$ and $\varepsilon=0.0$. Without forgetting, the optimum lot size continues to decrease due to learning effects. With forgetting, the optimum lot size reaches a plateau and ceases to reduce. The results indicate that more restorations increase the lot size due to producing less defective items. Figure 5.4 illustrates the effect of forgetting on the optimum time per unit in a cycle (i.e. production + rework) where $b=0.25$ and $\varepsilon=0.0$. Without forgetting, time per unit continues to decrease per lot. With forgetting, the improvement ceases due to the plateauing effect. More process restorations shift the curves down, which is an indication of a breakage in the plateau barrier and subsequently a reduction in the standard time of the process. 


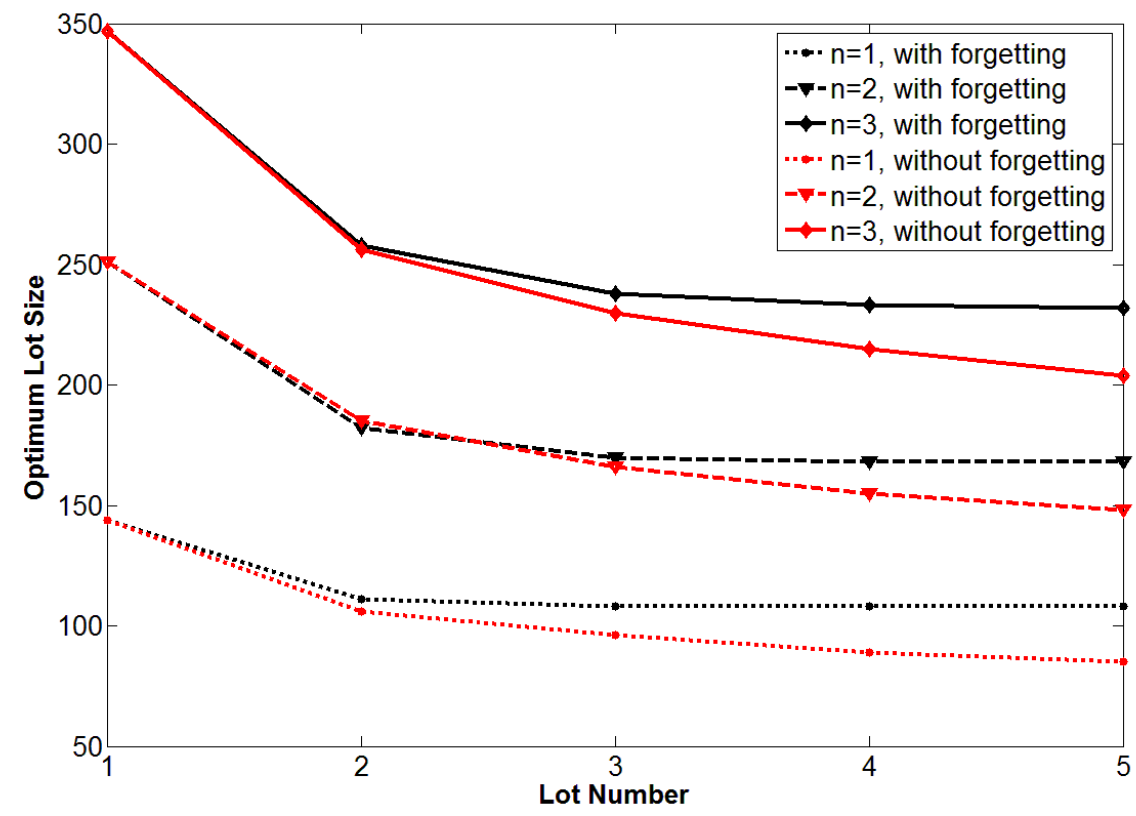

Figure 5.3. The effect of splitting a larger lot into smaller sublots with and without forgetting. The optimum lot size decreases with more lots. The forgetting effect prevents the lot size to decrease as per Table 5.2 and Table 5.7.

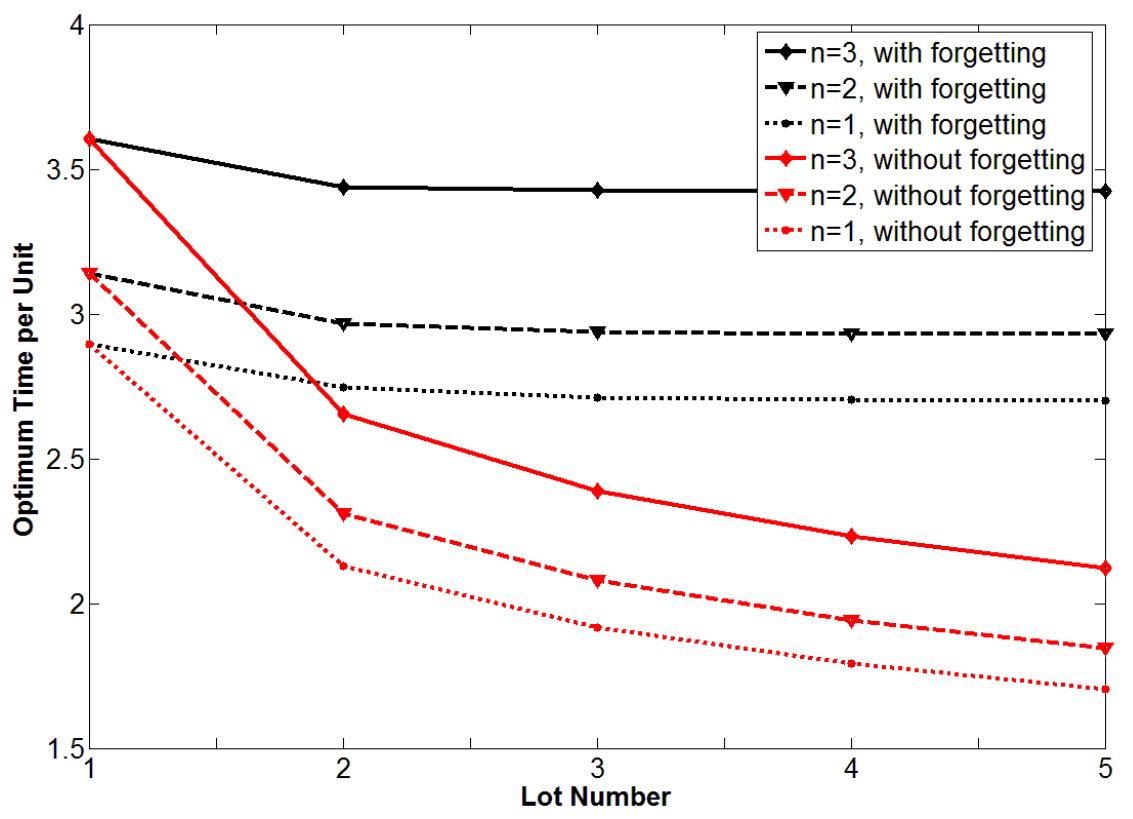

Figure 5.4. The effect of splitting a larger lot into sublots with and without forgetting. The plateau effects prevents the time per unit to decrease due to forgetting as per Table 5.3 and Table 5.8. 


\subsection{Discussion and conclusion}

Phase 2 of this research extended the quality learning curve (QLC) developed by Jaber and Guiffrida (2004, 2008) to account for the transfer of learning and forgetting effects in a production line with the quality effects. The QLC model of Jaber and Guiffrida (2004) considered a single production cycle that is not restored once it goes out of control. In a following study, Jaber and Guiffrida (2008) revised the QLC by allowing the process to be interrupted and restored as a policy to reduce the cumulative number of defective units in a cycle and to break the learning plateau barrier. This Phase extended these earlier works by considering several cycles performed sequentially and that forgetting may occur when these production cycles are separated by production breaks.

Similar to the previous models, the QLFC model demonstrated three different behaviors: convex, plateau, and continually improving. The results showed that when the rework learning index is half, i.e. $\varepsilon=0.5$, the process plateaus at a time smaller in the case of no forgetting and larger when forgetting occurs. This suggests that the process will operate at higher costs because of forgetting. When the rework learning index is larger than 0.5 , i.e. $\varepsilon>0.5$, the process continues to improve. The results also indicated that the performance improves with faster learning and frequent process restorations.

This Phase of the thesis would be helpful to set strategies for quality improvements, waste reduction, and for breaking the learning plateau barrier. The results of this Phase may also be used in scheduling workers in DRC systems where there is a tradeoff between flexibility and productivity. 
A significant amount of money is spent by industries, e.g., automobile manufacturers, for training (learning) and retraining (following forgetting) purposes (Jaber et al., 2003; Omar et al., 2011; Bapna et al., 2013; O'Leonard, 2013) to encounter the effects of forgetting. Using robotics and flexible manufacturing systems, worker flexibility, cross-training, sharing the experience, and assignment scheduling were also suggested to reduce the forgetting effects (Jaber \& Bonney, 1999; Heimerl \& Kolisch, 2010; Kang \& Hur, 2013). In a competitive and dynamic market, the capability of manufacturing companies to increase the learning abilities, and to reduce or anticipate the forgetting effects is vital for them in order to survive. This Phase of the thesis may help managers in identifying operations and processes that are subject to learning and forgetting effects and to properly estimate their associated costs. The QLFC model developed in this Phase could also be investigated in a more complex context such as production planning and control. 


\section{Phase 3: Investigating human error with learning-forgetting and fatigue- recovery}

This Phase of the thesis studies the interaction of human behavioral functions such as learningforgetting and fatigue-recovery that affect human reliability when performing a manual task. A mathematical model is developed that calculates the human error rate while performing an assembly job and accounts for the dynamic behavior of human reliability in a production process. The results of this Phase will be used in production planning in a DRC system in Phase 4.

\subsection{Introduction}

The costs of reworking or scrapping defective items are of concern to many manufacturers. Unnecessary rework and disposal costs of scrapped items require committing additional resources that increase production costs (Jenab et al., 2009). Reducing the worker's error rate reduces the number of defective items generated. According to a report by U.S. National Safety Council, in 1999, human error was a contributor to $80 \%$ of all industrial accidents, which caused \$98.5 billion of costs (Griffith \& Mahadevan, 2011). To counter the detrimental effects of human error, one has to study why and how an error occurs. Noroozi et al. (2013) identified several causes of human error in manufacturing environments, such as: lack of training, poor design, poor lighting or noise condition, improper tools or work layout, and unclear operating procedure. This indicates that human error is a multidimensional problem, which affects the reliability of the system and the quality of its output.

In order to measure and manage the reliability of labour intensive systems, it is necessary to assess the reliability of the workforce of such systems. According to Cacciabue (1998), human 
reliability analysis (HRA) predicts human error rates and evaluates the degradation to the human-machine system that is caused by declines in human functioning, operational procedures, and practices, and other system and human characteristics which affect the system behaviour .

In HRA models, human error probability (HEP) is calculated based on the operator's activities and his/her performance shaping factors (PSF) that are either related to the working environment, or the physical and mental capabilities of workers, or both. Identifying PSFs is then essential in HRA process (Di Pasquale et al., 2013).

HRA models are classified into two main categories of first and second generations. The first generation models are quantitative models such as the Technique for Human Error Rate Prediction (THERP) by Swan and Guttman (1983) and Human Cognition Reliability (HCR) by Hannaman et al. (1984). These models treat a worker as a mechanical element that does not have any interaction with his/her surrounding environment. For such models, HEP is determined from task characteristics and PSFs by fuzzy success/failure modes, and the causes and reasons of human error are not considered (Di Pasquale et al., 2013).

The second generation of HRA models are qualitative models that use methods like Cognitive Reliability Error Analysis Method (CREAM) by Hollnagel (1996) and Standardized Plant Analysis Risk HRA method (SPAR-H) by Boring and Blackman (2007). These models pay more attention to the causes of human error, and the dependency (interaction) of PSFs of one factor on (with) other factors. The cognitive models in this category, describe the man-machine interactions by considering a human cognition system in deciding situations where the operator is in possession of "awareness" and "judgement" (Di Pasquale et al., 2013). Unlike the first generation, the models of the second generation have not been empirically validated and in the 
majority of the proposed models, implicit functions relate the PSFs to numeric probabilities (Mosleh \& Chang, 2004; Di Pasquale et al., 2013). Based on the study of HRA models, it is concluded that the available HRA models have the following shortcomings: Firstly, there is no available empirical data to validate them. Secondly, they do not consider human behaviour objectively. Thirdly, and lastly, the available models are limited to distinct areas (such as nuclear power plants or transportation systems) and their HEP estimation depends on the applied methodology. Moreover, HRA models require an expert's judgment to determine/estimate the PSFs values (Griffith \& Mahadevan, 2011). Another problem with HRA models is that these models were developed primarily to analyze the causes and occurrences of accidents in the work environments that are restrictive in nature and subject to stringent measures. Therefore, they are more concerned about the outcome of a decision making process rather than analysing the dynamics of the error generation process. They also cannot be used to predict when and how often an error occurs. Therefore, the estimation of error and management of workers' reliability remain a concern in labour-intensive systems.

Reducing the cost associated with errors is achieved by proper measurement and control of PSFs. It has been shown that the error rate of a worker decreases as he/she accumulates more experience and it increases by fatigue (Giuntini, 2000; Myszewski, 2010). The aim of Phase 3 of the thesis is to model the error rate of a worker taking into account the PSFs of learning and physical fatigue and their opposites, forgetting and recovery. The error rate of a worker, on one hand, decreases as he/she accumulates more experience due to repetition in accordance with his/her learning curve (Giuntini, 2000), but at the same time, he/she accumulates fatigue, which increases the error rate (Myszewski, 2010; Michalos et al., 2013). Rest breaks, alleviate worker fatigue (Krajcarski \& Wells, 2008) and decrease the probability of committing mistakes/errors 
on a job, but at the same time, they cause forgetting (Anzanello \& Fogliatto, 2011; Froehle \& White, 2013), which is an error-increasing PSF (ElMaraghy et al., 2008). The combined effect of learning and forgetting along with fatigue and recovery has not been investigated in the HRA and human error modelling literature. The aim of this Phase therefore, is to contribute to HRA literature by presenting a mathematical model for human error analysis by considering the interaction of learning - forgetting and fatigue - recovery phenomena.

The rest of Section 6 is organized as follows: Section 6.2 presents a literature review for human reliability models. The methodology of Phase 3 is demonstrated in Section 6.3. Results are presented in Section 6.4 and discussed in Section 6.5.

\subsection{Human Error}

Kirwan (1992, p. 300) has defined failure or error as: "The failure to perform an act within the limits (of time, accuracy, etc.) required for safe system performance, or else the performance of a non-required act which interferes with system performance". Dhillon (2009) categorized the common human errors in engineering processes as: maintenance error, operator error, design error, assembly error, inspection error, handling error, and contributory error. Error in assembly has been associated with worker's capabilities such as knowledge and competence; worker's fatigue; task parameters such as workload and repetitiveness; and the work environment (ElMaraghy et al., 2008; Xin \& Fan-Sen, 2010; Michalos et al., 2013). These errors can occur as a result of performing tasks non-sequentially, using the wrong parts, apply the wrong force or torque, misalignments, loose joints, and missing parts (Michalos et al., 2013). 
In this thesis, human error is defined as a mistake in performing a task that results in producing a quality deficit. Errors are corrected at a cost and are considered to be affected by the worker's personal capabilities and the work characteristics. The worker's capability in performing a repetitive task, or competence, is presented by his/her learning curve. Also, the work characteristics such as work environment (e.g. noise and humidity) and severity, are represented by the worker's fatigue (Ji et al., 2006). Other parameters, that directly or indirectly affect these two factors are summarised in Table 6.1.

Table 6.1. Example of categorization of parameters affecting human error* as per ElMaraghy et al. (2008).

\begin{tabular}{ll}
\hline Parameters contribute to Learning & Parameters contribute to Fatigue \\
\hline Task repetitiveness and complexity & Task repetitiveness and complexity \\
Skill level & Task environment \\
Training & Skill level \\
Interruptions & Stress \\
Forgetting & Ergonomic design of workplace \\
Multitasking & Line speed \\
Job rotation & Worker personality \\
Task reallocation & Management strategy \\
Task reconfiguration & Task time \\
Work teams & Work teams \\
\hline
\end{tabular}

\subsubsection{Human error and learning-forgetting}

It has been documented in the literature that a worker's error rate declines with experience (Chand, 1989; Koulamas, 1992; Teng \& Thompson, 1996; Franceschini \& Galetto, 2002; Myszewski, 2010; Grosse \& Glock, 2013). However, experience as a result of learning has not been explicitly considered in HRA models. These models provide numerical measures (probability of committing an error) associated with a description of a worker's knowledge level or experience. For example, the probability of committing an error by an operator who is totally unfamiliar with a task is considered as 0.55 as suggested by Human Error Assessment and 
Reduction Technique (HEART) model whereas for an operator who is totally familiar with the task and performs it repetitively is 0.0004 (Smith, 2011). Although the HEART model distinguishes among the factors that (negatively or positively) contribute to error-making, including learning, it does not provide a methodology to rationalize this distinction. In another model, Empirical Technique to Estimate Operator Error (TESEO) by Bello and Colombari (1980), the skill of an operator is classified as "Expert", "Average", and "poorly trained" with "error scores" of $0.5,1$ and 3, respectively. Although TESEO emphasizes on the relationship between the level of expertise and error-making, the relationship is numerically derived from informed guesses, rather than empirically driven data (Geisinger, 2003). The lack of supporting evidence indicates that there is not enough justification in support of these scores and the way the accumulation of experience contributes to these scores.

Some researchers modelled error generation as a function of time. For example, Myszewski (2010) and Giuntini (2000) have suggested that the human error rate decreases as the number of repetitions increases with the time, according to a learning curve, but have not accounted for its opposite phenomenon, forgetting. Forgetting may occur during breaks or production interruptions (Globerson et al., 1989; Jaber \& Bonney, 1996; Anzanello \& Fogliatto, 2011; Froehle \& White, 2013) and it was identified as a cause of human error, which affects the quality of products (Reason, 1990; Kirwan, 1992; ElMaraghy et al., 2008). Since learning and forgetting are considered to be mirror images of one another, while learning improves quality by decreasing the occurrence of errors, forgetting tends to increase errors and impede the quality. 


\subsubsection{Human error and fatigue-recovery}

Fatigue is known as a contributor to human error generation (Granger \& Jen-Gwo Chen, 1994; Luczak \& Mueller, 1994; ElMaraghy et al., 2008; Michalos et al., 2013). Errors caused by fatigue have been observed in aviation (Helmreich, 2000; Isaac et al., 2002; Dhillon, 2009), transportation (Dhillon, 2007; Gander et al., 2011), healthcare (Dean et al., 2006; Barker \& Nussbaum, 2011), electronic industry (Yeow \& Nath Sen, 2003) and car manufacturing (Fritzsche et al., 2014). A study by National Aeronautics and Space Administration (NASA) revealed that $70 \%$ of all aviation accidents were related to fatigue (Helmreich, 2000). In the manufacturing sector, $26 \%$ of deficit items were shown to be related to human fatigue (Dodé, 2011). Although fatigue is acknowledged as a multidimensional problem and there is an increasing interest among scholars in this subject, it has not been considered explicitly in HRA models (Griffith \& Mahadevan, 2011). For example, in the TESEO model, while calculating the human error probability, fatigue could be considered as an "Ergonomics" scale factor. In this context, "Excellent ergonomics" is factored by 0.7 , "Good ergonomics" by 1, "Average ergonomics" by a number between 3 and 7, and "Very poor ergonomic" by 10. TESEO associates each of this ergonomic description with a probability of error. Clearly, excellent ergonomics has very low error probability while the poorest has the highest error probability. Readers are referred to Griffith and Mahadevan (2011) for information about the inclusion of fatigue in current HRA models Where they describe the current status of HRA methods towards fatigue as "fatigue is neither considered as a PSF in HRA models nor do current HRA methods adequately include explicit quantitative measures for the effects of fatigue”(p.1444). 
The contribution of physical fatigue in producing human error has been investigated by some researchers. For example, Myszewski (2010) and Giuntini (2000) provided an error rate curve model which suggests that human error increases as fatigue increases over time. Michalos et al. (2013) considered a physical fatigue score that is calculated using the fatigue model of Ma et al. (2009), where fatigue accumulates over time as more work is performed. Michalos et al. (2013) then used the utility function of ElMaraghy et al. (2008) to calculate the corresponding error rate but none of the above models have considered how recovery from fatigue affects the error rate in assembly workers.

Recovery could be defined as the time needed by an individual to return to the pre-stress level of functioning following the termination of the stressor (Jansen et al., 2002). Work breaks interrupt the production process and decrease the available time of production, but they are necessary to alleviate some of the accumulated fatigue and decrease the boredom (Di Pasquale et al., 2013). In this context, recovery has the opposite effect of fatigue on worker's performance; i.e., it decreases the error rate and the risk of accidents (Tucker, 2003).

The above literature review reveals that there is an increasing interest in evaluating the learning and fatigue contribution in producing human error but there is no research available that incorporates learning and fatigue together with their reverse associated functions, forgetting, and recovery, in producing human error. The present Phase of the research aims to cover this gap by considering a repetitive assembly task in which a worker experiences learning and forgetting, and fatigue and recovery. The LFFRM developed in Phase 1 measures a worker's performance for the overlapping learning-forgetting and fatigue-recovery processes however, it did not account for the error rate of the worker and implicitly assumed that all items produced/assembled 
conform to quality. The current Phase overcomes this limitation and addresses how human error can generate quality deficit items in the assembly process. It utilizes LFFRM model to capture the interactions of learning-forgetting and fatigue-recovery phenomenon and then incorporates provisions for estimating the human error rate.

\subsection{Methodology}

Workers performing tasks on an assembly line are usually subjected to learning and forgetting and fatigue and recovery, simultaneously. Fatigue and recovery represent the general physical workloads associated with the tasks performed and the work environment. When a worker starts a task, he/she starts with a high initial error rate and a low initial cumulative experience. As time passes on the job, cumulative experience increases and error rate decreases as the worker moves down on his/her learning curve. However, with time, the worker accumulates general body fatigue that increases the error rate. To elevate fatigue, a learning (work) session is followed by a break. During this break, some of the knowledge acquired could be lost because of forgetting as a result of interruption.

In this Phase of the research, a model is developed to account for the contribution of learning and fatigue in generating human error along with the LFFRM model developed in 4. The normalized functions and the procedure provided by ElMaraghy et al. (2008) are utilised to develop this model.

To estimate the contribution of learning in decreasing the error probability, a normalized learning score is defined as: 
where $N L S$ is the normalized learning score, $t_{x}$ is the time to perform the $x^{\text {th }}$ repetition of a task (the real time), and $T_{1}$ is the time to perform the first repetition. Equation (6.1) suggests that $N L S$ (and its contribution to worker error) decreases as $t_{x}$ decreases. Equation (6.1) also indicates that the error rates of inexperienced operators are higher than those of experienced ones. From (6.1), the learning utility function is presented by:

$u_{l}=\alpha \times N L S$

where $\alpha$ is a constant multiplier and $0<u_{l} \leq 1$. In (6.2), it is assumed that learning induced error is at its maximum value, i.e. $u_{l}=1$ wherever there is no learning acquired. In this condition, $t_{x}$, in (6.1) is equal to the time required to assemble the very first unit, $t_{x}=T_{1}$ and $N L S=1$. Therefore, the maximum learning induced error rate from (6.2), $u_{l}=1$ is obtained if $\alpha=1$.

To estimate the contribution of fatigue to the error probability, a normalized fatigue score is used as:

$$
N F S=F(t) / F_{\max }
$$

where NFS is the normalized fatigue score, $F(t)$ is the accumulated fatigue at time $t$, which is calculated from (4.9), and $F_{\max }$ is the maximum fatigue, that for the purpose of this research is chosen as 1 (or 100\%). Equation (6.3) suggests that NFS (and its contribution to worker error) 
increases as fatigue $F$ increases over time, where $0<N F S<1$. The fatigue utility function is presented by:

$u_{f}=\beta \times N F S$

where $\beta$ is a constant multiplier and $0<u_{f} \leq 1$. It is assumed that the fatigue induced error rate is at its maximum value, i.e. $u_{f}=1$ whenever fatigue is $100 \%$ and therefore, $\beta=1$. The utility function that describes the human error rate with learning and fatigue effects is given as (ElMaraghy et al., 2008; Michalos et al., 2013):

$U(l, f)=u_{l} \times w_{l}+u_{f} \times w_{f}$

where $w_{l}$ and $w_{f}$ are the user defined weights, such that $w_{l}+w_{f}=1$, and $u_{l}$ and $u_{f}$ are the learning and fatigue utility functions as given in (6.2) and (6.4), respectively. The relative importance of PSFs (weights) in equation (6.5) can be determined by expert judgment, aspiration level of the workers, previous experience, or may be obtained from applying the Analytic Hierarchy Process (AHP) technique (Park \& Jung, 1996)(Park \& Jung, 1996)(Park \& Jung, 1996)(Park \& Jung, 1996)(Park \& Jung, 1996). AHP is used to determine the relative importance of a set of factors by pair wise comparison.

According to ElMaraghy et al. (2008), it is recommended that a mapping function is used to scale the HEP in the range of $10^{-6}$ and 1 . This scaling is performed using the following function: 


$$
\log _{10}(H E P)=6 \times \log _{10}(U(l, f))
$$

To illustrate the effect of input parameters on the error rate in equation (6.5) in numerical examples the input parameters are set as follow:

- Total forgetting time is set at $B=1$ year.

- The relative contribution of learning and fatigue to the error rate is considered as follow:

- $w_{l}=1 / 3$ and $w_{f}=2 / 3$, where learning is less important than fatigue,

- $w_{l}=1 / 2$ and $w_{f}=1 / 2$, where learning and fatigue are equally important,

- $w_{l}=2 / 3$ and $w_{f}=1 / 3$, where learning is more important than fatigue.

- The initial time to perform the task is set as $T_{1}=4$ minutes.

- The learning rate is set at 3 levels corresponding to the slow (90\%), moderate (80\%), and fast (70\%) learning with $b=0.152, b=0.322$, and $b=0.515$, respectively (Dar-El et al., 1995).

- The fatigue accumulation rate is set at 3 levels corresponding to the slow, moderate, and fast fatigue accumulation with $\lambda=6.4 \times 10^{-3}, \lambda=9.6 \times 10^{-3}$, and $\lambda=0.19$, respectively.

- The recovery rate is set to 3 levels corresponding to the slow, moderate, and fast recovery with $\mu=6.4 \times 10^{-3}, \mu=9.6 \times 10^{-3}$, and $\mu=0.19$, respectively.

The fatigue accumulation index and the recovery index were determined using a test. With the assumption of $99 \%$ fatigue at the end of an 8 hour work shift, the fatigue index is obtained as $9.6 \times 10^{-3}$ for the medium fatigue accumulation level from (4.7). The same assumption applies to obtain the indices for the cases of slow and fast fatigue accumulation where $99 \%$ fatigue is 
assumed to be reached in 4 and 12 hours respectively, which result to $\lambda=0.19$ and $\lambda=6.4 \times 10^{-3}$ respectively. The same procedure was used to determine the recovery indices with the assumption of $99 \%$ recovery at the end of a 4,8 , and 12 hours rest break. The recovery indices are obtained as $\lambda=0.19, \mu=9.6 \times 10^{-3}$, and $\mu=6.4 \times 10^{-3}$ from equation (4.9) respectively.

The human error is estimated over a work-break schedule taken from Ihara (2007) for a Toyota assembly line as presented in Table 6.2. The total time for the working period is considered as 7.58 hours including two breaks of lengths 10 minutes and one break of length 45 minutes.

Table 6.2. Work schedule in a shift with four working cycles adding up to 455 minutes.

\begin{tabular}{|c|c|c|}
\hline Working hours & Cycle time $(\mathrm{min})$ & Rest time (min) \\
\hline $6: 25$ a.m- $8: 30$ a.m & 125 & 10 \\
\hline $8: 40$ a.m- $10: 40$ a.m & 120 & 45 (lunch break) \\
\hline 11:25 a.m- 1:25 p.m & 120 & 10 \\
\hline 1:35 p.m- 3:05 p.m & 90 & -- \\
\hline 7.58 (hrs) & 455 (min) & 65 (min) \\
\hline
\end{tabular}

Table 6.3. Parameters' initial values.

\begin{tabular}{lll}
\hline Description of the parameter & Symbol & Numerical values \\
\hline Learning index & $b$ & 0.322 \\
Time to produce the first unit & $T_{1}$ & 4 minutes \\
Total forgetting time & $B$ & 1 year \\
Fatigue index & $\lambda$ & $9.6 \times 10^{-3}$ \\
Recovery index & $\mu$ & $9.6 \times 10^{-3}$ \\
Break time & $\tau$ & $10,45,10$ minutes \\
Learning to Fatigue weight ratio & $w_{l}, w_{f}$ & 1 \\
\hline
\end{tabular}

\subsection{Numerical examples and results}

In this section, numerical analyses are provided to demonstrate the model behavior. A base model is created using the medium values of the problem parameters and additional insights are gained through six research questions. The work-rest schedule in Table 6.2 and 


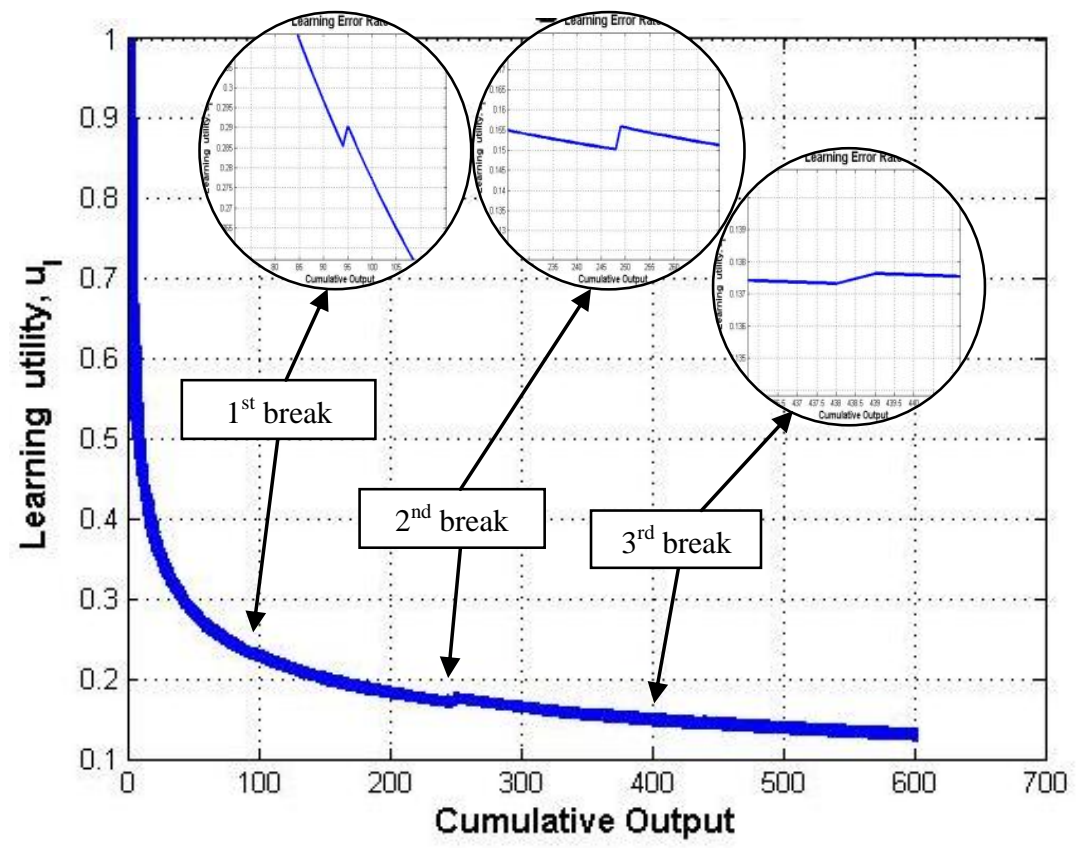

Figure 6.1. The learning error utility function over the working shift with small rises over breaks.

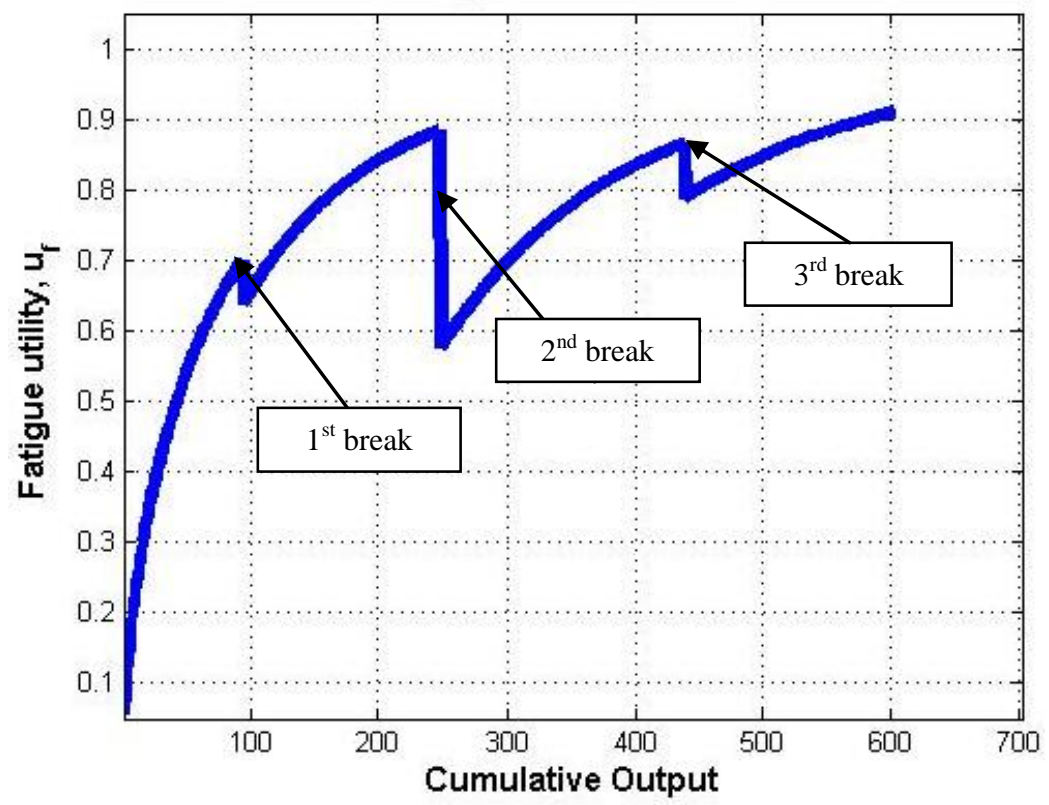

Figure 6.2. The fatigue error utility function over the working shift with declines over breaks.

parameters value in Table 6.3 are used to obtain the base error rate. The learning and fatigue utility (error percentage) functions from equation (6.2) and equation (6.4) over the shift are 
obtained as in Figure 6.1 and Figure 6.2. Figure 6.1 shows that the first utility function associated with error (learning induced error) declines (from 1 to 0.13 ) with accumulated experience and increases very slightly over the breaks. Figure 6.2 shows that the second utility function associated with error (fatigue induced error) increases (from 0 to 0.91 ) with cumulative output and decreases slightly over the breaks.

The behaviour of HEP in equation (6.6) is illustrated in Figure 6.3. In this figure, the variation of error rate with time is presented by the blue line and with cumulative output with red line. The dotted line suggests that during the first working cycle, the error rate reduces because of learning. In this cycle, the error rate reaches its minimum of 0.0017 after 11 repetitions and then starts to increase up to the point when production ceases. During the first break, the worker forgets some of the experience he/she just gained, but also gets some rest, therefore, the error probability decreases. In the second working shift, the error rate increases to its maximum value of 0.021. During the second break, the error rate drops to 0.003 . After the second break, the error rate becomes less sensitive to changes in learning and more sensitive to fatigue, which begins to dominate. The third working shift ends with an error rate of 0.016 , which drops to 0.01 during the last break. In the fourth working cycle the error rate increases up to 0.02 .

The results presented in Figure 6.3 show that the human error rate varies between 0.002 and 0.022 with the highest being 0.022 at the end of the second working cycle and second highest of 0.02 at the end of the fourth. The average error rate probability due to learning and fatigue (and their counter functions, forgetting and recovery) therefore, is 0.012 . Figure 6.3 also demonstrates that the error probability increases with the cumulative output in all working cycles except for 
the first cycle while the effect of learning dominates. Now the behavior of the developed model is examined against the following research questions:

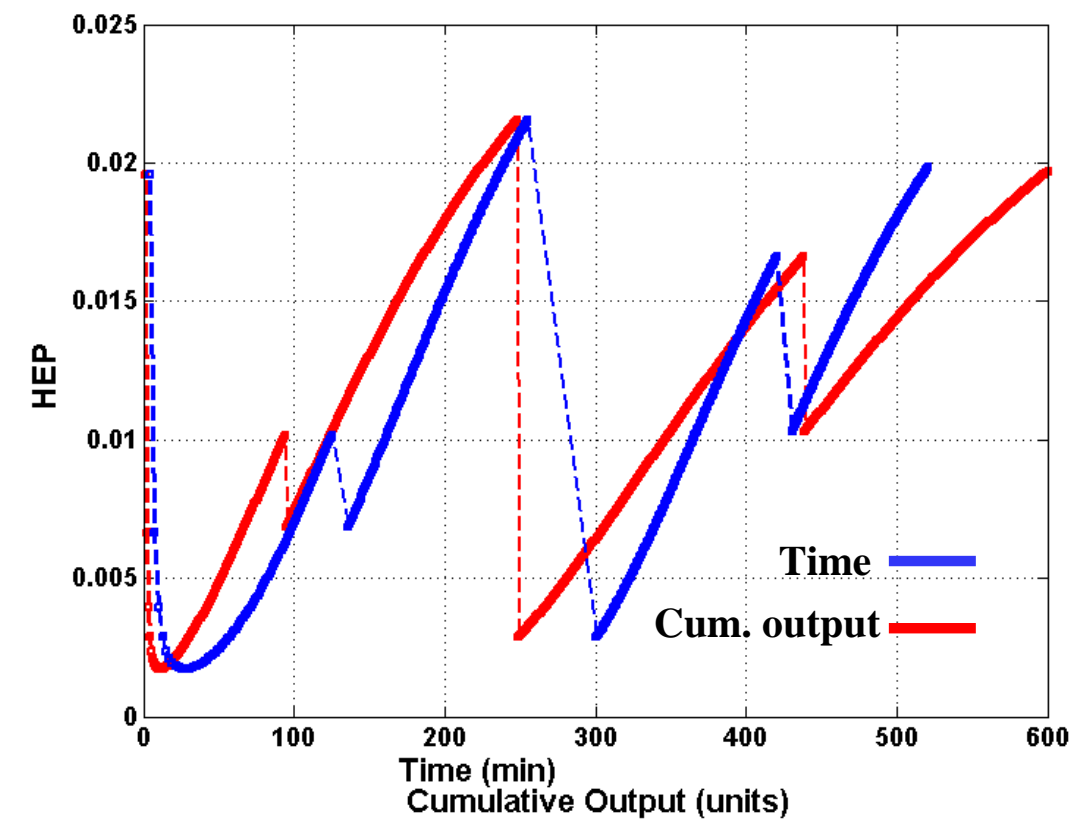

Figure 6.3. Human error probability (HEP) with learning-forgetting and fatigue-recovery over the working shift, downward phases are the breaks.

RQ 1- What effect do the learning, fatigue, and recovery indices have on the human error?

RQ 2- What effect does changing the weighting of learning and fatigue in equation (6.5) have on the HEP results?

RQ 3- What work-rest schedule yields the highest process throughput?

RQ 4- What work-rest schedule results in the lowest worker fatigue?

RQ 5- What is the effect of additional ( $5 \mathrm{~min}$ ) break times on the optimized process in questions 3 and 4 ?

RQ 6- Are the measures of HRA used here comparable with other HRA methods? 
RQ 1- Effects of Learning, fatigue and recovery indices- To examine this question, one parameter is changed at a time and the other two parameters are kept at their medium values (as per Table 6.3). HEP is estimated from equation (6.6) for each working cycle and averaged over the shift. The results in Table 6.4 to Table 6.6 summarise the contribution of learning, fatigue, and recovery to the generation of the human error probability and examines the first research question. For example, to observe the effect of learning, the learning index varies from slow to fast learning rates while fatigue and recovery indices remain at their medium value. The results in Table 6.4 show that the average probability of making a mistake on the assembly line decreases as learning becomes faster. That is, a worker with a fast learning (70\%) tends to make fewer mistakes than a learner with a slow learning (of 90\%), i.e., HEP is less. The results in Table 6.5 show the contribution of fatigue to the generation of errors, where the fatigue accumulation is described as slow, medium, and fast corresponding to the task demands (MacDonald, 2003), i.e., the more fatiguing the task, the more demanding it is and has higher HEP values. The results in Table 6.6 show the effect of recovery on the human error probability, where slow recovery, causes less fatigue to be alleviated during the breaks and more residual fatigue is carried by the worker to the forthcoming working cycles. In this case, the error probability is the highest $(0.014)$. As faster recovery is achieved during the breaks, the error probability decreases to 0.012 and 0.002 for the cases of medium and fast recovery respectively. The results of Table 6.4 to Table 6.6 show that the human error probability and related defects decrease with faster learning, slower fatigue accumulation, and faster recovery which answer RQ 1.

To examine research questions 2 to 5 , a base case scenario is generated. Using medium values of the problem parameters as per Table 6.3, a worker produces 600 units in a work shift, resulting to 
an average fatigue of $83.97 \%$, average human error probability of 0.012 , and 7.2 defective units, equivalent to a throughput of $98.8 \%((600-7.2) / 600)$ as shown in Table 6.7.

Table 6.4. Average human error probability for different learning indices, fatigue and recovery indices are set at medium values.

\begin{tabular}{|l|c|c|c|}
\hline \multicolumn{1}{|c|}{ Learning rate } & $b$ & $\overline{H E P}$ & $\begin{array}{c}\text { Defects } \\
\text { (units/shift) }\end{array}$ \\
\hline Slow (90\%) & 0.152 & 0.015 & 9 \\
\hline $\begin{array}{l}\text { Medium (80\%) } \\
\text { (base) }\end{array}$ & 0.322 & 0.012 & 7.2 \\
\hline Fast $(70 \%)$ & 0.515 & 0.008 & 4.8 \\
\hline
\end{tabular}

Table 6.5. Average human error probability for different fatigue accumulation indices, learning and recovery indices are set at medium values.

\begin{tabular}{|l|c|c|c|}
\hline $\begin{array}{c}\text { Fatigue } \\
\text { accumulation }\end{array}$ & $\lambda$ & $\overline{\text { HEP }}$ & $\begin{array}{c}\text { Defects } \\
\text { (units/shift) }\end{array}$ \\
\hline Slow & $6.4 \times 10^{-3}$ & 0.008 & 4.8 \\
\hline Medium (base) & $9.6 \times 10^{-3}$ & 0.012 & 7.2 \\
\hline Fast & 0.19 & 0.020 & 12 \\
\hline
\end{tabular}

Table 6.6. Average human error probability for different recovery indices, learning and fatigue accumulation indices are set at medium values.

\begin{tabular}{|l|c|c|c|}
\hline \multicolumn{1}{|c|}{ Recovery } & $\mu$ & $\overline{\text { HEP }}$ & $\begin{array}{c}\text { Defects } \\
\text { (units/shift) }\end{array}$ \\
\hline Slow & $6.4 \times 10^{-3}$ & 0.014 & 8.4 \\
\hline Medium (base) & $9.6 \times 10^{-3}$ & 0.012 & 7.2 \\
\hline Fast & 0.19 & 0.002 & 1.2 \\
\hline
\end{tabular}

Table 6.7. The results of the base case (production of 600 units over a 455 minutes work shift).

\begin{tabular}{|c|c|c|c|c|}
\hline Tot. prod. & $\begin{array}{c}\text { Max Fatigue } \\
(\%)\end{array}$ & $\begin{array}{c}\overline{\mathrm{HEP}} \\
\text { (average human } \\
\text { error probability) }\end{array}$ & $\begin{array}{c}\mathrm{DEF} \\
\text { (defective items) }\end{array}$ & $\begin{array}{c}T P \\
\text { (throughput) }\end{array}$ \\
\hline 600 & 83.97 & 0.012 & 7.2 & 98.8 \\
\hline
\end{tabular}

RQ 2- Effect of learning and fatigue weights on the throughput- The structure of RQ 2 is shown in Table 6.8 and the results are compared with the base case in Table 6.9 to investigate 
how the different combinations of learning and fatigue weights, affect the process throughput. According to the results, learning decreases the human error rate while fatigue increases it. Therefore, if the decision maker gives more attention to learning (by choosing a greater weight for learning in $\left.(6.5) ; w_{l}>w_{f}\right)$, HEP is minimum and the throughput is more than the average (i.e. the base case). This case is presented by RQ 2-I in Table 6.9. The opposite was observed when the decision maker gives more weight to fatigue in producing error $\left(w_{l}<w_{f}\right)$. In this case the error rate is high, and the throughput is less than the average. This case is presented by RQ 2II in Table 6.9. Given the conditions that govern the working environment, and according to the available data from the firm, it is up to the user to specify the proper weights when using equation (6.5).

Table 6.8. Weight setting for maximum throughput, problem statement.

\begin{tabular}{|l|l|}
\hline \multicolumn{2}{|c|}{$\mathrm{RQ} 2$} \\
\hline Objective Function & Throughput $=\frac{\text { No.of outputs }- \text { No.of defectives }}{\text { No.of outputs }}$ \\
\hline Effective parameters & Learning weight $\boldsymbol{w}_{l}$, fatigue weight $\boldsymbol{w}_{f}$ \\
\hline
\end{tabular}

Table 6.9. Weight setting for maximum throughput, solution.

\begin{tabular}{|c|c|c|c|c|c|c|}
\hline & $\boldsymbol{w}_{\boldsymbol{l}}, \boldsymbol{w}_{\boldsymbol{f}}$ & $\begin{array}{c}\text { Tot. } \\
\text { production }\end{array}$ & $\begin{array}{c}\text { Max Fatigue } \\
(\%)\end{array}$ & $\begin{array}{c}\overline{\mathrm{HEP}} \\
\text { (ave. hum. err. } \\
\text { prob.) }\end{array}$ & $\begin{array}{c}\text { DEF } \\
\text { (def. items) }\end{array}$ & $\begin{array}{c}\text { TP } \\
\text { (throughput) }\end{array}$ \\
\hline Base Case & $0.5,0.5$ & 600 & 83.97 & 0.012 & 7.2 & 98.8 \\
\hline RQ 2- I & $0.9,0.1$ & 600 & 83.97 & 0.002 & 1.2 & 99.8 \\
\hline RQ 2- II & $0.1,0.9$ & 600 & 83.97 & 0.153 & 91.8 & 84.7 \\
\hline
\end{tabular}


RQ 3- The work-rest schedule that yields the highest process throughput- The aim is to examine the optimum working schedule for maximum throughput based on the 3 breaks per shift. In the current schedule, 600 items, $\sum x_{i}$, are produced in 455 minutes, $\sum t_{i}$, and workers are given a total break time, $\sum \tau_{i}$, of 65 minutes. The optimization problem is defined according to Table 6.10 where the throughput is maximized for a total production of more than 600 units, $\sum x_{i} \geq 600$. Also it is assumed that each working period is more than 60 minutes and less than 125 minutes, $60 \leq t_{i} \leq 125$, the total working time is less than 455 minutes, $\sum t_{i} \leq 455$, and each break time is more than 5 minutes $\left(\tau_{i} \geq 5 \mathrm{~min}\right)$. The optimized schedule in Table 6.11 (case RQ 3) indicates that the optimized cycles times of $122,120,118$, and 95 minutes are very close to the current times of $125,120,120$, and 90 minutes in the base case. The present working shift (base case), yields a throughput of $98.87 \%$ while optimized model shows a $0.02 \%$ increase in the throughput, which can be obtained by reducing fatigue through small changes in break times.

RQ 4- The work-rest schedule that yields the lowest worker fatigue. The mathematical programming model for RQ 4 is presented in Table 6.12 with the results summarized in Table 6.13. Results in Table 6.13 indicate that the redistribution of break times $(10,27.5,27.5)$ instead of $10,45,10)$ improves the system's throughput and it is associated with less fatigue $(83.97 \rightarrow$ $79.54)$ and fewer defective items $(6.78 \rightarrow 6.14)$. However, implementing a break of 27.5 minutes may not be practical to measure, so perhaps a closer schedule to the indicated scenario as of 10 , 25 , and 30 minutes seems to be more practical.

RQ 5- Effect of additional break time on the optimal schedule obtained in RQ 3 and 4 . In RQ-5 the total break time increases by 5 minutes and the maximum fatigue and throughput are 
compared with the base case. Therefore, workers are given a total break time between 65 and 70 minutes, $65 \leq \sum \tau_{i} \leq 70$. Two optimization problems are defined. The first problem is defined in Table 6.14 where workers are given 5 minutes additional break without any other constraint over the distribution of break times. The solution for this problem is presented in Table 6.15 with two optimization cases, maximizing the throughput (RQ 5-I) and minimizing fatigue (RQ 5-II) by cycle time and breaks, with the constraints of a total production of more than 600 units, and the same total cycle time of 455 minutes. In the second problem, that is defined in Table 6.16, workers are given 5 minutes additional break time, with a constraint that the lunch break is more than 30 minutes, $\tau_{2} \geq 30$. The solution for this problem is found in Table 6.17 for maximizing the throughput (RQ 5-III) and minimizing the fatigue (RQ 5-IV) with the same previous constraints for time and production.

Case RQ 5-I in Table 6.15 indicates that giving slightly longer breaks to the workers (70 rather than $65 \mathrm{~min})$ improves the system's throughput $(98.87 \rightarrow 98.95)$ associated with less fatigue $(83.97 \rightarrow 83.11)$ and less number of defective items $(6.78 \rightarrow 6.24)$. The optimized schedule for maximum throughput in this case is suggested to be $125,125,90$, and 115 minutes for working times and the break schedule to be 10,35 , and 25 minutes.

Case RQ 5-II in Table 6.15 shows that giving slightly longer breaks to the workers (70 rather than $65 \mathrm{~min})$ improves the system's throughput $(98.87 \rightarrow 98.99)$ associated with less fatigue $(83.97 \rightarrow 79.37)$ and less number of defective items $(6.78 \rightarrow 6.06)$. The optimized schedule for minimum fatigue in this case is suggested to be $80,125,125$, and 125 minutes for working times and the break schedule to be 10,20 , and 40 minutes. 
Table 6.10. Schedule optimization for maximum throughput, problem statement.

\begin{tabular}{|l|l|}
\hline RQ 3 & Schedule Optimization for max throughput \\
\hline Objective Function & Throughput $=\frac{\text { No.of outputs }- \text { No. of defectives }}{\text { No.of outputs }}$, to be maximized \\
\hline With respect to & Cycle time and breaks \\
\hline Constraints & $\sum x_{i} \geq 600, \sum t_{i} \leq 455,60 \leq t_{i} \leq 125, \sum \tau_{i} \geq 65, \tau_{i} \geq 5$ \\
\hline
\end{tabular}

Table 6.11. Schedule optimization for maximum throughput, solution.

\begin{tabular}{|c|c|c|c|c|c|c|c|}
\hline & Cycle time & Tot. prod. & $\begin{array}{c}\text { Max Fatig. } \\
(\%)\end{array}$ & Breaks & $\begin{array}{c}\overline{H E P} \\
\text { (ave. hum. err. } \\
\text { prob.) }\end{array}$ & $\begin{array}{c}\text { DEF } \\
\text { (def. items) }\end{array}$ & $\begin{array}{c}\text { TP } \\
\text { (throughput) }\end{array}$ \\
\hline $\begin{array}{c}\text { Base } \\
\text { Case }\end{array}$ & $\begin{array}{c}125,120,120, \\
90 \\
(455 \mathrm{~min})\end{array}$ & 600 & 83.97 & $\begin{array}{c}10,45,10 \\
(65 \mathrm{~min})\end{array}$ & 0.011 & 6.78 & 98.87 \\
\hline RQ 3 & $\begin{array}{c}122,120,118, \\
95 \\
(455 \mathrm{~min})\end{array}$ & 600 & 83.67 & $\begin{array}{c}12,40,13 \\
(65 \mathrm{~min})\end{array}$ & 0.011 & 6.64 & 98.89 \\
\hline
\end{tabular}

Table 6.12. Schedule optimization for minimum fatigue, problem statement.

\begin{tabular}{|l|l|}
\hline RQ 4 & Schedule optimization for minimum fatigue \\
\hline Objective Function & Fatigue, to be minimized \\
\hline With respect to & Cycle time and rest breaks \\
\hline Constraints & $\sum x_{i} \geq 600, \sum t_{i} \leq 455,60 \leq t_{i} \leq 125, \sum \tau_{i} \geq 65, \tau_{i} \geq 5$ \\
\hline
\end{tabular}

Table 6.13. Schedule optimization for minimum fatigue, solution.

\begin{tabular}{|l|c|c|c|c|c|c|c|}
\hline & Cycle time & Tot. prod. & $\begin{array}{c}\text { Ave. Fatig. } \\
(\%)\end{array}$ & Breaks & $\begin{array}{c}\overline{\text { HEP }} \\
\text { (ave. hum. } \\
\text { prob. })\end{array}$ & $\begin{array}{c}\text { DEF } \\
\text { (def. items) }\end{array}$ & $\begin{array}{c}\text { TP } \\
\text { (throughput) }\end{array}$ \\
\hline $\begin{array}{c}\text { Base } \\
\text { Case }\end{array}$ & $\begin{array}{c}125,120,120, \\
90 \\
(455 \mathrm{~min})\end{array}$ & 600 & 83.97 & $\begin{array}{c}10,45,10 \\
(65 \mathrm{~min})\end{array}$ & 0.011 & 6.78 & 98.87 \\
\hline RQ 4 & $\begin{array}{c}80,125,125, \\
125 \\
(455 \mathrm{~min})\end{array}$ & 600 & 79.54 & $\begin{array}{c}10,27.5,27.5 \\
(65 \mathrm{~min})\end{array}$ & 0.0102 & 6.14 & 98.97 \\
\hline
\end{tabular}


Table 6.14. Effect of additional break time on optimal schedules, problem statement.

\begin{tabular}{|l|l|}
\hline RQ 5 & Effect of additional break time on optimal schedules \\
\hline Objective Function & $\begin{array}{l}\text { Throughput (I), to be maximized } \\
\text { Fatigue (II), to be minimized }\end{array}$ \\
\hline With respect to & Cycle time and rest breaks \\
\hline Constraints & $\sum x_{i} \geq 600, \sum t_{i} \leq 455,60 \leq t_{i} \leq 125,65 \leq \sum \tau_{i} \leq 70, \tau_{i} \geq 5$ \\
\hline
\end{tabular}

Table 6.15. Effect of additional break time on optimal schedules, solution.

\begin{tabular}{|c|c|c|c|c|c|c|c|}
\hline & Cycle time & Tot. prod. & $\begin{array}{c}\text { Ave. Fatig. } \\
(\%)\end{array}$ & Breaks & $\begin{array}{c}\overline{\text { HEP }} \\
\text { (ave. hum. err. } \\
\text { prob.) }\end{array}$ & $\begin{array}{c}\text { DEF } \\
\text { (def. items) }\end{array}$ & $\begin{array}{c}\text { TP } \\
\text { (throughput) }\end{array}$ \\
\hline Base Case & $\begin{array}{c}125,120, \\
120,90 \\
(455 \mathrm{~min})\end{array}$ & 600 & 83.97 & $\begin{array}{c}10,45,10 \\
(65 \mathrm{~min})\end{array}$ & 0.011 & 6.78 & 98.87 \\
\hline $\begin{array}{c}\text { RQ 5-I } \\
\text { Max TP }\end{array}$ & $\begin{array}{c}125,125,90, \\
115 \\
(455 \mathrm{~min})\end{array}$ & 600 & 83.11 & $\begin{array}{c}10,35,25 \\
(\mathbf{7 0} \mathrm{min})\end{array}$ & 0.0104 & 6.25 & 98.95 \\
\hline $\begin{array}{c}\text { RQ 5-II } \\
\text { min fatigue }\end{array}$ & $\begin{array}{c}80,125,125, \\
125 \\
(455 \mathrm{~min})\end{array}$ & 600 & 79.37 & $\begin{array}{c}10,20,40 \\
(\mathbf{7 0} \text { min })\end{array}$ & 0.0101 & 6.06 & 98.99 \\
\hline
\end{tabular}


Table 6.16. Effect of additional break time on optimal schedules, lunch break > 30 minutes, problem statement.

\begin{tabular}{|l|l|}
\hline RQ 5 & $\begin{array}{l}\text { Effect of additional break time on optimal schedules and a lunch break } \\
\text { longer than } 30 \text { minutes }\end{array}$ \\
\hline Objective Function & $\begin{array}{l}\text { Throughout (III), to be maximized } \\
\text { Fatigue (IV), to be minimized }\end{array}$ \\
\hline With respect to & Cycle time and rest breaks \\
\hline Constraints & $\sum x_{i} \geq 600, \sum t_{i} \leq 455,60 \leq t_{i} \leq 125,65 \leq \sum \tau_{i} \leq 70, \tau_{i} \geq 5, \tau_{2} \geq 30$ \\
\hline
\end{tabular}

Table 6.17. Effect of additional break time on optimal schedules, lunch break > 30 minutes, solution.

\begin{tabular}{|c|c|c|c|c|c|c|c|}
\hline & Cycle time & Tot. prod. & $\begin{array}{c}\text { Ave. Fatig. } \\
(\%)\end{array}$ & Breaks & $\begin{array}{c}\overline{H E P} \\
\text { (ave. hum. err. } \\
\text { prob.) }\end{array}$ & $\begin{array}{c}\text { DEF } \\
\text { (def. items) }\end{array}$ & $\begin{array}{c}\text { TP } \\
\text { (throughput) }\end{array}$ \\
\hline Base Case & $\begin{array}{c}125,120, \\
120,90 \\
(455 \mathrm{~min})\end{array}$ & 600 & 83.97 & $\begin{array}{c}10,45,10 \\
(65 \mathrm{~min})\end{array}$ & 0.011 & 6.78 & 98.87 \\
\hline $\begin{array}{c}\text { RQ 5-III } \\
\text { Max TP }\end{array}$ & $\begin{array}{c}125,125,90, \\
115 \\
(455 \mathrm{~min})\end{array}$ & 600 & 83.11 & $\begin{array}{c}10,35,25 \\
(\mathbf{7 0} \mathrm{min})\end{array}$ & 0.0104 & 6.25 & 98.95 \\
\hline $\begin{array}{c}\text { RQ 5-IV } \\
\text { min fatigue }\end{array}$ & $\begin{array}{c}100,125, \\
125,105 \\
(455 \mathrm{~min})\end{array}$ & 600 & 81.35 & $\begin{array}{c}10,30,30 \\
(\mathbf{7 0} \text { min })\end{array}$ & 0.0102 & 6.13 & 98.98 \\
\hline
\end{tabular}

Case RQ 5-III in Table 6.17 indicates that giving 5 minutes longer breaks to the workers (70 rather than $65 \mathrm{~min}$ ) and imposing a lunch break longer than 30 minutes, improves the system's throughput $(98.87 \rightarrow 98.95)$ associated with less fatigue $(83.97 \rightarrow 83.11)$ and less number of defective items $(6.78 \rightarrow 6.25)$. The optimized schedule for maximum throughput in this case is suggested to be $125,125,90$, and 115 minutes for working times and the break schedule to be 10,35 , and 25 minutes.

Case RQ 5-IV in Table 6.17 shows that 5 minutes additional break time and a lunch break longer than 30 minutes $(70$ rather than $65 \mathrm{~min})$ improves the system's throughput $(98.87 \rightarrow 98.98)$ 
associated with less fatigue $(83.97 \rightarrow 81.35)$ and less number of defective items $(6.78 \rightarrow 6.13)$. The optimized schedule for minimum fatigue in this case is suggested to be $100,125,125$, and 105 minutes for working times and the break schedule to be 10, 30, and 30 minutes.

The results in Table 6.15 and Table 6.17 indicate that having an extra constraint for lunch breaks ( $\tau_{2} \geq 30$ ) does not affect the throughput of the system. This is because cases RQ 5-I and RQ 5III both have the same work-cycle times $(125,125,90$, and 115) and an optimal lunch break of more than 30 minutes ( $\tau_{2}=35$ minutes). However, applying a lunch break more than 30 minutes results to less throughput and more fatigue in case RQ 5-IV when compared to case RQ 5-II. This is because the work-cycle times of $80,125,125$, and 125 with a 20 minutes lunch break in case RQ 5-II, generate higher throughput and less fatigue when compared to $100,125,125$, and 105 work-cycles with a 30 minutes lunch break for case RQ 5-IV. This case is an example of how workers' welfare may be ignored in an exchange for higher throughput. Having a lunch break of 30 minutes seems reasonable for workers while managers may be more willing to give them a lunch break of 20 minutes for better throughput. These results suggest that a break time between 20-30 minutes may be agreeable for both parties.

RQ 6- How measured HEP of this model compares to other methods - average HEPs obtained by this method $(\overline{H E P})$ are compared with HEART (Williams, 1986) in two extreme cases of best and worst. The best case, corresponds to the minimum $\overline{H E P}$, is obtained by having a fast learning, slow fatigue accumulation, and fast recovery from fatigue as 0.0014 . In the worst case, the maximum $\overline{H E P}$, is obtained as 0.028 by slow learning, fast fatigue accumulation, and slow recovery. The results are presented in Table 6.18 . 
Table 6.18. The best case and worst case scenarios representing the spectrum of human error probability of the presented model.

\begin{tabular}{|c|c|c|c|c|c|c|c|c|c|}
\hline \multirow[t]{2}{*}{ Scenario } & \multirow[b]{2}{*}{ learning } & \multirow[b]{2}{*}{ fatigue } & \multirow[b]{2}{*}{ recovery } & \multicolumn{4}{|c|}{$\overline{H E P}_{i}$} & \multirow{2}{*}{$\begin{array}{c}\overline{H E P} \\
\text { Total } \\
\text { averag } \\
e\end{array}$} & \multirow{2}{*}{$\begin{array}{c}\text { HEART } \\
H E P=0.02\end{array}$} \\
\hline & & & & $\begin{array}{c}I^{s t} \\
\text { cycle }\end{array}$ & $\begin{array}{c}2^{\text {nd }} \\
\text { cycle }\end{array}$ & $\begin{array}{c}3^{\text {rd }} \\
\text { cycle }\end{array}$ & $\begin{array}{c}4^{\text {th }} \\
\text { cycle }\end{array}$ & & \\
\hline Best case & Fast & slow & fast & 0.0034 & 0.001 & 0.0009 & 0.0004 & 0.0014 & $\begin{array}{l}\text { HEART } \\
\text { overestimates HEP }\end{array}$ \\
\hline Worst case & Slow & fast & slow & 0.007 & 0.05 & 0.023 & 0.035 & 0.028 & $\begin{array}{l}\text { HEART } \\
\text { underestimates HEP }\end{array}$ \\
\hline
\end{tabular}

for the best case scenario, $\overline{H E P}=0.0014$, is $93 \%$ less than HEART's estimation for a similar task (Smith, 2011). For the worst case, $\overline{H E P}=0.028$, is $40 \%$ more than HEART's prediction. The average HEP obtained by this method is 0.0147 , which is $26 \%$ less than the HEART method. These results suggest that unlike HEART, our model is more responsive to the variation of work condition and worker abilities.

\subsection{Discussion and conclusion}

Human is central for manufacturing facilities that seek workforce flexibility. There are many manufacturing jobs that require human involvement (Tang et al., 2003), especially in the automation gaps, where the operator is left with tasks that have not been automated (Luczak \& Mueller, 1994). Humans are prone to commit mistakes for many reasons relating to the work environment, which results in defective units that should be dealt with at a cost. The common problem with available HRA methods is that they do not tell when and how an error happens. The effect of PSFs in these models is included by non-varying measures that do not change during a routine job. Furthermore, in these models, the PSFs such as learning and fatigue are not 
usually considered. The model developed in this Phase is a function of time which allows for estimating the probability of error, and subsequently the number of defective units over a working shift. It captures the effect of learning and fatigue accumulation on a job. The developed model estimates the probability of committing error on a job at any specific time in a work shift and demonstrates that the human error probability and related defects decrease with faster learning, slower fatigue accumulation, and faster recovery. This result may help in setting inspection and maintenance plans that are based on the times of the working shift when the error rate is expected to be relatively high. Furthermore, the model could provide optimized schedules to increase throughput or decrease the worker's fatigue, however, the improvement scale depends on the process costs and also product value. Here, within the considered range of parameters, and without changing the base work-rest schedule significantly, the throughput could be improved by $0.12 \%$ by decreasing the defect rate through less fatigue.

The results of this research could simplify the modelling of HRA models by only considering the factors that significantly affect human performance, i.e. learning and fatigue. To decrease the error rate, it is recommended to design jobs where workers acquire more learning during the process. Tang et al. (2003) observed that the error rate of an assembly task could be reduced up to $82 \%$ by using methods to educate workers about their jobs such as overlaying 3D instructions on the actual work pieces. Designing more ergonomic workstations also results in less fatigue on a job and decreases human error (Yeow \& Nath Sen, 2003; Molaoa, 2008).

Phase 3 of this thesis contradicts the traditional managerial attitude to boost the process outcome by shrinking the breaks and increasing the working times. As results suggested, excessive fatigue as a result of less break times, has adverse effect on the products quality and process outcomes, 
not to mention the related injuries and insurance costs. Conversely, results indicate that more break times, improve the process throughput by less mistakes and higher quality products. 


\section{Phase 4: Investigating flexibility and transfer policies in a DRC system with some human factors}

The aim of this Phase of the thesis is to present a DRC system model where the human contributes to the performance of the system and quality of the final product. Using the Learning Forgetting Fatigue Recovery Model (LFFRM) of Phase 1 and Quality Learning Forgetting Curve (QLFC) of Phase 2, and the product quality model of Phase 3, the system performance with a cost function is examined. The results are used to determine the production planning and lot sizing policy in a DRC system.

\subsection{Introduction}

Dual-Resource Constrained (DRC) systems are the working environments where the number of workers is less than the number of machines or workstations (Zamiska et al., 2007). Workers in a DRC system are cross-trained to acquire several skills that increase their flexibility and allow them to perform a variety of tasks (Thannimalai et al., 2013). Cross-trained workers usually handle the situations of unexpected orders and unbalanced workloads. A flexible workforce helps reduce lead times and improve customer service (Nembhard et al., 2002; Bokhorst \& Gaalman, 2009), however, a full cross-trained workforce may not be feasible due to either the training costs, required specific skills or equipment (Gel et al., 2007; Robbins et al., 2007). In labor-intensive environments, workers accumulate experience (learning) through repetitions but, as soon as they cease the repetitions, in order to take a break or transfer to another job, they start to forget their previous learning or experience. Also, while performing a job, a worker accumulates fatigue, which must be recovered by rest breaks or transferring to a less demanding job. The performance of DRC systems improves while workers learn, but is impeded when 
workers forget their skills and knowledge. Also, the performance of the workers declines with fatigue and improves with recovery. Therefore, the intermittent cycles of learning-fatigue and forgetting-recovery have adverse effects on the system performance. While the performance of DRC systems improves with the flexibility of workers (Jaber et al., 2003; Azizi et al., 2010; Jahandideh, 2012), alternating between different jobs or going on a break impedes system performance and influences process quality. In DRC systems, learning and forgetting, fatiguerecovery, and quality have been studied separately, but to the best knowledge of the authors, there is no study that captures the combined effects of these phenomena. The focus of this Phase is industrial settings where workers perform tasks that require them to identify and select the component and follow a sequence to assemble it (Jaber \& Kher, 2002). In this process, learning occurs during the assembly process and forgetting occurs when workers shift back and forth between different assembly stations or products. The present Phase of the thesis, contributes to the DRC system literature by presenting a production planning model that captures the aspects of human behaviour such as learning, forgetting, fatigue, and recovery, as well as error making and creates production schedules that are more realistic and applicable to such working environment. The rest of this section is organized as follows: Section 7.2 presents the methodology of this Phase. Section 7.3 presents the results and Section 7.4 discusses and concludes this Phase.

\subsection{Methodology}

The LFFRM developed in Section 4, QLFC developed in Section 5, and the HRA model developed in 6 are used in this Section to develop a comprehensive model of a DRC system with human effects. The model is then utilized to obtain the optimized policies for production planning and flexibility level. In this Phase of thesis, a production and rework process with 
learning effects is considered to investigate the combined effects of quality, learning-forgetting, and fatigue-recovery on the performance and throughput of DRC systems. It is assumed that the defective items could be generated as a result of human error or when the process goes out of control. Therefore the number of defective units that need reworking is introduced by $d\left(x_{i}\right)$ which is equal to:

$d\left(x_{i}\right)=H E P \times x_{i}+\rho x_{i}^{2} / 2$

in which HEP is human error probability during the production of lot $x_{i}$ (calculated from equation (6.6)) and $\rho x_{i}^{2} / 2$ is the number of defected items in a lot of size $x_{i}$, estimated from Khouja (2005) where $\rho$ is the probability of the process going out of control and producing defective items (from Section 5.3.1). One must be cautious when selecting the value of $\rho$ to produce reasonable values. Khouja (2005) recommend that $\rho$ assumes very small values. Equation (5.10) is rewritten here as:

$T(X)=\sum_{i}\left(Y\left(x_{i}\right)+R\left(x_{i}\right)\right)=\sum_{i}\left(\frac{T_{1}}{1-b}\left[\left(x_{i}+u_{i}\right)^{1-b}-u_{i}^{1-b}\right]+\frac{r_{1}}{1-\varepsilon}\left[\left(d\left(x_{i}\right)+v_{i}\right)^{1-\varepsilon}-v_{i}^{1-\varepsilon}\right]\right)$

In which $T(X)$ is the cumulative time of the process, $Y\left(x_{i}\right)$ is the cumulative production time, and $R\left(x_{i}\right)$ is the cumulative rework time. $r_{1}$ is the time to rework the first unit and term $v_{i}$, similar to $u_{i}$, is the experience (in units) transferred to rework run $i$ from $i-1$ previous runs performed, while $b$ and $\varepsilon$ are the learning indices for the production and rework processes. 
The principle "fixed-before-shared" by Koole and Pot (2006) is used which allows cross-trained workers help their peers after completing their core job, that is, the worker performs the primary job and immediately reworks the defective items. The following assumptions are made in this research:

- The process may shift out-of-control by producing each unit and start to produce defective items with a constant and known probability. Once out-of-control, the process remains in that state until the end of a production run (Porteus, 1986),

- All workers have the same capacity for learning and fatigue accumulation,

- The shop has a constant availability of work,

- No upfront training is provided,

- The rework process is always in control, i.e., no scrap is generated, and

- Defective items are generated as a result of machine malfunction or human error.

- Only one type of job can be performed at each workstation.

\subsubsection{Performance Measure}

Consider a parallel production system in which a worker is assigned to a workstation that produces its own output independently of other workstations (Kim \& Nembhard, 2010). The performance of such a system can be measured by direct and indirect costs of the process. Direct costs relate to the process time, while indirect costs relate to the quality of the products and material consumed during the rework. The following performance measure is proposed:

$$
Z=Z_{1}+Z_{2}=\overbrace{1-\frac{\sum_{i} T_{o p, i}}{\sum_{i} T_{o p, 0}}}^{Z_{1}}+\overbrace{-\frac{\sum_{i} d\left(x_{i}\right)}{\sum_{i} x_{i}}}^{Z_{2}}
$$


in which $Z_{1}$ is the ratio of the sum of production and rework times for all $i$ cycles when considering the learning and forgetting effects, $T_{o p, i}$ and without learning and forgetting, $T_{o p, 0}$. $T_{o p, i}$ is determined from equation (7.2) as $\left[Y\left(x_{i}\right)+R\left(x_{i}\right)\right] / x_{i}$, while $T_{o p, 0}$ is determined in the same manner after substituting $b=\varepsilon=0$. The best performance for $Z_{1}$ and $Z_{2}$ is one and the worst is zero. For example, as the number of reworked items in a cycle approaches a minimum number, i.e. $d\left(x_{i}\right)$ approaches zero, then $\mathrm{Z}_{2}$ approaches 1 .

\subsubsection{Transfer Policies}

Workers transfer between different stations with different levels of workload. Therefore, each workstation is associated with a specific learning and fatigue index, which is represented by the learning and fatigue indices in the following matrices:

$$
\begin{gathered}
b_{w_{m}, s_{j}}=\left[\begin{array}{cccc}
b_{w_{1}, s_{1}} & b_{w_{1}, s_{2}} & \ldots & b_{w_{1}, s_{j}} \\
b_{w_{2}, s_{1}} & \vdots & \vdots & \vdots \\
\vdots & \vdots & \vdots & \vdots \\
b_{w_{m}, s_{1}} & \ldots & \cdots & b_{w_{m}, s_{j}}
\end{array}\right] \\
\lambda_{w_{m}, s_{j}}=\left[\begin{array}{cccc}
\lambda_{w_{1}, s_{1}} & \lambda_{w_{1}, s_{2}} & \ldots & \lambda_{w_{1}, s_{j}} \\
\lambda_{w_{2}, s_{1}} & \vdots & \vdots & \vdots \\
\vdots & \vdots & \vdots & \vdots \\
\lambda_{w_{m}, s_{1}} & \ldots & \ldots & \lambda_{w_{m}, s_{j}}
\end{array}\right]
\end{gathered}
$$

in which, $b_{w_{m}, s_{j}}$ and $\lambda_{w_{m}, s_{j}}$ are the learning and fatigue indices that each worker $m$ experiences at each workstation $j$. By working at each station, the worker accumulates learning and fatigue simultaneously. However, departing from this station, the worker carries away his/her experience and fatigue. By assuming no similarity between workstations, this experience will not become 
useful until the next time the worker visits the same workstation. However, assuming that physical fatigue is only relieved by the rest break, the worker carries along the residual fatigue to the immediate next workstation after the rest break. It should be noted that the rest break, that is usually used to alleviate fatigue and it has been shown by $\tau$ throughout this thesis (equation (4.8)), is now different from the learning break that causes forgetting, that previously was shown by $\varphi$ in LFCM model (equation (4.5)) since they occur independently. Although equations (7.4) and (7.5), represent the general modeling for this system, in this section, we assume that different workers experience the same learning and fatigue while working at the same workstation, i.e., $b_{w_{1}, s_{1}}=b_{w_{2}, s_{1}}=\ldots=b_{w_{m}, s_{1}}$ and $\lambda_{w_{1}, s_{1}}=\lambda_{w_{2}, s_{1}}=\ldots=\lambda_{w_{m}, s_{1}}$ and etc.

\subsubsection{Parameter values}

The values of the parameters are set according to Table 7.1. The learning index is set at 3 levels corresponding to the slow (90\%), moderate (80\%), and fast $(70 \%)$ learning with $b=0.152$, $b=0.322$ and $b=0.515$, respectively (Dar-El et al., 1995). The fatigue index and the recovery index are set as explained in section 6.3. With the assumption of $99 \%$ fatigue at the end of an 8 hour work shift, the fatigue index is obtained as $\lambda=9.6 \times 10^{-3}$ for the medium fatigue accumulation level. The same assumption applies to obtain the indices for the cases of slow and fast fatigue accumulation where $99 \%$ fatigue is assumed to be reached in the 4 and 12 hour work-shift, respectively, which result in $\lambda=0.19$ and $\lambda=6.4 \times 10^{-3}$ respectively. As fatigue accumulation becomes faster, we assume that the work load increases and a task becomes harder, for instance, $\lambda=6.4 \times 10^{-3}$ corresponds to the easiest, $\lambda=9.6 \times 10^{-3}$ to the moderate, and $\lambda=0.19$ to the hardest task. The same procedure applies to find the recovery indices with the assumption 
of $99 \%$ recovery at the end of 4,8 , and 12 hours of rest. The recovery indices were obtained as $\mu=0.19, \mu=9.6 \times 10^{-3}$, and $\mu=6.4 \times 10^{-3}$ for fast, medium, and slow recovery respectively.

Table 7.1. Values of the input parameters used in the numerical study.

\begin{tabular}{|l|c|l|}
\hline Definition & Symbol & \multicolumn{1}{|c|}{ Value(s) } \\
\hline Learning index (fast, moderate, and slow learning) & $b$ & $0.515,0.322,0.152$ \\
\hline Fatigue index (hard, moderate, and easy work) & $\lambda$ & $0.19,9.6 \times 10^{-3}, 6.4 \times 10^{-3}$ \\
\hline Recovery index (fast, moderate, and slow) & $\tau$ & $0.19,9.6 \times 10^{-3}, 6.4 \times 10^{-3}$ \\
\hline Rest break & $T_{l}$ & $15 \mathrm{~min}$ \\
\hline Time to produce the very first unit & $r_{l}$ & $2.5 \mathrm{~min}$ \\
\hline Time to rework the very first unit & $B$ & 1 year $\left(5.26 \times 10^{5} \mathrm{~min}\right)$ \\
\hline Time of total forgetting & $\rho$ & 0.001 \\
\hline Probability of the process goes out of control & $w_{1}, w_{2}$ & $0.5,0.5$ \\
\hline Learning and fatigue weights in error generation & & \multicolumn{1}{c|}{ min } \\
\hline
\end{tabular}

\subsubsection{Scenarios}

This research aims to investigate the performance of DRC systems under the influence of human performance by examining the developed models. Therefore, the system is examined by considering the configuration of workstations, the level of flexibility of the workers, and the number of working cycles in different scenarios.

In each scenario up to 3 workers are assigned to process 9000 jobs. Here, a 'task' means to process one unit in one station per cycle. The rotation plan is applied irrespective of the number of workers, which means, worker 1 transfers from workstation 1 to workstation 2 after processing the required tasks in the first station. If there are two workers, each, transfers to the next workstation after performing assigned tasks. A VBA program is developed to assign workers as well as specific learning and fatigue indices to each station. It also generates a 
rotation plan that transfers the worker to the next workstation after processing his/her share of the lot which is calculated by:

$$
x_{i}=\frac{X}{m \cdot j \cdot c}
$$

in which $x_{i}$ is the share of each worker to process in each cycle, $m$ is the number of workers, $j$ is the number of workstations, and $\mathrm{c}$ is the number of working cycles.

To be eligible to transfer from the current to the next workstation, the worker must process the assigned products and rework any defective item. The learning and fatigue matrices defined in equations (7.4) and (7.5) are assigned to each workstation according to the flexibility level. For example, if the flexibility level is 2 , Flex $=2$, workers transfer between two working stations with two levels of slow and fast learning and fatigue accumulation. If Flex $=3$, there are three working stations and the rates vary in three levels of slow, moderate, and fast. If Flex $=4$, the two middle stations will have the same rates. It should be noted however that the number of workers are always less than or equal to the number of workstations. For instance, if the scenario contains two workstations (Flex $=2$ ), the number of workers can either be 1 or 2 .

Each worker accumulates learning and fatigue while on the job. It is assumed that a number of defective items are generated when processing a batch, which could be caused by many reasons (e.g., fatigue, forgetting, human, and/or process error). After a worker has processed the assigned work and reworked the related defects, he/she takes a 15 minutes break. During the rest break, part of his/her fatigue is alleviated and the remainder fatigue is carried over to the next workstation, where the worker performs a different job with different learning and fatigue rates. The worker continues moving from one job to the next till he/she comes back to the same 
workstation for the second, third, and $c^{\text {th }}$ time ( $c$ is the number of cycles). Each time the worker revisits the same workstation, he/she remembers some of his/her previous experience. Therefore, the entire time he/she was away from this station, on a rest break or working in other stations (learning break), causes him/her to forget. When the entire lot and its associated defects are processed, the performance measure is calculated from equation (7.3).

Figure 7.1 shows the possible scenarios to investigate in numerical examples. For example, one scenario considers increasing $(\uparrow) b$ and decreasing $(\downarrow) \lambda$, where a worker performs two different tasks $($ Flex $=2)$ in 15 cycles, which means the worker shifts from a slower to a faster learning and from a hard to an easy task. In total, $64(16 \times 2 \times 2)$ scenarios are created to examine specific research questions as follow. 


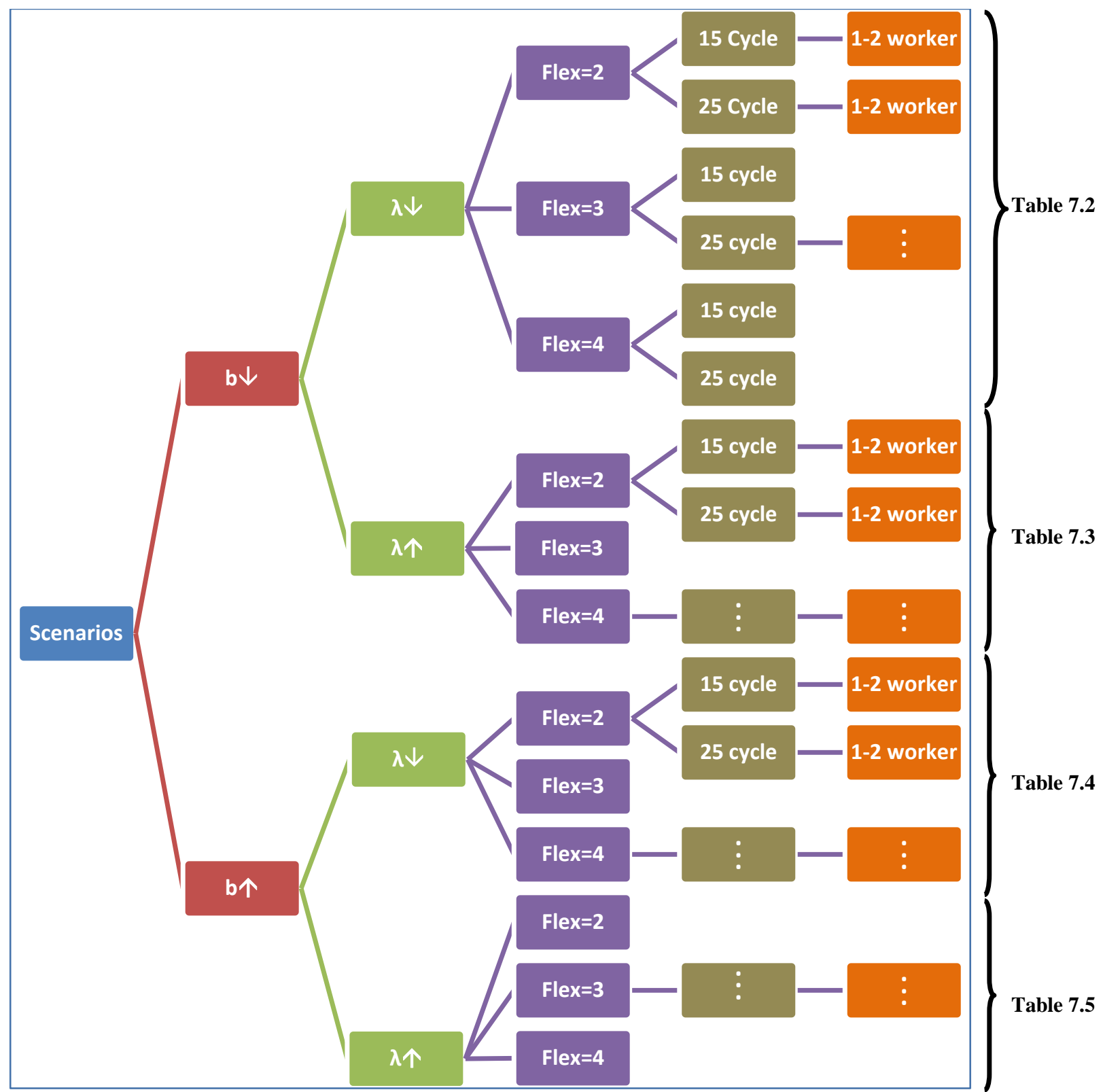

Figure 7.1. This figure demonstrates 64 different scenarios created to address the research questions. $b \downarrow$ $(\uparrow)$, indicates that learning is the fastest (slowest) at first and slowest (fastest) at the last workstation. $\lambda \downarrow$ ( ) indicates that the work is hardest (easiest) at the first and easiest (hardest) at the last workstation. The Flex number, identifies the number of workstations. Each scenario is repeated in 15 or 25 cycles with the assigned workers that can be 1,2, or 3. The Table \# indicates where results are presented. 


\subsubsection{Research questions}

In each scenario, we examine the following research questions:

\section{RQ-1: What is the optimal level of flexibility?}

This question examines the role of the level of flexibility in DRC systems. Since the flexibility level is equal to the number of skills a worker requires, this question answers one of the critical questions in DRC systems, that is, how many skills a worker should be trained for (Hottenstein \& Bowman, 1998)? In this Phase of the thesis, workstations represent flexibility since it is assumed that only one type of job can be performed at each workstation. For instance, Flex $=3$ means that a worker can (or is responsible to) perform three different tasks. The performance of the system is examined for Flex $=2$, Flex $=3$ and Flex $=4$. It should be noted that to stay within the context of the DRC system, the number of workers must always be less than or equal to the number of workstations or flexibility level.

\section{RQ-2: What is the optimal transfer policy?}

This question examines the "when rule" in DRC systems (when to transfer worker to another station?). The worker transfers to the next workstation after he/she produces and reworks the assigned batch and related defects in one cycle. Therefore, the smaller the batch, the faster is the transfer. The number of batches is equal to the number of cycles since each batch is processed in one cycle. The answer to this research question gives the number of cycles (or batches) that optimises the performance and sets the policy for production planning. 


\section{RQ-3: What is the best arrangement for workstations?}

This question examines one of the issues concerned in DRC systems that is, the effect of the workflow on the performance characteristics (Hottenstein \& Bowman, 1998). In this Phase, the workflow of the DRC system is affected by different learning and fatigue situations that a worker experiences at each station. The answer to this research question tells how the arrangement of workstations (high to low or low to high workloads) affects the workflow demonstrated and subsequently the performance of the system.

\subsection{Results}

\subsubsection{From fast to slow learning}

\subsubsection{From fast to slow learning and hard to easy work}

The results of solving these scenarios are presented in Table 7.2. With Flex=2, the system is run with 1 and 2 workers, for 15 and 25 work-cycles. With Flex $=3$ and Flex $=4$, the system can have up to 3 workers working in the same cycles. First, it is assumed that 9000 items are processed in 15 cycles. According to equation (7.6) each worker must process $\frac{9000}{15 \times m \times j}$ items and the defects per station per cycle, while $m$ is the number of workers and $j$ is the number of workstations. Therefore, for instance, if there are 3 workstations with 1 worker, the worker performs $\frac{9000}{15 \times 1 \times 3}=200$ items and the defects per station per cycle and he/she performs $200 \times 3 \times 15=9000$ in total (in 15 cycles). The results in Table 7.2 indicate that in this system, Flex=3 with 2 workers yields the best performance. Figure 7.2 demonstrates the performance of this system for Flex $=3$ ( 3 workstations) with the best performance obtained by 2 workers $(Z=1.6734)$ at the $14^{\text {th }}$ cycle. 
Table 7.2. Performance measure investigation when learning and fatigue indices decrease from the first to the last working station (Learning: $\mathrm{F}=\mathrm{Fast}, \mathrm{M}=$ Moderate, $\mathrm{S}=$ Slow; Workload: $\mathrm{H}=\mathrm{Hard}, \mathrm{M}=$ Moderate, $\mathrm{E}=$ Easy).

\begin{tabular}{|c|c|c|c|c|c|c|c|}
\hline Flex=2 & $\boldsymbol{b}$ & $\boldsymbol{\lambda}$ & & Cycle & Worker & $Z_{\max }$ & Cycle of $Z_{\max }$ \\
\hline WS1 & F: 0.515 & $\mathrm{H}: 0.19$ & & 15 & 1 & 1.62003 & 15 \\
\hline WS2 & S: 0.152 & $\mathrm{E}: 6.4 \times 10^{-3}$ & & & 2 & 1.6658 & 15 \\
\hline & & & & & - & - & - \\
\hline & & & & 25 & 1 & 1.6573 & 25 \\
\hline & & & & 2 & 1.6698 & 19 \\
\hline & & & & - & - & - \\
\hline Flex=3 & $\boldsymbol{b}$ & $\lambda$ & & Cycle & Worker & $Z_{\max }$ & Cycle of $Z_{\max }$ \\
\hline WS1 & F: 0.515 & $\mathrm{H}: 0.19$ & & 15 & 1 & 1.6573 & 15 \\
\hline WS2 & M: 0.322 & M: $9.6 \times 10^{-3}$ & & & 2 & 1.6734 & 14 \\
\hline WS3 & S: 0.152 & E: $6.4 \times 10^{-3}$ & & & 3 & 1.6721 & 10 \\
\hline & & & & 25 & 1 & 1.6679 & 23 \\
\hline & & & & & 2 & 1.6734 & 14 \\
\hline & & & & 3 & 1.6721 & 10 \\
\hline Flex=4 & $\boldsymbol{b}$ & $\lambda$ & & Cycle & Worker & $Z_{\max }$ & Cycle of $Z_{\max }$ \\
\hline WS1 & F: 0.515 & $\mathrm{H}: 0.19$ & & 15 & 1 & 1.6684 & 15 \\
\hline WS2 & M: 0.322 & M: $9.6 \times 10^{-3}$ & & & 2 & 1.6726 & 11 \\
\hline WS3 & M: 0.322 & M: $9.6 \times 10^{-3}$ & & & 3 & 1.6694 & 8 \\
\hline WS4 & S: 0.152 & E: $6.4 \times 10^{-3}$ & & 25 & 1 & 1.6705 & 18 \\
\hline & & & & & 2 & 1.6726 & 11 \\
\hline & & & & 3 & 1.6694 & 8 \\
\hline
\end{tabular}

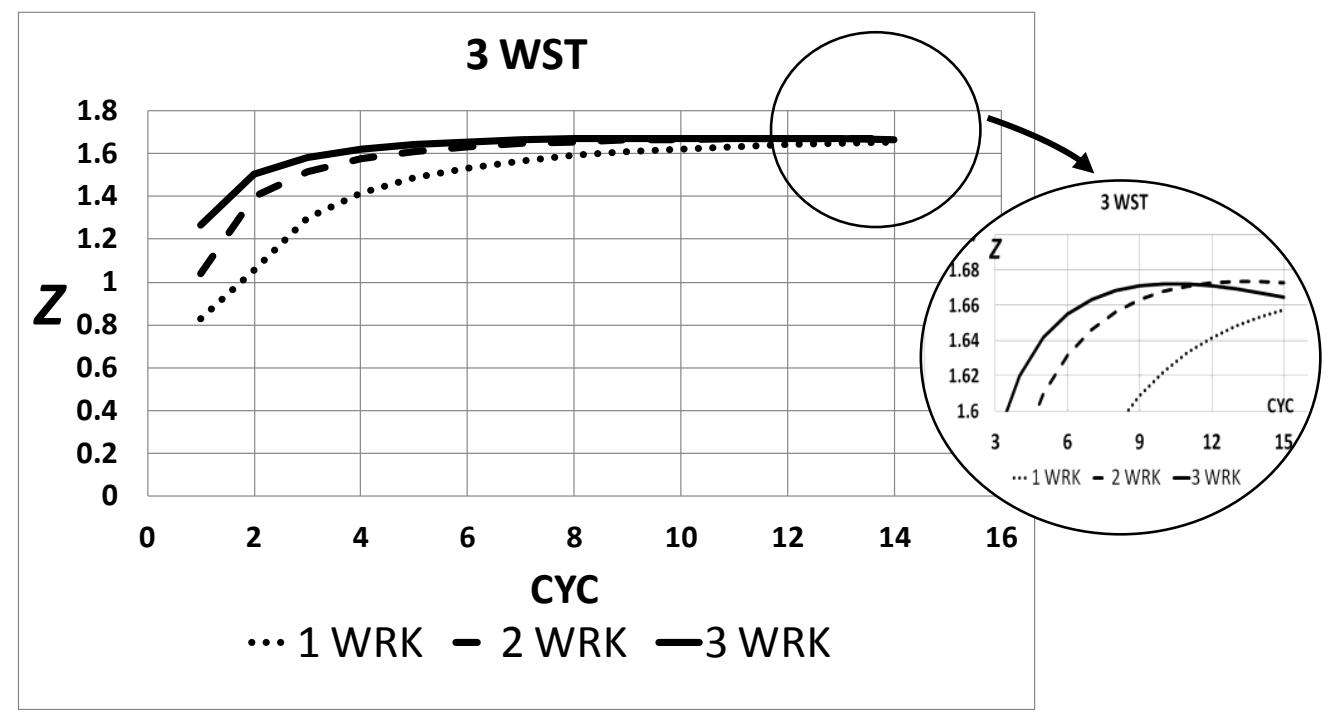

Figure 7.2. System performance $(Z)$ versus working cycles for 3 workstations and up to 3 workers with Flex $=3$ during 15 working cycles, learning and fatigue indices decrease from the first to the last working station. The inset provides a closer look of the performance of 2 workers declining after the $14^{\text {th }}$ and 3 workers declining after the $10^{\text {th }}$ cycle (WRK= Worker). 
The system performance $Z$ in Figure 7.2, consists of direct cost indicator $Z_{l}$ and indirect cost indicator $Z_{2}$ according to equation (7.3), which have been illustrated in Figure 7.4 and Figure 7.3 respectively. For conciseness and to avoid repetition, $Z_{1}$ and $Z_{2}$ will not be demonstrated for the remaining numerical examples of this Section.

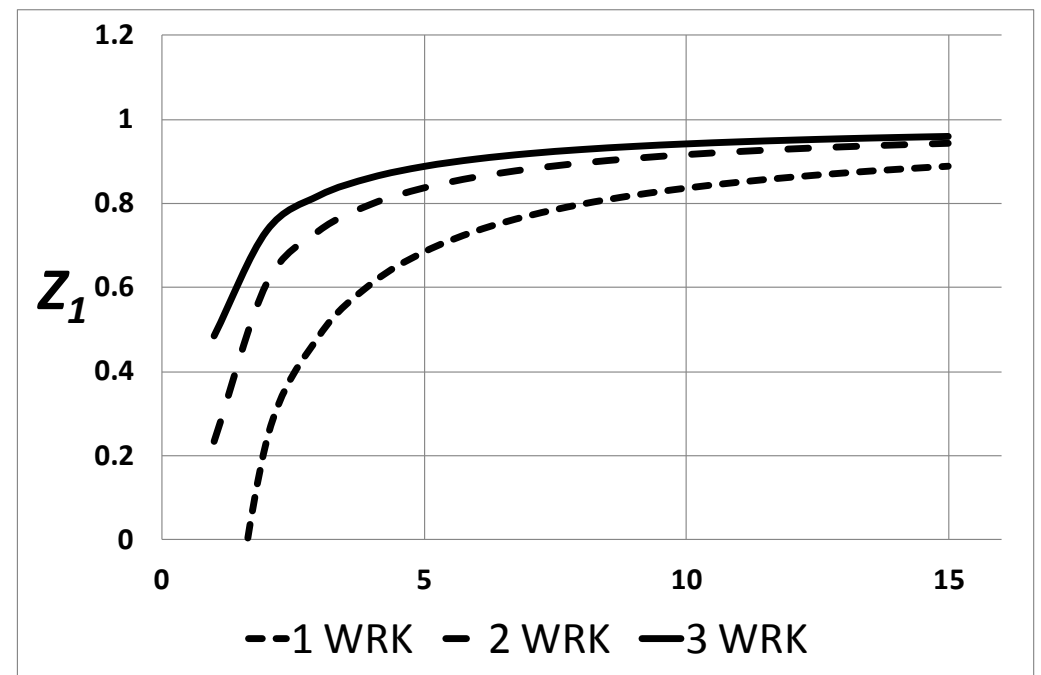

Figure 7.3. Performance indicator $Z_{1}$ increases asymptotically from zero over 15 working cycles for 3 workstations and up to 3 workers with Flex $=3$. Learning and fatigue indices decrease from the first to the last working station (WRK= Worker).

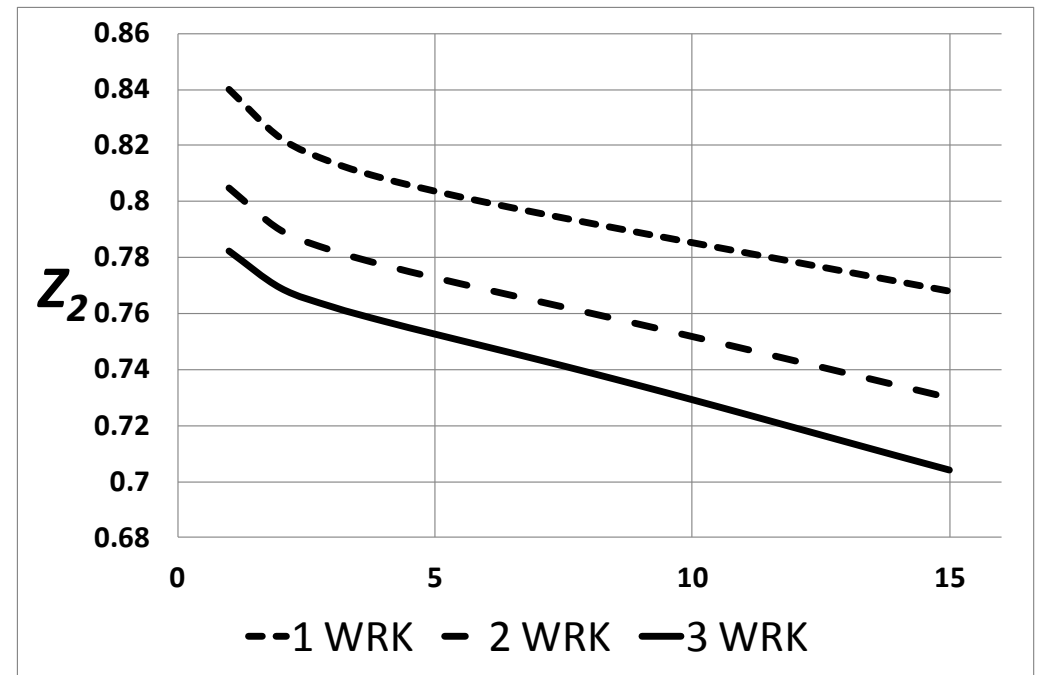

Figure 7.4. Performance indicator $Z_{2}$ decreases from one over 15 working cycles for 3 workstations and up to 3 workers with Flex $=3$. Learning and fatigue indices decrease from the first to the last working station (WRK= Worker). 


\subsubsection{Flexibility}

Returning to Table 7.2, the system performance of Flex $=4$ is compared with Flex=3. The workload is assumed to be similar to 7.3.1.1 (from fast to slow learning and hard to easy work), except that this time the two middle workstations have a moderate workload and learning. It is assumed that 9000 jobs are to be done by 1, 2, and 3 workers respectively in 15 cycles. Similar to 7.3.1.1, each worker must process $\frac{9000}{15 \times m \times j}$ items and the defects per station per cycle. The results are shown in Figure 7.5 where the best performance obtained by two workers at the $11^{\text {th }}$ cycle with $\mathrm{Z}=1.6726$. Comparing the result to Flex $=3$ with the best performance of $Z=1.6734$ at the $14^{\text {th }}$ cycle, it is concluded that the best performance of the system declines with increased flexibility and also occurs earlier, i.e., cycle 11 instead of 14 which answers RQ-1.

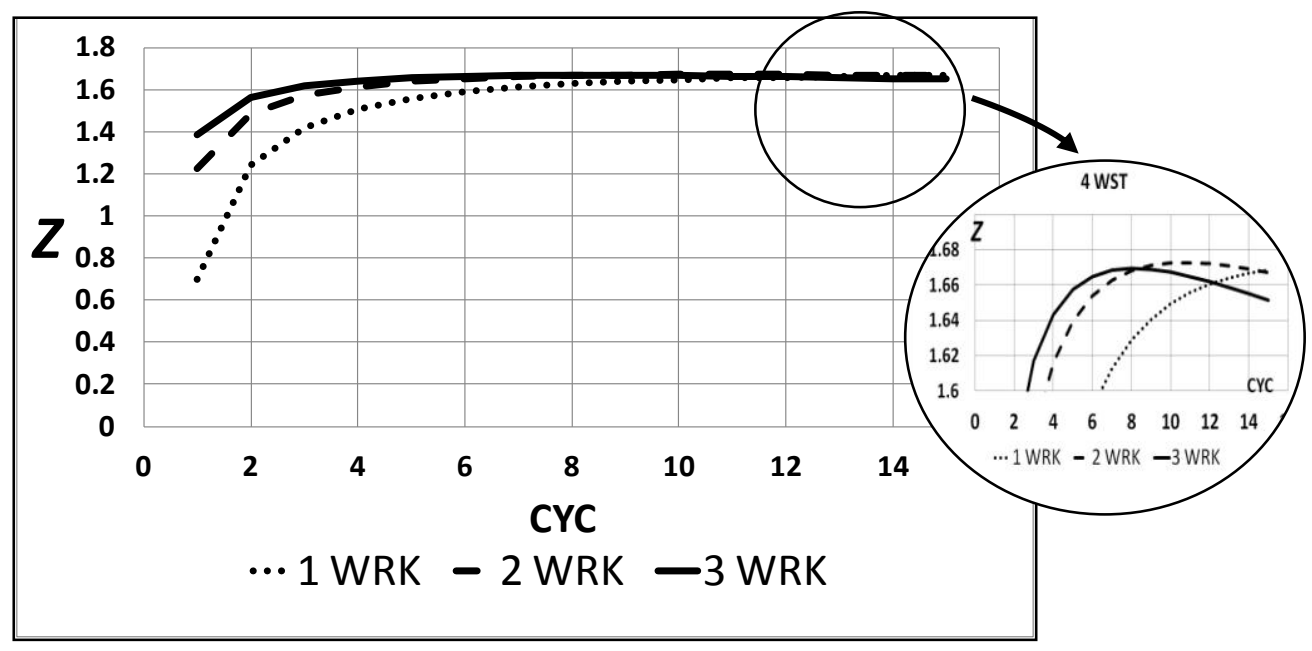

Figure 7.5. System performance $(Z)$ versus working cycles for 4 workstations and up to 3 workers with Flex $=4$ during 15 working cycles, learning and fatigue indices decrease from the first to the last working station. The inset provides a closer look of the performance of 2 workers declining after the $11^{\text {th }}$ and 3 workers declining after the $8^{\text {th }}$ cycle $(\mathrm{WRK}=$ Worker $)$. 


\subsubsection{Number of cycles}

To examine the effect of number of cycles, we return to Table 7.2 and investigate Flex=3 with 15 and 25 cycles. Only Flex $=3$ is considered since it corresponded to the best performance (in 0 )

with 15 cycles. With 25 cycles, each worker should process $\frac{9000}{25 \times m \times j}$ items and the defects per station per cycle. Figure 7.6 shows the performance of this system with the best performance obtained by two workers at the $14^{\text {th }}$ cycle with $Z=1.6734$. Comparing Figure 7.2 and Figure 7.6, it is observed that as the entire lot is processed in more cycles, the performance declines further from its optimal value at the $14^{\text {th }}$ cycle. Therefore, producing in 15 cycles is better than producing in 25 cycles, since in the latter case, more cycles are performed at off- optimal performance. This observation answers RQ-2.

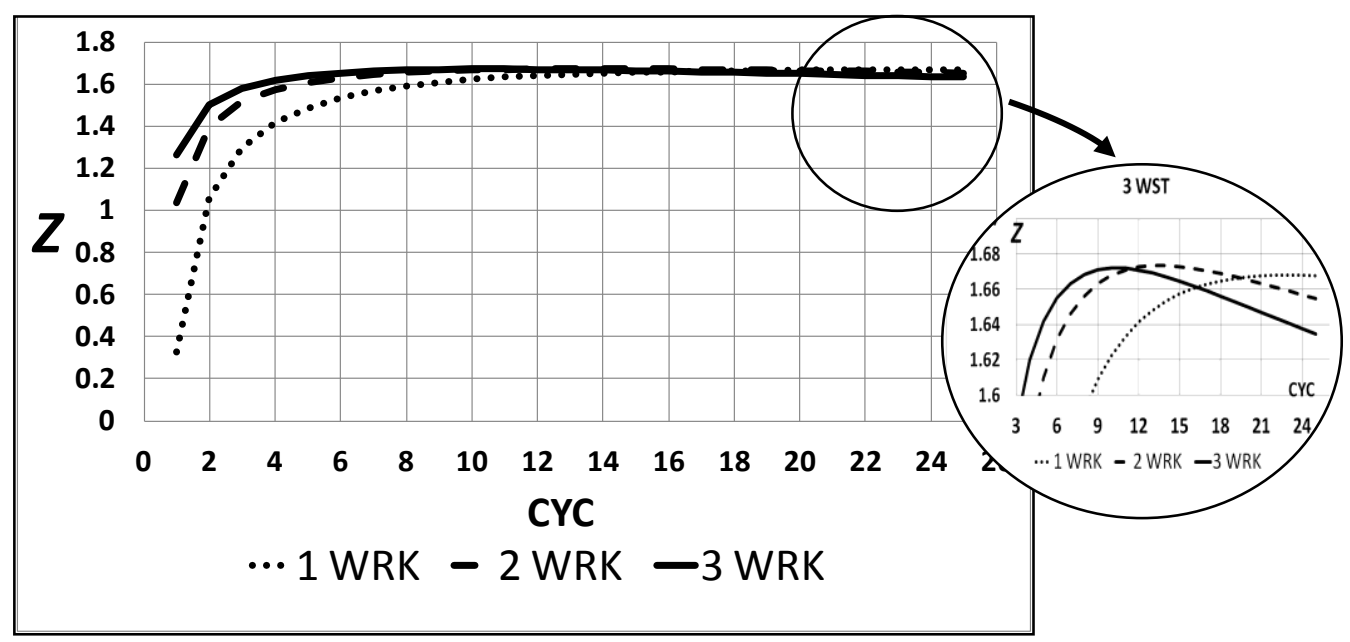

Figure 7.6. System performance $(Z)$ versus working cycles for 3 workstations and up to 3 workers with Flex $=3$ during 25 working cycles, learning and fatigue indices decrease from the first to the last working station. The inset provides a closer look of the performance of 2 workers declining after the $14^{\text {th }}$ and 3 workers declining after the $10^{\text {th }}$ cycle (WRK= Worker). 


\subsubsection{From fast to slow learning and easy to hard work}

With the same learning scheme (from fast to slow learning) an increasing workload is assumed, that is, the work is the easiest at the first, moderate at the second, and the hardest at the third workstation. The results of this case are represented in Table 7.4. Figure 7.7 demonstrates the performance of this system for Flex $=3$ ( 3 workstations), with the best performance obtained with 2 workers at the $13^{\text {th }}$ cycle as $Z=1.6744$. Results indicate that with a decreasing learning from the first station to the third, and the transfer of the worker from the easiest job to the hardest, the performance of the system improves and also obtained earlier in the process which also answers RQ-3.

Table 7.3. Performance measure investigation when learning index decreases and fatigue index increases from the first to the last working station (Learning: $\mathrm{F}=\mathrm{Fast}, \mathrm{M}=$ Moderate, $\mathrm{S}=\mathrm{Slow}$; Workload: $\mathrm{H}=\mathrm{Hard}$, $\mathrm{M}=$ Moderate, $\mathrm{E}=$ Easy).

\begin{tabular}{|c|l|l|l|c|c|c|c|}
\hline Flex=2 & $\boldsymbol{b}$ & \multicolumn{1}{|c|}{$\boldsymbol{\lambda}$} & & Cycle & Worker & $Z_{\max }$ & Cycle of $Z_{\max }$ \\
\hline WS1 & F: 0.515 & E: $6.4 \times 10^{-3}$ & & 15 & 1 & 1.6257 & 15 \\
\hline WS2 & S: 0.152 & H: 0.19 & & & 2 & 1.6681 & 15 \\
\hline & & & & & - & - & - \\
\hline & & & & 25 & 1 & 1.6606 & 25 \\
\hline & & & & 2 & 1.6711 & 18 \\
\hline & & & & - & - & - \\
\hline Flex=3 & $\boldsymbol{b}$ & $\lambda$ & & Cycle & Worker & $Z_{\max }$ & Cycle of $Z_{\max }$ \\
\hline WS1 & F: 0.515 & E: $6.4 \times 10^{-3}$ & & 15 & 1 & 1.6603 & 15 \\
\hline WS2 & M: 0.322 & M: $9.6 \times 10^{-3}$ & & & 2 & 1.6744 & 13 \\
\hline WS3 & S: 0.152 & H: 0.19 & & & 3 & 1.6726 & 10 \\
\hline & & & & 25 & 1 & 1.6696 & 22 \\
\hline & & & & & 2 & 1.6744 & 13 \\
\hline & & & & 3 & 1.6726 & 10 \\
\hline Flex=4 & $\boldsymbol{b}$ & $\lambda$ & & Cycle & Worker & $Z_{\max }$ & Cycle of $Z_{\max }$ \\
\hline WS1 & F: 0.515 & E: $6.4 \times 10^{-3}$ & & 15 & 1 & 1.6701 & 15 \\
\hline WS2 & M: 0.322 & M: $9.6 \times 10^{-3}$ & & & 2 & 1.6732 & 10 \\
\hline WS3 & M: 0.322 & M: $9.6 \times 10^{-3}$ & & & 3 & 1.6698 & 8 \\
\hline WS4 & S: 0.152 & H: 0.19 & & 25 & 1 & 1.6716 & 18 \\
\hline & & & & & 2 & 1.6732 & 10 \\
\hline & & & & & 3 & 1.6698 & 8 \\
\hline
\end{tabular}




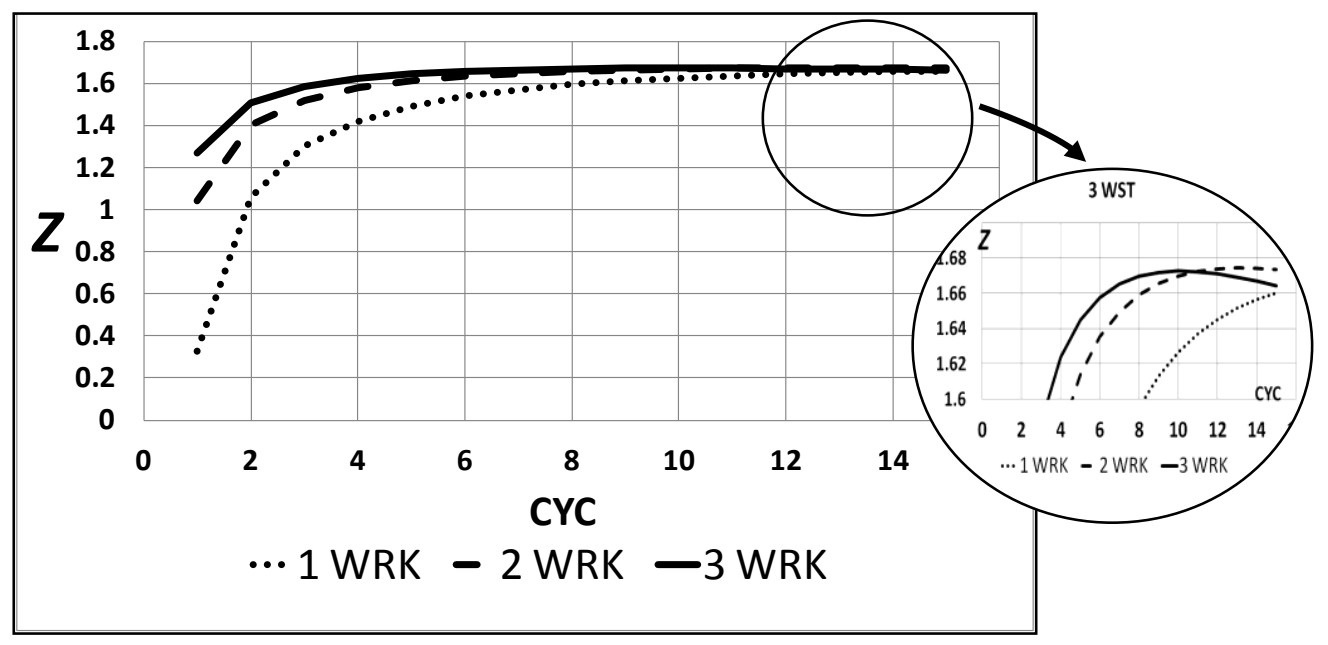

Figure 7.7. System performance $(Z)$ versus working cycles for 3 workstations and up to 3 workers with Flex $=3$ during 15 working cycles, learning index decreases and fatigue index increases from the first to the last working station. The inset provides a closer look of the performance of 2 workers declining after the $13^{\text {th }}$ and 3 workers declining after the $10^{\text {th }}$ cycle $($ WRK $=$ Worker $)$.

\subsubsection{From slow to fast learning}

\subsubsection{From slow to fast learning and hard to easy work}

The results of these scenarios are presented in Table 7.4. As before, with Flex=2, the system is run with 1 and 2 workers. With Flex=3, the system can have up to 3 workers working in the same cycles. The learning increases and the workload decreases from the first to the last station while the number of workstations depends on the Flex number. First, it is assumed that 9000 items are processed in 15 cycles. Again each worker must process $\frac{9000}{15 \times m \times j}$ items and the defects per station per cycle, while $m$ is the number of workers and $j$ is the number of workstations. According to Table 7.4 the best performance of this system obtained when Flex $=$ 3 with 2 workers $(Z=1.6744)$ at the $13^{\text {th }}$ cycle. 
Table 7.4. Performance measure investigation when learning increases and fatigue decreases from the first to the last working station (Learning: $\mathrm{F}=\mathrm{Fast}, \mathrm{M}=$ Moderate, $\mathrm{S}=\mathrm{Slow}$; Workload: $\mathrm{H}=\mathrm{Hard}$, $\mathrm{M}=$ Moderate, $\mathrm{E}=$ Easy).

\begin{tabular}{|c|c|c|c|c|c|c|}
\hline Flex $=2$ & $b$ & $\lambda$ & Cycle & Worker & $Z_{\max }$ & Cycle of $Z_{\max }$ \\
\hline WS1 & S: 0.152 & H: 0.19 & 15 & 1 & 1.6261 & 15 \\
\hline \multirow[t]{5}{*}{ WS2 } & F: 0.515 & E: $6.4 \times 10^{-3}$ & & 2 & 1.6681 & 15 \\
\hline & & & & - & - & - \\
\hline & & & 25 & 1 & 1.6614 & 25 \\
\hline & & & & 2 & 1.6711 & 18 \\
\hline & & & & - & & - \\
\hline Flex $=3$ & $b$ & $\lambda$ & Cycle & Worker & $Z_{\max }$ & Cycle of $Z_{\max }$ \\
\hline WS1 & S: 0.152 & H: 0.19 & 15 & 1 & 1.6607 & 15 \\
\hline WS2 & M: 0.322 & M: $9.6 \times 10^{-3}$ & & 2 & 1.6744 & 13 \\
\hline \multirow[t]{4}{*}{ WS3 } & F: 0.515 & E: $6.4 \times 10^{-3}$ & & 3 & 1.6725 & 10 \\
\hline & & & 25 & 1 & 1.6699 & 22 \\
\hline & & & & 2 & 1.6744 & 13 \\
\hline & & & & 3 & 1.6725 & 10 \\
\hline Flex $=4$ & $b$ & $\lambda$ & Cycle & Worker & $Z_{\max }$ & Cycle of $Z_{\max }$ \\
\hline WS1 & S: 0.152 & H: 0.19 & 15 & 1 & 1.6703 & 15 \\
\hline WS2 & M: 0.322 & M: $9.6 \times 10^{-3}$ & & 2 & 1.6732 & 10 \\
\hline WS3 & M: 0.322 & M: $9.6 \times 10^{-3}$ & & 3 & 1.6698 & 8 \\
\hline \multirow[t]{3}{*}{ WS4 } & F: 0.515 & E: $6.4 \times 10^{-3}$ & 25 & 1 & 1.6719 & 18 \\
\hline & & & & 2 & 1.6732 & 10 \\
\hline & & & & 3 & 1.6698 & 8 \\
\hline
\end{tabular}

\subsubsection{Flexibility}

Returning to Table 7.4, the system performance with Flex=4 is compared to Flex=3. The workload is assumed to be similar to 7.3.2.1 (from slow to fast learning and hard to easy work), except that this time the two middle workstations have moderate workloads and learning. It is assumed that 9000 jobs are to be done by 1,2, and 3 workers respectively in 15 cycles. Similar to 7.3.2.1, each worker must process $\frac{9000}{15 \times m \times j}$ items and the defects per station per cycle. The best performance obtained with two workers in the $10^{\text {th }}$ cycle with $Z=1.6732$. Comparing the results to those of section 7.3.2.1 with Flex $=3$ and best performance of $Z=1.6744$ at the $13^{\text {th }}$ cycle, it is observed that the best performance of the system declines with increased flexibility. The performance peak also occurs earlier, i.e., at cycle 10 instead of 14 which answers RQ-1. 


\subsubsection{Number of cycles}

To examine the effect of cycles, we return to Table 7.4 and investigate Flex=3 with 15 and 25 cycles. Again, only Flex=3 is considered since it corresponds to the best performance. With 25 cycles, each worker should process $\frac{9000}{25 \times m \times j}$ items and the defects per station per cycle. As per Table 7.4 , the best performance is obtained by two workers in the $13^{\text {th }}$ cycle with $Z=1.6744$. Comparing to 7.3.2.1 (from slow to fast learning and hard to easy work), as the entire lot is processed in more cycles, the best performance does not change since it reached its maximum value in the $13^{\text {th }}$ cycle and will not increase further. When producing a lot in more cycles however, the process performance is not optimal as it further decreases with increasing cycles (from cycle 13 to 25 ), which answers RQ-2.

\subsubsection{From slow to fast learning and easy to hard work}

With the same learning scheme (from slow to fast learning) an increasing workload is assumed, that is, the work is the easiest at the first, moderate at the second, and the hardest at the third workstation. The results of this case are summarised in Table 7.5 and show the best performance is obtained with Flex $=3$ and 2 workers in cycle 14 where $Z=1.6735$. The results indicate that as the learning becomes faster as a worker moves from one station to the next and the worker transfers from the easiest job to the hardest, the performance of the system declines and is also obtained later in the process which answers RQ-3 (compare the corresponding result in Table 7.4 with $\mathrm{Z}=1.6744$ at cycle 13 and the one in Table 7.5 with $\mathrm{Z}=1.6735$ at cycle 14 ). 
Table 7.5. Performance measure investigation when learning and fatigue indices increase from the first to the last working station (Learning: $\mathrm{F}=\mathrm{Fast}, \mathrm{M}=$ Moderate, $\mathrm{S}=$ Slow; Workload: $\mathrm{H}=\mathrm{Hard}, \mathrm{M}=$ Moderate, $\mathrm{E}=$ Easy).

\begin{tabular}{|c|c|c|c|c|c|c|c|}
\hline Flex=2 & $\boldsymbol{b}$ & $\boldsymbol{\lambda}$ & & Cycle & Worker & $Z_{\max }$ & Cycle of $Z_{\max }$ \\
\hline WS1 & S: 0.152 & $\mathrm{E}: 6.4 \times 10^{-3}$ & & 15 & 1 & 1.6205 & 15 \\
\hline WS2 & F: 0.515 & $\mathrm{H}: 0.19$ & & & 2 & 1.6658 & 15 \\
\hline & & & & & - & & \\
\hline & & & & 25 & 1 & 1.6581 & 25 \\
\hline & & & & & 2 & 1.6698 & 19 \\
\hline & & & & & - & & - \\
\hline Flex=3 & $\boldsymbol{b}$ & $\lambda$ & & Cycle & Worker & $Z_{\max }$ & Cycle of $Z_{\max }$ \\
\hline WS1 & S: 0.152 & E: $6.4 \times 10^{-3}$ & & 15 & 1 & 1.6579 & 15 \\
\hline WS2 & M: 0.322 & M: $9.6 \times 10^{-3}$ & & & 2 & 1.6735 & 14 \\
\hline WS3 & F: 0.515 & H: 0.19 & & & 3 & 1.6720 & 10 \\
\hline & & & & 25 & 1 & 1.6685 & 23 \\
\hline & & & & & 2 & 1.6735 & 14 \\
\hline & & & & & 3 & 1.6720 & 10 \\
\hline Flex=4 & $\boldsymbol{b}$ & $\lambda$ & & Cycle & Worker & $Z_{\max }$ & Cycle of $Z_{\max }$ \\
\hline WS1 & S: 0.152 & $\mathrm{E}: 6.4 \times 10^{-3}$ & & 15 & 1 & 1.6688 & 15 \\
\hline WS2 & M: 0.322 & M: $9.6 \times 10^{-3}$ & & & 2 & 1.6727 & 11 \\
\hline WS3 & M: 0.322 & M: $9.6 \times 10^{-3}$ & & & 3 & 1.6694 & 8 \\
\hline WS4 & F: 0.515 & H: 0.19 & & 25 & 1 & 1.6708 & 18 \\
\hline & & & & & 2 & 1.6727 & 11 \\
\hline & & & & & 3 & 1.6694 & 8 \\
\hline
\end{tabular}

\subsection{Discussion and conclusion}

Not considering human restrictions and abilities will result in unrealistic production planning that could underestimate or overestimate production costs. In this Phase, a more comprehensive model for DRC systems considering human aspects was developed. This Phase is a new approach that locates the human in the center of industrial planning. Considering that production lines are still heavily dependent on the human performance, this thesis contributes to the scarce literature investigating the effect and role of human factors on the process quality and output.

The models that were developed in the previous Phases for human behaviour, i.e., the Learning Forgetting Fatigue Recovery Model (LFFRM of Phase 1), the Quality Learning Forgetting Curve 
(QLFC of Phase 2), and also a model for human error making with learning and fatigue (of Phase 3) were used to develop and analyze the production planning for DRC systems. The developed model simulates a production process in which flexible workers transfer between different workstations and each station provokes certain types of learning and fatigue. When performing a work, the level of learning and fatigue contribute to the error making of worker who can generate defective products. However, defective items could also be generated as a result of machine malfunction. When a production lot arrives at the first station of a system, it is processed in cycles with the associated defects. The performance of the system was studied with a twofold measure function consisting direct (time) and indirect (quality) costs.

Also the effects of learning and fatigue rates, number of cycles, and the flexibility of workers were investigated by addressing three research questions. To answer the first research question (RQ-1), the desired level of flexibility was studied. The results showed that irrespective of the configuration of learning and fatigue, number of workers, or number of cycles, Flex $=3$ always yielded better results than Flex $=2$ or Flex $=4$. This corroborates the finding of Kim and Nembhard (2010), Kher et al. (1999), and Jaber et al. (2003) that the best level of flexibility in DRC systems is 2 or 3 .

To answer the second research question (RQ-2), the transfer policy of workers was examined. The results showed that irrespective of the pattern of learning and fatigue, flexibility level, or the number of workers, there is an optimum number of cycles beyond which, the performance of the system deteriorates. This result is compatible with the works of Jaber and Guiffrida (2004) and Jaber and Guiffrida (2008), where they observed that the quality learning curve has an optimum performance level when the rework learning rate is less than or equal to the production learning 
rate. This result also determines the transfer policy; i.e. the entire lot must be processed in a number of cycles that optimises the performance. If a lot is processed in more cycles than the optimal number, then the frequency of transfer of a worker increases which is lowering the performance. This finding is corroborated by Kher et al. (1999) who concluded that the effect of forgetting as a result of increasing flexibility and transfers can be encountered by processing larger batch sizes.

The work performed at each workstation and the configuration of workstations could have various effects on the learning behaviour, with cognitive aspects, and fatigue behaviour, with motor aspects and result to various levels of quality of products. The results show that performing the tasks sequentially from the hardest to the easiest, adjacent to low to high learning rates, results to a process of the highest performance. The second best performance obtained in a system with workstations associated with high to low learning and low to high fatigue rates. The third research question reveals that a system with the opposite arrangement of learning and fatigue rates will yield the best performance.

The managerial implications of this Phase of the thesis indicate that increasing the number of workers, working cycles, or the skills flexibility does not necessarily result in a better performance of the system. Other hidden factors such as quality issues, fatigue, and forgetting of workers are involved that have negative effects and deteriorate the performance in cases of excessive flexibility or transfer rate of workers. The results also arrest the attention of managers towards the workflow and its effect on the process outcomes. The workflow was addressed by configuration of workstations in this Phase of the thesis and it has shown to affect the optimality conditions of the working system. 


\section{Contributions and Future Directions}

DRC system models that considered human aspects are scarce. Aspects such as learning, forgetting, fatigue, and recovery that impact performance of DRC systems and the quality of the final product have been studied either independently or in combination of two (e.g., learning and forgetting). There is no study in the literature that considers the combined effects of these factors. The aim of this research was to fill this gap by studying how these human aspects interact and how they affect the system performance and throughput. This thesis revised traditional scheduling and production planning methods that ignore human aspects. New mathematical models were developed in four Phases, of which the last is a comprehensive model, which integrates learning, forgetting, fatigue, and recovery with product and process quality in a DRC setting. Worker flexibility and transfer policies, which are fundamentals of DRC systems, were also studied in the specified context. This has become growingly popular among manufacturing firms that wish to be responsive, flexible, and efficient, where fewer workers are needed to perform the same number of tasks.

\subsection{Contributions}

In Phase 1 of this thesis, fatigue and recovery were incorporated into an existing learning and forgetting model to study how the four human aspects interplay. The resultant model was used to study human behavior in a workplace where mentioned aspects may be present and to investigate the effects of their interactions on the performance of a human-centred production system. The results indicated that learning and recovery and forgetting and fatigue have opposite effects on the system performance, which can be optimized by a trade-off among the human aspects. The 
results of Phase 1 suggested that workers' capabilities and limitations are critical issues that must be considered when designing production systems that are labour intensive.

In Phase 2, lot sizing and batching policies were investigated in the presence of learning and forgetting and product and process quality where the interruption of the production process is allowed to restore its quality. An optimization method was developed for determining the optimal policies. This Phase suggested how product and process quality can be improved in a human centred production system and how the standard time can be improved (reduced) as a result. The results of this Phase were later used in developing the model of Phase 4.

In Phase 3, the confluence of learning-forgetting and fatigue-recovery and their effects on the reliability of a worker performing a manual task was investigated. A Human Reliability Analysis model was developed to measure human error when performing a repetitive task over a period of time. This Phase captured how human reliability changes over time in a production process and optimized the production throughput with regard to human reliability.

In Phase 4, a DRC system model was developed in which, workers contribute to the performance of the system and product quality. Using the models developed in Phases 1 to 3; which are the Learning Forgetting Fatigue Recovery Model (LFFRM) in Phase 1, Quality Learning Forgetting Curve (QLFC) model in Phase 2, and Human Reliability Analysis model in Phase 3, the production planning and flexibility policies in a DRC system were investigated through a twofold cost function. Phase 4, which completes this thesis, presented an inclusive model of a DRC system which is capable of relating system characteristics (flexibility and transfer policies) to human behavior (learning-forgetting, fatigue-recovery, and error making). This model provided some insights into how managers can balance between system performance and 
throughput and workers welfare. The results indicated that in order to obtain reasonable and applicable policies for production planning and job scheduling, it is suggested that human characteristics be considered when designing DRC systems.

\subsection{Limitations}

The limitations of the research presented in this thesis are:

\subsubsection{Benchmarking}

Modelling and analyzing human behavior is a complex subject. Although there are many models that represent the learning and forgetting process, the fatigue and recovery process, and error generation mechanisms, there is no consensus among researchers to which one to use in which setting and under what conditions, and many models either have not been validated empirically or have been tested in experimental settings that resemble a manufacturing environment. For example, there are at least 10 groups of univariate and multivariate learning curve models (Badiru, 1992), 10 forgetting models (Nembhard \& Osothsilp, 2001; Sikström \& Jaber, 2002, 2012), 8 groups of fatigue (Dawson et al., 2011), and 4 groups of recovery models (El ahrache \& Imbeau, 2009) to be used in various areas. Furthermore, some models may have been developed for specific applications, such as Rose's models (1992) for fatigue and recovery of construction workers. Also, in some situations, it is not possible to distinguish some aspects of human behaviour such as fatigue or boredom or to separate cognitive from motor learning. These limitations make the benchmarking of the results restrictive and conservative since it is not possible to track the behavior of each outcome to the related individual sources. 


\subsubsection{Data availability}

Analytical models need empirical data to be validated; however, such is scarce, especially, when it involves the characteristics of workers. Also, as the literature shows, human behaviour in the workplace has been measured by subjective methods. Translating such data to quantitative ones that can be used to validate mathematical models may be questionable. Of course, having available data will give a modeller the opportunity to test his/her model against data, measure deviation and revisit the model for tuning and fine-tuning until a model produces the output that reasonably conforms to the collected data. Although the results of this thesis are useful for setting strategies for performance measurement, management, and improvement, they cannot be generalised in the absence of empirical validation.

\subsubsection{Assumptions}

Another limitation is in some of the assumptions made in this thesis. For example, in this thesis it was assumed that the fatigue level has a maximum of one and a minimum of zero, and that the fatigue function is exponential. It may be possible that fatigue has a minimum residual value and a maximum threshold value and the form of the fatigue accumulation function may be of different form, say S curve. There was no model in the literature that quantifies general fatigue and unfortunately, the behaviour of the fatigue accumulation found in the literature was descriptive. These assumptions raise other fundamental questions that if answered, may somewhat affect the results of this thesis. For example:

- How much learning can a worker really achieve during a work shift?

- How much fatigue a worker is allowed to/can accumulate in a work shift? 
Answering the above questions probably will change outcomes of the developed models and the managerial insights and implications.

\subsubsection{Interactions}

A human being is a complicated system. It consists of motor and mental functions that interact with one another. It is not yet known how they interact and to the author's knowledge there is no mathematical model that captures that interaction. This raises several interesting research questions, which are:

- What type of a job generates what kind of fatigue?

- How do physical and psychosocial aspects of human behavior interact with each other, and how one affects the other?

- How does the type of fatigue affect the rate and duration of recovery?

- Does fatigue limit the learning process? Does recovery, improve it?

- What is the effect of age, gender, or skill level on learning and fatigue behaviour?

\subsection{Future directions}

The models provided in this thesis could be improved by removing some of the aforementioned limitations. Benchmarking the analytical models with precise data, subtle knowledge of human cognitive and motor functions and their interactions, as well as reliable assumptions, improve the modeling and yield more realistic results.

A future work of Phase 1 could consider a process with dynamic scheduling for break times and their frequency. The LFFRM was studied for processing of a fixed number of batches and 
breaks. A dynamic program could be developed to have enough flexibility for scheduling an optimized process for varying number and frequency of batches and break times.

Phase 2 assumed that defective items are reworked and there is no double defected (scrap) item. Also it was assumed that restoration times are negligible in comparison to the cycle time. More insights into this problem are needed to determine the optimal restoration policy to be used regarding the above limitations.

In Phase 3 the effect of rushing on a job or meeting a due-date (Myszewski, 2010), was not considered. Also, worker's learning ability was assumed not to be affected by fatigue. Future work could extend this Phase by removing these limitations.

In Phase 4 it was assumed that the recovery rate remains at the medium level throughout the entire work and the rework process has the same learning rates as the production process. A future work could address these limitations and also consider the effect of heterogeneous or homogenous workforce, job similarity, upfront training, and behavioral interaction effects.

The future work of this research in general could extend upon the following areas:

- Considering a heterogeneous workforce with a wide range of learning, forgetting, fatigue, recovery, and therefore error making possibilities,

- Estimating the effect of worker turnover, new hiring, upfront training, and organizational learning in the production planning, and

- Using more complex models such as multivariate models for learning and forgetting that consider the interactions of several factors in estimating the improvement in learning or cost reduction of the process. 


\section{Summary and Conclusions}

DRC systems include two types of resources: workers and machines (jobs). When workers alternate between different jobs the productivity of the system is affected by their learningforgetting, fatigue-recovery, and error making behaviour. Machines also could influence the production process by malfunctioning.

This thesis provided a model for DRC systems that take these characteristics into consideration and merged them into a single and comprehensive model. It relates worker characteristics of learning and forgetting, fatigue and recovery, and error generation, and malfunctioning behaviour of machines. The model was created in four Phases. In the first Phase, a learningforgetting model was modified by accounting for fatigue and recovery to develop the Learning Forgetting Fatigue Recovery Model (LFFRM) for a worker. In the second Phase, it assumed that the quality of a production process may deteriorate, thus generating defective items that require rework. This Phase, illustrated a system in which the products are not $100 \%$ quality accepted and need rework. In Phase 3, a human error model was developed and integrated into the LFFRM. Lastly, in Phase 4, a comprehensive model was developed that integrated the models developed in Phases 1 to 3 into a DRC system where the quality of the production process is imperfect. The developed model was investigated for production planning with different transfer and flexibility policies. This thesis represented a DRC model that resembles a real setting to some extent, and provided insights for improving the performance and throughput of DRC systems while considering workers' welfare. The results of this research are also applicable to manufacturing and service environments that are labor intensive. Aircraft manufacturing and hospitals are 
examples of such environments where the learning, forgetting, fatigue, recovery, and error making of workers may have significant effects on the quality of products and services delivered. 


\section{Appendix I}

To derive the right side of equation (4.14), we have $0.5\left(u_{i}+x_{i}\right) \geq\left(u_{i}+x_{i}\right)^{1+f_{i} / b}\left(u_{i}+x_{i}+S_{i}\right)^{-f_{i} / b}$, from which we have

$$
\begin{aligned}
& S_{i} \leq\left\{\frac{0.5\left(u_{i}+x_{i}\right)}{\left(u_{i}+x_{i}\right)^{1+f_{i} / b}}\right\}^{-b / f_{i}}-u_{i}-x_{i} \\
& \Rightarrow \quad\left[\frac{1-b}{T_{1}} \tau_{i}+\left(u_{i}+x_{i}\right)^{1-b}\right]^{1 /(1-b)}-u_{i}-x_{i} \leq\left\{\frac{0.5\left(u_{i}+x_{i}\right)}{\left(u_{i}+x_{i}\right)^{1+f_{i} / b}}\right\}^{-b / f_{i}}-u_{i}-x_{i} \\
& \Rightarrow \quad\left[\frac{1-b}{T_{1}} \tau_{i}+\left(u_{i}+x_{i}\right)^{1-b}\right]^{1 /(1-b)} \leq\left\{\frac{0.5\left(u_{i}+x_{i}\right)}{\left(u_{i}+x_{i}\right)^{1+f_{i} / b}}\right\}^{-b / f_{i}} \\
& \Rightarrow \quad \frac{1-b}{T_{1}} \tau_{i}+\left(u_{i}+x_{i}\right)^{1-b} \leq\left\{\frac{0.5\left(u_{i}+x_{i}\right)}{\left(u_{i}+x_{i}\right)^{1+f_{i} / b}}\right\}^{-b(1-b) / f_{i}} \\
& \Rightarrow \quad \tau_{i} \leq \frac{T_{i}}{1-b}\left\{\left[\frac{0.5\left(u_{i}+x_{i}\right)}{\left(u_{i}+x_{i}\right)^{1+f_{i} / b}}\right]^{-b(1-b) / f_{i}}-\left(u_{i}+x_{i}\right)^{1-b}\right\}
\end{aligned}
$$




\section{References}

Adler, G. L., \& Nanda, R. (1974). Effects of learning on optimal size determination- multiple product case. AIIE Trans, 6(1), 21-27.

Åhsberg, E. (1998). Perceived fatigue related to work. PhD, University of Stockholm, Sweden.

Åhsberg, E. (2000). Dimensions of fatigue in different working populations. Scandinavian Journal of Psychology, 41(3), 231-241.

Anderlohr, G. (1969). What production breaks cost. Industrial Engineering, 20(September), 34-36.

Anderson, M. C. (2003). Rethinking interference theory: Executive control and the mechanisms of forgetting. Journal of Memory and Language, 49(4), 415-445.

Anzanello, M. J., \& Fogliatto, F. S. (2011). Learning curve models and applications: Literature review and research directions. International Journal of Industrial Ergonomics, 41(5), 573-583.

Argote, L. (2013). Organizational learning: Creating, retaining and transferring knowledge: Springer.

Argote, L., \& Epple, D. (1990). Learning curves in manufacturing. Science, 247(4945), 920-924.

Aryanezhad, M., Kheirkhah, A., Deljoo, V., \& Mirzapour Al-e-hashem, S. (2009). Designing safe job rotation schedules based upon workers' skills. The International Journal of Advanced Manufacturing Technology, 41(1-2), 193-199.

Asfaw, A., Pana-Cryan, R., \& Rosa, R. (2011). The business cycle and the incidence of workplace injuries: Evidence from the U.S.A. Journal of Safety Research, 42(1), 1-8.

Azizi, N., \& Liang, M. (2013). An integrated approach to worker assignment, workforce flexibility acquisition, and task rotation. Journal of the Operational Research Society, 64(2), 260-275.

Azizi, N., Zolfaghari, S., \& Liang, M. (2010). Modeling job rotation in manufacturing systems: The study of employee's boredom and skill variations. International Journal of Production Economics, $123(1), 69-85$.

Badiru, A. B. (1992). Computational survey of univariate and multivariate learning curve models. IEEE Transactions on Engineering Management, 39(2), 176-188.

Badiru, A. B. (1995). Multivariate analysis of the effect of learning and forgetting on product quality. International Journal of Production Research, 33(3), 777-794.

Bailey, C. D. (1989). Forgetting and the Learning Curve: A Laboratory Study. Management Science, $35(3), 340-352$.

Bapna, R., Langer, N., Mehra, A., Gopal, R., \& Gupta, A. (2013). Human Capital Investments and Employee Performance: An Analysis of IT Services Industry. Management Science, 59(3), 641658. 
Barker, L. M., \& Nussbaum, M. A. (2011). Fatigue, performance and the work environment: a survey of registered nurses. Journal of advanced nursing, 67(6), 1370-1382.

Bechtold, S. E., \& Sumners, D. W. L. (1988). Note-Optimal Work-Rest Scheduling with Exponential Work-Rate Decay. Management Science, 34(4), 547-552.

Bello, G., \& Colombari, V. (1980). Empirical technique to estimate operator's errors (TESEO). Reliability Engineering, 1(3), 3-24.

Benkard, C. (2000). Learning and forgetting: the dynamics of aircraft production. American Economic Review, 90(4), 1034-1054.

Bevilacqua, M., Ciarapica, F. E., \& Mazzuto, G. (2012). Analysis of injury events with fuzzy cognitive maps. Journal of Loss Prevention in the Process Industries, 25(4), 677-685.

Bjork, E. L., \& Bjork, R. A. (2011). Making things hard on yourself, but in a good way: Creating desirable difficulties to enhance learning. In M. A. Gernsbacher, R. W. Pew, L. M. Hough \& J. R. Pomerantz (Eds.), Psychology and the real world: Essays illustrating fundamental contributions to society (pp. 56-64). New York: Worth Publishers.

Bokhorst, J. A., \& Gaalman, G. J. (2009). Cross-training workers in Dual Resource Constrained systems with heterogeneous processing times. International Journal of Production Research, 47(22), 6333-6356.

Boring, R. L., \& Blackman, H. S. (2007). The origins of the SPAR-H method's performance shaping factor multipliers. Paper presented at the Human Factors and Power Plants and HPRCT 13th Annual Meeting, 2007 IEEE 8th.

Burke, R. J., \& Fiksenbaum, L. (2008). Work Hours, Work Intensity, and Work Addiction: Costs and Benefits\$. The long work hours culture: causes, consequences and choices, 2, 1.

Burr, W., \& Pearne, N. (2013). Learning curve theory and innovation. Circuit World, 39(4), 169-173.

Cacciabue, P. C. (1998). Modelling and simulation of human behaviour for safety analysis and control of complex systems. Safety Science, 28(2), 97-110.

Carlson, J. G., \& Row, R. J. (1976). How much does forgetting cost? Industrial Engineering, 8(9), 40-47.

Carnahan, B. J., Redfern, M. S., \& Norman, B. (2000). Designing safe job rotation schedules using optimization and heuristic search. Ergonomics, 43(4), 543-560.

Carr, G. W. (1946). Peacetime cost estimating requires new learning curves. Aviation, 45(Journal Article).

Chaffin, D. B., Andersson, G. B. I., \& Martin, B. J. (2006). Occupational Biomechanics (Vol. 4). New York, NY: John Wiley \& Sons.

Chand, S. (1989). Lot sizes and setup frequency with learning in setups and process quality. European Journal of Operational Research, 42(2), 190-202. 
Chatzimichali, A., \& Tourassis, V. D. (2007). An empirical investigation of learning curve laws in the tile manufacturing industry. Paper presented at the International Conference on Industrial Engineering and Engineering Management, 2007 IEEE.

Chaudhuri, A., \& Behan, P. O. (2004). Fatigue in neurological disorders. The Lancet, 363(9413), 978988.

Cherrington, J., Lippert, S., \& Towill, D. R. (1987). Effect of prior experience on learning curve parameters. International Journal of Production Research, 25(3), 399-411.

Choo, A. S., Linderman, K. W., \& Schroeder, R. G. (2007). Method and psychological effects on learning behaviors and knowledge creation in quality improvement projects. Management Science, 53(3), 437-450.

Cochran, E. B. (1973). Dynamics of work standards. Manufacturing Engineering and Management, 70(28-31).

Crawford, J. (1944). Statistical accounting procedures in aircraft production. Aero Digest, 44, 78.

Dar-El, E. M. (2000). Human Learning: From Learning Curves to Learning Organizations, Part 36. Netherlands: Kluwer Academic Publishers.

Dar-El, E. M., Ayas, K., \& Gilad, I. (1995). A dual-phase model for the individual learning process in industrial tasks. IIE Transactions, 27(3), 265-271.

Dawson, D., Ian Noy, Y., Härmä, M., Åkerstedt, T., \& Belenky, G. (2011). Modelling fatigue and the use of fatigue models in work settings. Accident Analysis \& Prevention, 43(2), 549-564.

De Vries, J., Michielsen, H., \& Van Heck, G. (2003). Assessment of fatigue among working people: a comparison of six questionnaires. Occupational and Environmental Medicine, 60(suppl 1), i10i15.

Dean, G. E., Scott, L. D., \& Rogers, A. E. (2006). Infants at risk: when nurse fatigue jeopardizes quality care. Advances in Neonatal Care, 6(3), 120-126.

Dhillon, B. S. (2007). Mathematical Models for Predicting Human Reliability and Error in Transportation Systems Human reliability and error in transportation systems (pp. 145-164): Springer.

Dhillon, B. S. (2009). Human Reliability, Error, and Human Factors in Engineering Maintenance: with reference to Aviation and Power Generation: CRC Press, Taylor and Francis Group.

Di Pasquale, V., Iannone, R., Miranda, S., \& Riemma, S. (2013). An Overview of Human Reliability Analysis Techniques in Manufacturing Operations. In M. M. Schiraldi (Ed.), Operations Management (pp. 221-240): InTech, Chapters.

Dinges, D. F. (1995). An overview of sleepiness and accidents. Journal of sleep research, 4(S2), 4-14.

Dionisio, A. N. (2010). Air traffic controller fatigue and human error. PhD, Embry-Riddle Aeronautical University. 
Dodé, P. (2011). The Integration of Human Factors into Discrete Event Simulation and Technology Acceptance in Engineering Design. Master of Applied Science (MASc), Ryerson University, Toronto, Canada.

Dutton, J. M., \& Thomas, A. (1984). Treating progress functions as a managerial opportunity. Academy of Management Review, 9(2), 235-247.

El ahrache, K., \& Imbeau, D. (2009). Comparison of rest allowance models for static muscular work. International Journal of Industrial Ergonomics, 39(1), 73-80.

El ahrache, K., Imbeau, D., \& Farbos, B. (2006). Percentile values for determining maximum endurance times for static muscular work. International Journal of Industrial Ergonomics, 36(2), 99-108.

El Saadany, A. (2009). Inventory management in reverse logistics with imperfect production, learning, lost sales, subassemblies, and price/quality considerations. Doctor of Philosophy (PhD), Ryerson University, Toronto, Canada.

Elmaghraby, S. E. (1990). Economic manufacturing quantities under conditions of learning and forgetting (EMQ/LaF). Production Planning \& Control, 1(4), 196-208.

ElMaraghy, W. H., Nada, O. N., \& ElMaraghy, H. A. (2008). Quality prediction for reconfigurable manufacturing systems via human error modelling. International Journal of Computer Integrated Manufacturing, 21(5), 584-598.

Escorpizo, R. (2008). Understanding work productivity and its application to work-related musculoskeletal disorders. International Journal of Industrial Ergonomics, 38(3), 291-297.

Feldman, L. S., Cao, J., Andalib, A., Fraser, S., \& Fried, G. M. (2009). A method to characterize the learning curve for performance of a fundamental laparoscopic simulator task: defining "learning plateau" and "learning rate". Surgery, 146(2), 381-386.

Ferioli, F., Schoots, K., \& Van der Zwaan, B. (2009). Use and limitations of learning curves for energy technology policy: A component-learning hypothesis. Energy policy, 37(7), 2525-2535.

Finch, B., \& Luebbe, R. (1995). The impact of learning rate and constraints on production line performance. The International Journal of Production Research 33(3), 631-642.

Flapper, S. D. P., Fransoo, J. C., Broekmeulen, R. A., \& Inderfurth, K. (2002). Planning and control of rework in the process industries: a review. Production Planning \& Control, 13(1), 26-34.

Franceschini, F., \& Galetto, M. (2002). Asymptotic defectiveness of manufacturing plants: An estimate based on process learning curves. International Journal of Production Research, 40(3), 537-545.

Frank, A. L. (2000). Injuries related to shiftwork. American Journal of Preventive Medicine, 18(4, Supplement 1), 33-36.

Freund, J., \& Takala, E.-P. (2001). A dynamic model of the forearm including fatigue. Journal of Biomechanics, 34(5), 597-605. 
Frey-Law, L. A., Looft, J. M., \& Heitsman, J. (2012). A three-compartment muscle fatigue model accurately predicts joint-specific maximum endurance times for sustained isometric tasks. Journal of Biomechanics, 45(10), 1803-1808.

Fritzsche, L., Wegge, J., Schmauder, M., Kliegel, M., \& Schmidt, K.-H. (2014). Good ergonomics and team diversity reduce absenteeism and errors in car manufacturing. Ergonomics(ahead-of-print), $1-14$.

Froehle, C. M., \& White, D. L. (2013). Interruption and Forgetting in Knowledge-Intensive Service Environments. Production and Operations Management.

Gander, P., Hartley, L., Powell, D., Cabon, P., Hitchcock, E., Mills, A., \& Popkin, S. (2011). Fatigue risk management: Organizational factors at the regulatory and industry/company level. Accident Analysis \& Prevention, 43(2), 573-590.

Gangopadhyay, S., \& Das, T. (2012). An ergonomic study on the onset of mental fatigue among the load handling workers of a central market area in Kolkata. Work, 41(SUPPL.1), 2467-2471.

Garvin, D. A. (2000). Learning in Action: A Guide to Putting the Learning Organization to Work. Boston, Massachusetts: Harvard Business School Press.

Geisinger, K. (2003). GAIN Guide to Methods \& Tools for Safety Analysis in Air Traffic Management. In A. T. R. M. Program (Ed.), (First ed., pp. 135): Airbus.

Gel, E. S., Hopp, W. J., \& Van Oyen, M. P. (2007). Hierarchical cross-training in work-in-processconstrained systems. IIE Transactions, 39(2), 125-143.

Giuntini, R. E. (2000). Mathematical characterization of human reliability for multi-task system operations. Paper presented at the 2000 IEEE International Conference on Systems, Man, and Cybernetics.

Globerson, S., \& Levin, N. (1987). Incorporating forgetting into learning curves. International Journal of Operations and Production Management, 7(4), 80-93.

Globerson, S., Levin, N., \& Shtub, A. (1989). Impact of breaks on forgetting when performing a repetitive task. IIE Transactions (Institute of Industrial Engineers), 21(4), 376-381.

Globerson, S., Nahumi, A., \& Ellis, S. (1998). Rate of forgetting for motor and cognitive tasks. International Journal of Cognitive Ergonomics, 2(3), 181-191.

Glock, C. H., \& Jaber, M. Y. (2013a). An economic production quantity (EPQ) model for a customerdominated supply chain with defective items, reworking and scrap. International Journal of Services and Operations Management, 14(2), 236-251.

Glock, C. H., \& Jaber, M. Y. (2013b). A multi-stage production-inventory model with learning and forgetting effects, rework and scrap. Computers \& Industrial Engineering, 64(2), 708-720.

Gold, B. (1981). Changing Perspectives on Size, Scale, and Returns: An Interpretive Survey. Journal of Economic Literature, 19(1), 5-33. 
Goode, J. H. (2003). Are pilots at risk of accidents due to fatigue? Journal of Safety Research, 34(3), 309313.

Grandjean, E. (1979). Fatigue in industry. British Journal of Industrial Medicine, 36(Journal Article), 175-186.

Granger, G., \& Jen-Gwo Chen, J. (1994). Assessing Human Error Rate Probabilities in a Repetetive Assembly Process. In F. Aghazadeh (Ed.), Advances in Industrial Ergonomics and Safety IV (pp. 135-139): Taylor \& Francis.

Griffith, C. D., \& Mahadevan, S. (2011). Inclusion of fatigue effects in human reliability analysis. Reliability Engineering \& System Safety, 96(11), 1437-1447.

Grosse, E. H., \& Glock, C. H. (2013). An experimental investigation of learning effects in order picking systems. Journal of Manufacturing Technology Management, 24(6), 850-872.

Gruber, H. (1992). The learning curve in the production of semiconductor memory chips. - Applied Economics, 24(8), 885-894.

Grubler, A., \& Nemet, G. F. (2014). Sources and Consequences of Knowledge Depreciation. Energy Technology Innovation, 133.

Guimarães, L. M., Anzanello, M. J., \& Renner, J. S. (2012). A learning curve-based method to implement multifunctional work teams in the Brazilian footwear sector. Applied Ergonomics, 43(3), 541547.

Gulledge, Jr., T, \& Womer, N. (1990). Learning curves and production functions: An integration. Engineering Costs and Production Economics, 20(Journal Article), 3-12.

Gulliksen, H. (1934). A Rational Equation of the Learning Curve Based on Thorndike's Law of Effect. The Journal of General Psychology, 11(2), 395-434.

Hannaman, G., Spurgin, A., \& Lukic, Y. (1984). Human cognitive reliability model for PRA analysis. Palo Alto CA: Electronic Power Research Institute.

Heimerl, C., \& Kolisch, R. (2010). Work assignment to and qualification of multi-skilled human resources under knowledge depreciation and company skill level targets. International Journal of Production Research, 48(13), 3759-3781.

Helmreich, R. L. (2000). On error management: lessons from aviation. BMJ: British Medical Journal, $320(7237), 781$.

Hewitt, D., Sprague, K., Yearout, R., Lisnerski, D., \& Sparks, C. (1992). The effects of unequal relearning rates on estimating forgetting parameters associated with performance curves. International Journal of Industrial Ergonomics, 10(3), 217-224.

Hoffman, T. R. (1968). Effect of prior experience on learning curve parameters. Journal of Industrial Engineering, 19(8), 412-413. 
Hollnagel, E. (1996). Reliability analysis and operator modelling. Reliability Engineering \& System Safety, 52(3), 327-337.

Hopp, W. J., \& Oyen, M. P. (2004). Agile workforce evaluation: a framework for cross-training and coordination. IIE Transactions, 36(10), 919-940.

Horton, L. M., Nussbaum, M. A., \& Agnew, M. J. (2012). Effects of rotation frequency and task order on localised muscle fatigue and performance during repetitive static shoulder exertions. Ergonomics, 55(10), 1205-1217.

Hottenstein, M. P., \& Bowman, S. A. (1998). Cross-training and worker flexibility: A review of DRC system research. Journal of High Technology Management Research, 9(2), 157-174.

Hovland, C. I. (1951). Human learning and retention. In S. S. Stevens (Ed.), Handbook of experimental psychology. (pp. 613-689). Oxford, England: Wiley.

Ihara, R. (2007). Toyota's assembly line: a view from the factory floor (H. Clarke, Trans.): Trans Pacific Press.

Inman, R. R., Jordan, W. C., \& Blumenfeld, D. E. (2004). Chained cross-training of assembly line workers. International Journal of Production Research, 42(10), 1899-1910.

Isaac, A., Shorrock, S. T., \& Kirwan, B. (2002). Human error in European air traffic management: the HERA project. Reliability Engineering \& System Safety, 75(2), 257-272.

Iwanaga, K., Saito, S., Shimomura, Y., Harada, H., \& Katsuura, T. (2000). The effect of mental loads on muscle tension, blood pressure and blink rate. Journal of Physiological Anthropology and Applied Human Science, 19(3), 135-141.

Jaber, M. Y. (2006). Learning and forgetting models and their applications Handbook of industrial and system engineering (pp. 1-22): Taylor and Francis Group, LLC.

Jaber, M. Y. (2014). Learning and forgetting models and their applications. In A. B. Badiru (Ed.), Handbook of industrial and system engineering (Second ed., pp. 535-566). FL: Baco Rotan: CRC Press.

Jaber, M. Y., \& Bonney, M. (1996). Production breaks and the learning curve: the forgetting phenomenon. Applied Mathematical Modelling, 20(2), 162-169.

Jaber, M. Y., \& Bonney, M. (1997). A comparative study of learning curves with forgetting. Applied Mathematical Modelling, 21(8), 523-531.

Jaber, M. Y., \& Bonney, M. (1999). The economic manufacture/order quantity (EMQ/EOQ) and the learning curve: past, present, and future. International Journal of Production Economics, 59(1), 93-102.

Jaber, M. Y., \& Bonney, M. (2003). Lot sizing with learning and forgetting in set-ups and in product quality. International Journal of Production Economics, 83(1), 95-111. 
Jaber, M. Y., \& Bonney, M. (2011). The lot sizing problem and the learning curve: A review. In M. Y. Jaber (Ed.), Learning Curves, Theory, Models and Applications (pp. 265-291): Boca Raton: CRC Press.

Jaber, M. Y., Bonney, M., \& Guiffrida, A. L. (2010). Coordinating a three-level supply chain with learning-based continuous improvement. International Journal of Production Economics, 127(1), $27-38$.

Jaber, M. Y., Bonney, M., \& Moualek, I. (2009). Lot sizing with learning, forgetting and entropy cost. International Journal of Production Economics, 118(1), 19-25.

Jaber, M. Y., \& Guiffrida, A. L. (2004). Learning curves for processes generating defects requiring reworks. European Journal of Operational Research, 159(3), 663-672.

Jaber, M. Y., \& Guiffrida, A. L. (2008). Learning curves for imperfect production processes with reworks and process restoration interruptions. European Journal of Operational Research, 189(1), 93-104.

Jaber, M. Y., \& Khan, M. (2010). Managing yield by lot splitting in a serial production line with learning, rework and scrap. International Journal of Production Economics, 124(1), 32-39.

Jaber, M. Y., \& Kher, H. V. (2002). The dual-phase learning-forgetting model. International Journal of Production Economics, 76(3), 229-242.

Jaber, M. Y., \& Kher, H. V. (2005). Workforce cross-training with learning in production and reworks. Paper presented at the 18th International Conference on Production Research-ICPR 18, Salerno, Italy.

Jaber, M. Y., Kher, H. V., \& Davis, D. J. (2003). Countering forgetting through training and deployment. International Journal of Production Economics, 85(1), 33-46.

Jaber, M. Y., \& Neumann, W. P. (2010). Modelling worker fatigue and recovery in dual-resource constrained systems. Computers and Industrial Engineering, 59(1), 75-84.

Jaber, M. Y., \& Sikström, S. (2004a). A note on "An empirical comparison of forgetting models". IEEE Transactions on Engineering Management, 51(2), 233-234.

Jaber, M. Y., \& Sikström, S. (2004b). A numerical comparison of three potential learning and forgetting models. International Journal of Production Economics, 92(3), 281-294.

Jahandideh, S. (2012). Job Scheduling considering both Mental Fatigue and Boredom. Master of Applied Science, University of Ottawa.

Jamal, A., Sarker, B. R., \& Mondal, S. (2004). Optimal manufacturing batch size with rework process at a single-stage production system. Computers \& Industrial Engineering, 47(1), 77-89.

James, D. M., \& William, S. M. (1993). Occupational risk factors associated with soft tissue disorders of the shoulder: a review of recent investigations in the literature. Ergonomics, 36(6), 697-717. 
Jansen, N. W. H., Kant, I., \& Van den Brandt, P. A. (2002). Need for recovery in the working population: Description and associations with fatigue and psychological distress. International Journal of Behavioral Medicine, 9(4), 322-340.

Jenab, K., Fang, L., \& Noori, K. R. (2009). Environmental performance of systems from reliability perspective. International Journal of Industrial and Systems Engineering, 4(4), 431-445.

Ji, Q., Lan, P., \& Looney, C. (2006). A probabilistic framework for modeling and real-time monitoring human fatigue. Systems, Man and Cybernetics, Part A: Systems and Humans, IEEE Transactions on, 36(5), 862-875.

Jordan, R. B. (1958). Learning how to use the learning curve. N.A.A. Bulletin, 39(5), 27-39.

Jorgensen, M., Davis, K., Kotowski, S., Aedla, P., \& Dunning, K. (2005). Characteristics of job rotation in the Midwest US manufacturing sector. Ergonomics, 48(15), 1721-1733.

Kang, K., \& Hur, I. (2013). Differential and Depreciation Effects of Shared Experience: Evidence from Software Projects. Paper presented at the System Sciences (HICSS), 2013 46th Hawaii International Conference on.

Karaoz, M., \& Albeni, M. (2005). Dynamic technological learning trends in Turkish manufacturing industries. Technological Forecasting and Social Change, 72(7), 866-885.

Kellogg, R. (2009). Learning by drilling: Inter-firm learning and relationship persistence in the texas oilpatch: National Bureau of Economic Research.

Kennedy, W. G., \& Trafton, J. G. (2007). Long-term symbolic learning. Cognitive Systems Research, $8(3), 237-247$.

Keyserling, W. M., \& Chaffin, D. B. (1986). Occupational ergonomics-methods to evaluate physical stress on the job. Annual Review of Public Health, 7(1), 77-104.

Khan, M., Jaber, M. Y., \& Plaza, M. (2011). Linking Quality to Learning- A Review. In M. Y. Jaber (Ed.), Learning Curves, Theory, Models and Applications (pp. 211-235): CRC Press, Taylor and Francis Group.

Kher, H. V. (2000). Examination of flexibility acquisition policies in dual resource constrained job shops with simultaneous worker learning and forgetting effects. The Journal of the Operational Research Society, 51(5), 592-601.

Kher, H. V., Malhotra, M. K., Philipoom, P. R., \& Fry, T. D. (1999). Modeling simultaneous worker learning and forgetting in dual resource constrained systems. European Journal of Operational Research, 115(1), 158-172.

Khouja, M. (2005). The use of minor setups within production cycles to improve product quality and yield. International Transactions in Operational Research, 12(4), 403-416.

Kim, I., \& Seo, H. L. (2009). Depreciation and transfer of knowledge: an empirical exploration of a shipbuilding process. International Journal of Production Research, 47(7), 1857-1876. 
Kim, S., \& Nembhard, D. A. (2010). Cross-trained staffing levels with heterogeneous learning/forgetting. Engineering Management, IEEE Transactions on, 57(4), 560-574.

Kirwan, B. (1992). Human error identification in human reliability assessment. Part 1: Overview of approaches. Applied Ergonomics, 23(5), 299-318.

Kleiner, M. M., Nickelsburg, J., \& Pilarski, A. M. (2011). Organizational and Individual learning and forgetting. Industrial and Labor Relations Review, 65(1), 68-81.

Konz, S. A. (1998). Work/Rest: Part II - The scientific basis (knowledge base) for the guide. International Journal of Industrial Ergonomics, 22(1-2), 73-99.

Konz, S. A., \& Johnson, S. (2000). Work design: industrial ergonomics: Holcomb Hathaway.

Koole, G., \& Pot, A. (2006). An overview of routing and staffing algorithms in multi-skill customer contact centers. Submitted for publication.

Kopardekar, P., \& Mital, A. (1994). The effect of different work-rest schedules on fatigue and performance of a simulated directory assistance operator's task. Ergonomics, 37(10), 1697-1707.

Kortge, G. D., Okonkwo, P. A., Burley, J. R., \& Kortge, J. D. (1994). Linking experience, product life cycle, and learning curves: Calculating the perceived value price range. Industrial Marketing Management, 23(3), 221-228.

Koulamas, C. (1992). Quality improvement through product redesign and the learning curve. Omega, 20(2), 161-168.

Krajcarski, S., \& Wells, R. (2008). The time variation pattern of mechanical exposure and the reporting of low back pain. Theoretical Issues in Ergonomics Science, 9(1), 45-71.

Kristal-Boneh, E., Froom, P., Harari, G., \& Ribak, J. (1996). Fatigue Among Israeli Industrial Employees. Journal of Occupational and Environmental Medicine, 38(11), 1145-1150.

Lapré, M. A., Mukherjee, A. S., \& Van Wassenhove, L. N. (2000). Behind the learning curve: Linking learning activities to waste reduction. Management Science, 46(5), 597-611.

Leung, A. W. S., Chan, C. C. H., Ng, J. J. M., \& Wong, P. C. C. (2006). Factors contributing to officers' fatigue in high-speed maritime craft operations. Applied Ergonomics, 37(5), 565-576.

Levin, D. Z. (2000). Organizational learning and the transfer of knowledge: An investigation of quality improvement. Organization Science, 11(6), 630-647.

Levitt, B., \& March, J. G. (1988). Organizational learning. Annual review of sociology, 319-340.

Liles, D. (1986). The application of the job severity index to job design for the control of manual materials-handling injury. Ergonomics, 29(1), 65-76.

Lindstrom, L., Kadefors, R., \& Petersen, I. (1977). An electromyographic index for localized muscle fatigue. Journal of applied physiology, 43(4), 750-754. 
Liu, J. Z., Brown, R. W., \& Yue, G. H. (2002). A Dynamical Model of Muscle Activation, Fatigue, and Recovery. Biophysical Journal, 82(5), 2344-2359.

Lobo, B. J., Wilson, J. R., Thoney, K. A., Hodgson, T. J., \& King, R. E. (2014). A practical method for evaluating worker-allocations in large-scale dual resource constrained job shops. IIE Transactions(just-accepted).

Lodree Jr, E. J., Geiger, C. D., \& Jiang, X. (2009). Taxonomy for integrating scheduling theory and human factors: Review and research opportunities. International Journal of Industrial Ergonomics, 39(1), 39-51.

Luczak, H., \& Mueller, T. (1994). Worker attention and fatigue. In G. Salvendy \& W. Karwowski (Eds.), Design of work and development of personnel in advanced manufacturing (pp. 463-492): John Wiley \& Sons, Inc.

Ma, L., Chablat, D., Bennis, F., \& Zhang, W. (2009). A new simple dynamic muscle fatigue model and its validation. International Journal of Industrial Ergonomics, 39(1), 211-220.

MacDonald, W. (2003). The impact of job demands and workload on stress and fatigue. Australian Psychologist, 38(2), 102-117.

MacLeod, M. D., \& Macrae, C. N. (2001). Gone But Not Forgotten: The Transient Nature of RetrievalInduced Forgetting. Psychological Science, 12(2), 148-152.

Mazur, J. E., \& Hastie, R. (1978). Learning as accumulation: A reexamination of the learning curve. Psychological Bulletin, 85(6), 1256-1274.

McCreery, J. K., \& Krajewski, L. J. (1999). Improving performance using workforce flexibility in an assembly environment with learning and forgetting effects. International Journal of Production Research, 37(9), 2031-2058.

McKenna, S. P., \& Glendon, A. I. (1985). Occupational first aid training: Decay in cardiopulmonary resuscitation (CPR) skills. Journal of Occupational Psychology, 58(2), 109-117.

Medbo, L. (2003). Assembly work execution and materials kit functionality in parallel flow assembly systems. International Journal of Industrial Ergonomics, 31(4), 263-281.

Meerding, W., IJzelenberg, W., Koopmanschap, M., Severens, J., \& Burdorf, A. (2005). Health problems lead to considerable productivity loss at work among workers with high physical load jobs. Journal of clinical epidemiology, 58(5), 517-523.

Mensik, G.-J., \& Raaijmakers, J. G. (1988). A model for interference and forgetting. Psychological Review, 95(4), 434-455.

Meyr, H. (2004). Supply chain planning in the German automotive industry. OR spectrum, 26(4), 447470.

Michalos, G., Makris, S., \& Chryssolouris, G. (2013). The effect of job rotation during assembly on the quality of final product. CIRP Journal of Manufacturing Science and Technology. 
Misawa, T., Yoshino, K., \& Shigeta, S. (1984). An experimental study on the duration of a single spell of work on VDT (visual display terminal) performance. Sangyo Igaku, 26(4), 296-302.

Mital, A., Bishu, R. R., \& Manjunath, S. G. (1991). Review and evaluation of techniques for determining fatigue allowances. International Journal of Industrial Ergonomics, 8(2), 165-178.

Mitchell, F. M. (2000). The scope and organization of production: Firm dynamics over the learning curve. The RAND Journal of Economics, 31(1), 180-205.

Molaoa, T. (2008). Managing of work stress among blue collar workers in the mining industry in Lesotho. University of Fort Hare.

Mosleh, A., \& Chang, Y. (2004). Model-based human reliability analysis: prospects and requirements. Reliability Engineering \& System Safety, 83(2), 241-253.

Muggleton, J., Allen, R., \& Chappell, P. (1999). Hand and arm injuries associated with repetitive manual work in industry: a review of disorders, risk factors and preventive measures. Ergonomics, 42(5), 714-739.

Murata, A., Uetake, A., \& Takasawa, Y. (2005). Evaluation of mental fatigue using feature parameter extracted from event-related potential. International Journal of Industrial Ergonomics, 35(8), 761-770.

Myszewski, J. M. (2010). Mathematical model of the occurrence of human error in manufacturing processes. Quality and Reliability Engineering International, 26(8), 845-851.

Myszewski, J. M. (2012). Management responsibility for human errors. The TQM Journal, 24(4), 326337.

Nembhard, D. A. (2000). The Effects of Task Complexity and Experience on Learning and Forgetting: A Field Study. Human Factors: The Journal of the Human Factors and Ergonomics Society, 42(2), 272-286.

Nembhard, D. A., \& Osothsilp, N. (2001). An empirical comparison of forgetting models. Engineering Management, IEEE Transactions on, 48(3), 283-291.

Nembhard, D. A., \& Uzumeri, M. V. (2000a). Experiential learning and forgetting for manual and cognitive tasks. International Journal of Industrial Ergonomics, 25(4), 315-326.

Nembhard, D. A., \& Uzumeri, M. V. (2000b). An individual-based description of learning within an organization. IEEE Transactions on Engineering Management, 47(3), 370-378.

Nembhard, H. B., Nembhard, D. A., \& Gurses, A. P. (2002). Real options modeling for valuing worker flexibility: Citeseer.

Niebel, B. W., Freivalds, A., \& Niebel, B. W. (1999). Methods, standards, and work design: WCB/McGraw-Hill Boston, MA. 
Noroozi, A., Khakzad, N., Khan, F., MacKinnon, S., \& Abbassi, R. (2013). The role of human error in risk analysis: application to pre and post-maintenance procedures of process facilities. Reliability Engineering \& System Safety.

Nozaki, S., Tanaka, M., Mizuno, K., Ataka, S., Mizuma, H., Tahara, T., Sugino, T., Shirai, T., Eguchi, A., Okuyama, K., Yoshida, K., Kajimoto, Y., Kuratsune, H., Kajimoto, O., \& Watanabe, Y. (2009). Mental and physical fatigue-related biochemical alterations. Nutrition, 25(1), 51-57.

O'Leonard, K. (2013). The Corporate Learning Factbook® 2013: Benchmarks, Trends and Analysis of the US Training Market. USA: Bersin \& Associates.

Omar, M., Mears, L., Kurfess, T., \& Kiggans, R. (2011). Organizational learning in automotive manufacturing: a strategic choice. Journal of Intelligent Manufacturing, 22(5), 709-715.

Othman, M., Gouw, G. J., \& Bhuiyan, N. (2012). Workforce scheduling: A new model incorporating human factors. Journal of Industrial Engineering \& Management, 5(2).

Pal, B., Sana, S. S., \& Chaudhuri, K. (2013). Maximising profits for an EPQ model with unreliable machine and rework of random defective items. International Journal of Systems Science, 44(3), 582-594.

Park, K. S., \& Jung, K. T. (1996). Considering performance shaping factors in situation-specific human error probabilities. International Journal of Industrial Ergonomics, 18(4), 325-331.

Park, P. S., \& Bobrowski, P. M. (1989). Job release and labor flexibility in a dual resource constrained job shop. Journal of Operations Management, 8(3), 230-249.

Perez, J. (2011). Virtual human factors tools for proactive ergonomics qualitative exploration and method developement. Master of Applied Science, Ryerson University.

Peter Chiu, Y.-S., Chen, K.-K., Cheng, F.-T., \& Wu, M.-F. (2010). Optimization of the finite production rate model with scrap, rework and stochastic machine breakdown. Computers \& mathematics with applications, 59(2), 919-932.

Peterson, R. T., \& Jun, M. (2007). The Plateau Syndrome: The Problem and Alternative Solutions. Journal of Selling \& Major Account Management, 7(2), 18-36.

Pinker, E. J., \& Shumsky, R. A. (2000). The efficiency-quality trade-off of cross-trained workers. Manufacturing \& Service Operations Management, 2(1), 32-48.

Porteus, E. L. (1986). Optimal Lot Sizing, Process Quality Improvement and Setup Cost Reduction. Operations Research, 34(1), 137-144.

Punnett, L., \& Wegman, D. H. (2004). Work-related musculoskeletal disorders: the epidemiologic evidence and the debate. Journal of Electromyography and Kinesiology, 14(1), 13-23.

Reason, J. (1990). Human error: Cambridge university press.

Reis, D. A. (1991). Learning curves in food services. The Journal of the Operational Research Society, 42(8), 623-629. 
Ricci, J. A., Chee, E., Lorandeau, A. L., \& Berger, J. (2007). Fatigue in the U.S. Workforce: Prevalence and Implications for Lost Productive Work Time. Journal of Occupational and Environmental Medicine, 49(1), 1-10

Robbins, T. R., Harrison, T. P., \& Medeiros, D. J. (2007). Partial cross training in call centers with uncertain arrivals and global service level agreements. Paper presented at the Simulation Conference, 2007 Winter.

Rohmert, W. (1973a). Problems in determining rest allowances: Part 1: Use of modern methods to evaluate stress and strain in static muscular work. Applied Ergonomics, 4(2), 91-95.

Rohmert, W. (1973b). Problems of determination of rest allowances Part 2: Determining rest allowances in different human tasks. Applied Ergonomics, 4(3), 158-162.

Rohmert, W. (1987). Physiological and psychological workload measurement and analysis Handbook of Human Factors: John Wiley \& Sons Inc.

Rose, L., Ericson, M., Glimskär, B., Nordgren, B., \& Örtengrend, R. (1992). Ergo-Index. A model to determine pause needs after fatigue and pain reactions during work. Paper presented at the Annual International Industrial Ergonomics and Safety Conference, Denver, Colorado, USA.

Roy, M. D., Sana, S. S., \& Chaudhuri, K. (2013). An economic production lot size model for defective items with stochastic demand, backlogging and rework. IMA Journal of Management Mathematics.

Saijo, Y., Ueno, T., \& Hashimoto, Y. (2008). Twenty-four-hour shift work, depressive symptoms, and job dissatisfaction among Japanese firefighters. American Journal of Industrial Medicine, 51(5), 380391.

Sana, S. S. (2010). An economic production lot size model in an imperfect production system. European Journal of Operational Research, 201(1), 158-170.

Schuster, M., \& Rhodes, S. (1985). The impact of overtime work on industrial accident rates. Industrial Relations: A Journal of Economy and Society, 24(2), 234-246.

Sebrina, \& Diawati, L. (2012, 2012). The impact of learning on assembly line capacity (A case study of an Indonesia car factory). Paper presented at the IEEE 6th International Conference on Management of Innovation and Technology, ICMIT 2012.

Serel, D. A., Dada, M., Moskowitz, H., \& Plante, R. D. (2003). Investing in quality under autonomous and induced learning. IIE Transactions, 35(6), 545-555.

Shafer, S. M., Nembhard, D. A., \& Uzumeri, M. V. (2001). The effects of worker learning, forgetting, and heterogeneity on assembly line productivity. Management Science, 47(12), 1639-1653.

Shahraki, S., \& Bin Abu Bakar, N. (2011). Review of relationship between knowledge of management, workforce productivity and nervous fatigue. African Journal of Business Management, 5(34), 13089-13094. 
Sherman, K. (2003). An Evaluation of Fatigue and Performance Changes During Intermittent Overhead Work. Master of Science, Virginia Polytechnic Institute and State University.

Sikström, S., \& Jaber, M. Y. (2002). The power integration diffusion model for production breaks. Journal of Experimental Psychology: Applied, 8(2), 118-126.

Sikström, S., \& Jaber, M. Y. (2012). The Depletion-Power-Integration-Latency (DPIL) model of spaced and massed repetition. Computers \& Industrial Engineering, 63(1), 323-337.

Sikström, S., Jaber, M. Y., \& Neumann, W. P. . (2011). Influence of breaks in learning on forgetting curves. In M. Y. Jaber (Ed.), Learning Curves: Theory, Models, and Applications (pp. 163-172). FL: Baco Raton: CRC Press.

Sjøgaard, G. (1985). Intramuscular changes during long-term contraction. Paper presented at the The ergonomics of working postures: models, methods and cases. Proceedings of the First International Occupational Ergonomics Symposium, Zadar, Yugoslavia.

Smith, D. J. (2011). Reliability, maintainability and risk: Practical safety-related systems engineering methods: Access Online via Elsevier.

Smith, L., Tanigawa, T., Takahashi, M., Mutou, K., Tachibana, N., Kage, Y., \& Iso, H. (2005). Shiftwork locus of control, situational and behavioural effects on sleepiness and fatigue in shiftworkers. Industrial health, 43(1), 151-170.

Sonnentag, S., \& Zijlstra, F. R. H. (2006). Job characteristics and off-job activities as predictors of need for recovery, well-being, and fatigue. Journal of Applied Psychology, 91(2), 330.

Stratman, J. K., Roth, A. V., \& Gilland, W. G. (2004). The deployment of temporary production workers in assembly operations: a case study of the hidden costs of learning and forgetting. Journal of Operations Management, 21(6), 689-707.

Subedi, D. K. (2006). A Resource Based View Of Quality: Managers' Role In Developing Quality As A Weapon Of Competitive Advantage. Journal of Business \& Economics Research (JBER), 4(6).

Swan, A., \& Guttman, H. (1983). A handbook of human reliability analysis with emphasis on nuclear power plant applications. USNRC-Nureg/CR-1278, US Nuclear Regulatory Commission, Washington DC.

Syverson, C. (2010). What determines productivity? : National Bureau of Economic Research.

Takahashi, N. (2013). Behind the Learning Curve. Annals of Business Administrative Science, 12(4), 167179.

Taleizadeh, A. A., Wee, H.-M., \& Jalali-Naini, S. G. (2013). Economic production quantity model with repair failure and limited capacity. Applied Mathematical Modelling, 37(5), 2765-2774.

Tang, A., Owen, C., Biocca, F., \& Mou, W. (2003). Comparative effectiveness of augmented reality in object assembly. Paper presented at the Proceedings of the SIGCHI Conference on Human Factors in Computing Systems. 
Teng, J. T., \& Thompson, G. L. (1996). Optimal strategies for general price - Quality decision models of new products with learning production costs. European Journal of Operational Research, 93(3), 476-489.

Tessitore, A., Meeusen, R., Cortis, C., \& Capranica, L. (2007). Effects of different recovery interventions on anaerobic performance following preseason soccer training. Journal of Strength and Conditioning Research, 21(3), 745.

Teyarachakul, S., Çömez, D., \& Tarakci, H. (2014). Steady-state skill levels of workers in learning and forgetting environments: A dynamical system analysis. European Journal of Operational Research, 232(1), 9-21.

Thannimalai, P., Kadhum, M. M., Feng, C. J., \& Ramadass, S. (2013). A glimpse of cross training models and workforce scheduling optimization. Paper presented at the Computers \& Informatics (ISCI), 2013 IEEE Symposium on.

Tharmmaphornphilas, W., Green, B., Carnahan, B. J., \& Norman, B. A. (2003). Applying mathematical modeling to create job rotation schedules for minimizing occupational noise exposure. Aiha Journal, 64(3), 401-405.

Theorell-Haglöw, J., Lindberg, E., \& Janson, C. (2006). What are the important risk factors for daytime sleepiness and fatigue in women. Sleep, 29(6), 751-757.

Tirkel, I. (2013). Yield Learning Curve Models in Semiconductor Manufacturing. IEEE Transaction on Semiconductor Manufacturing, 26(4), 564-571.

Tucker, P. (2003). The impact of rest breaks upon accident risk, fatigue and performance: A review. Work and Stress, 17(2), 123-137.

Vasdev, N., Kass-Iliyya, A., Patel, A., Bedford, G., O'Riordon, A., Johnson, M. I., Durkan, G. C., \& Soomro, N. A. (2012). Developing a laparoscopic radical prostatectomy service: Defining the learning curve. Journal of Endourology, 26(7), 903-910.

Vitório, D. M., Masculo, F. S., \& Melo, M. O. B. C. (2012). Analysis of mental workload of electrical power plant operators of control and operation centers. Work, 41(SUPPL.1), 2831-2839.

Waldman, J. D. Y., Steven A. (2011). Learning and thinking systems. Health care management review, 28(1), 41-54.

Wang, H., \& Hu, S. J. (2010). Manufacturing complexity in assembly systems with hybrid configurations and its impact on throughput. CIRP Annals - Manufacturing Technology, 59(1), 53-56.

Wexler, A. S., Ding, J., \& Binder-Macleod, S. A. (1997). A mathematical model that predicts skeletal muscle force. Biomedical Engineering, IEEE Transactions on, 44(5), 337-348.

Wickelgren, W. A. (1972). Trace resistance and the decay of long-term memory. Journal of mathematical psychology, 9(4), 418-455.

Williams, J. (1986). HEART-a proposed method for assessing and reducing human error. Paper presented at the 9th Advances in Reliability Technology Symposium. 
Winwood, P. C., Winefield, A. H., Dawson, D., \& Lushington, K. (2005). Development and Validation of a Scale to Measure Work-Related Fatigue and Recovery: The Occupational Fatigue Exhaustion/Recovery Scale (OFER). Journal of Occupational and Environmental Medicine, 47(6), 594-606

Wright, T. P. (1936). Factors affecting the cost of airplanes. Journal of Aeronautical Sciences, 3(4), 122128.

Xia, T., \& Frey Law, L. A. (2008). A theoretical approach for modeling peripheral muscle fatigue and recovery. Journal of Biomechanics, 41(14), 3046-3052.

Xin, Y., \& Fan-Sen, K. (2010). The gearbox factory assembly line quality accident complexity mensuration due to human errors. Paper presented at the Computer, Mechatronics, Control and Electronic Engineering (CMCE), 2010 International Conference on.

Yelle, L. E. (1979). The learning curves: historical review and comprehensive survey. Decision Sciences, 10(2), 302-328.

Yeow, P. H. P., \& Nath Sen, R. (2003). Quality, productivity, occupational health and safety and cost effectiveness of ergonomic improvements in the test workstations of an electronic factory. International Journal of Industrial Ergonomics, 32(3), 147-163.

Yoshitake, H. (1978). Three characteristic patterns of subjective fatigue symptoms. Ergonomics, 21(3), 231-233.

Yue, H. (2005). Worker flexibility in dual resource constrained (DRC) shops. University Library Groningen.

Zamiska, J. R., Jaber, M. Y., \& Kher, H. V. (2007). Worker deployment in dual resource constrained systems with a task-type factor. European Journal of Operational Research, 177(3), 1507-1519.

Zangwill, W. I., \& Kantor, P. B. (1998). Toward a theory of continuous improvement and the learning curve. Management Science, 44(7), 910-920. 